\title{
Development of a Photogrammetric Tool for \\ Cranial Deformation Analysis in Infants
}

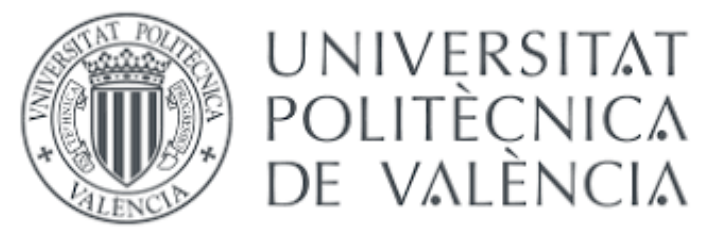

Inés Barbero García

Advisors:

José Luis Lerma García

Pablo Miranda Lloret

Photogrammetry and Laser Scanner Research Group (GiFLE)

Department of Cartographic Engineering Geodesy and Photogrammetry

Universitat Politècnica de València

September 2020 


\begin{abstract}
Image-based and range-based solutions have great potential for accurate measurements in many different fields, including medicine. Cranial deformation is a problem with high prevalence among infants during their first months of life. Cranial deformation has different causes and associated risks. Most common deformations have positional causes and have long been considered to cause aesthetical problems only. Nowadays, authors are pointing at these deformations as a possible indicator of future developmental delays. Another type of cranial deformation is caused by the early closing of cranial sutures, that can lead to important health risks and is usually corrected by surgery. Early detection of the deformation leads to better development prospects, as cranium is especially malleable during the first months of life. Good monitoring also provides better data for evaluation of the different correction procedures, improving the outcome of the patients over time. Cranial deformation evaluation methodologies can be divided into three mean groups: 1) Low-cost measurements carried out by simple tools such as calliper and measuring tape and even visual assessment; 2) Radiological tests (Computed Tomography (CT) and Magnetic Resonance Imaging (MRI)), considered the gold standard, but costly and highly invasive; and 3) Image-based and range-based commercial solutions, non-invasive but too costly to be implemented as part of the clinical routine in many hospitals and clinics.

In this doctoral thesis, the possibilities of photogrammetry as a method for cranial deformation assessment has been studied and a novel tool has been developed. The tool was required to be low-cost, objective and non-invasive, so no sedation would be required. The main challenge of the project was dealing with the movement of the infants during the consultation.
\end{abstract}


In the first place, single-lens reflex (SLR) cameras were used to obtain head models of the patients. The methodology provided the required accuracy but the data acquisition was time-consuming and required an important setup. The data processing was also time-consuming, being totally manual and requiring expert users. The necessities to automatically obtain accurate 3D models from moving infants were studied and a novel tool was developed. The developed tool consists of a coded cap that works together with a smartphone app to guide the user during the data acquisition. Therefore, no expertise is required for data acquisition. The data is later processed on a server and the model and deformation parameters are made available to the user in less than 4 minutes with an accuracy of $2 \mathrm{~mm}$. In order to make the model valuable for doctors, automatic measurements and deformation indexes are extracted from the model.

Photogrammetry is a suitable technique to obtain high quality data and overcome the limitations. Together with ad-hoc developed software, it is the base of a fully automatic tool that is optimised for its use in clinics and hospitals. The developed tool is considered highly cost-effective and an important improvement as it provides similar data as existing costly setups, but it is also low-cost and portable. 


\section{Resumen}

La fotogrametría ha demostrado un gran potencial para la obtención de mediciones precisas en diferentes campos de estudio, incluyendo la medicina. La deformación craneal es un problema con una alta prevalencia entre lactantes durante sus primeros años de vida, esta deformación puede tener diferentes causas y riesgos asociados. La causa más común de las deformaciones es posicional, este tipo de deformaciones se han asociado durante mucho tiempo a problemas únicamente estéticos. Sin embargo, diferentes autores han puesto de manifiesto la posible relación entre deformaciones posicionales y retrasos en el desarrollo. Otro tipo de deformación craneal es el causado por el cierre prematuro de algunas suturas craneales, que puede tener como consecuencia importantes riesgos para la salud de los lactantes y a menudo es corregido mediante cirugía. La detección precoz de estas deformaciones es importante para asegurar los mejores resultados futuros, ya que el cráneo es especialmente maleable durante los primeros meses de vida. La correcta monitorización de los pacientes también hace posible la evaluación de los diferentes tratamientos posibles y asegura una mejor evolución.

Las metodologías actuales para la evaluación de la deformación craneal pueden dividirse en tres grupos principales: 1) Mediciones de bajo coste, realizadas con dispositivos simples como cinta métrica y calibre, o mediante un simple análisis visual; 2) Pruebas radiológicas, en concreto Tomografía Computerizada e Imagen por Resonancia Magnética, son consideradas la opción más precisa pero tienen un alto coste y son invasivas y 3) Soluciones basadas en imagen o escáneres 3D, que no son invasivas pero si tienen un alto coste y, como consecuencia, no están presentes en la práctica clínica habitual. 
En esta tesis doctoral se han estudiado las posibilidades de la fotogrametría como método para evaluar la deformación craneal en lactantes y se ha desarrollado una herramienta para llevarlo a cabo. La herramienta presentada es de bajo coste, objetiva y no invasiva, ya que no es necesaria la sedación de los pacientes. El mayor reto de este proyecto fue la obtención de datos con lactantes en movimiento durante una consulta médica rutinaria.

En primer lugar, se utilizó cámara réflex para crear modelos 3D de la cabeza de los lactantes. La metodología generó modelos con una precisión adecuada pero la toma de datos requería largos periodos de tiempo y el montaje de diferentes dispositivos. El procesado de los datos también requería un largo periodo de tiempo al ser totalmente manual y requería de la intervención de un experto.

Se han estudiado las necesidades para el desarrollo de una herramienta capaz de obtener de forma automática modelos 3D de las cabezas de lactantes en movimiento, y se ha desarrollado una herramienta innovadora. Esta herramienta consiste en un gorro codificado, que funciona junto a una aplicación móvil y guía al usuario durante la toma de datos, como consecuencia, no es necesario ningún conocimiento de fotogrametría por parte de este. Los datos son procesados en un servidor, obteniéndose el modelo y los parámetros de deformación en un tiempo aproximado de 4 minutos y con una precisión de $2 \mathrm{~mm}$. Con el objetivo de hacer la herramienta útil para el personal médico, diferentes índices y parámetros de deformación se obtienen de forma automática a partir del modelo.

La fotogrametría es una técnica válida para obtener datos de gran calidad y superar los retos que supone esta aplicación en concreto. Junto con el software desarrollado, la fotogrametría es la base para de una herramienta totalmente automatizada y optimizada para su uso en clínicas y hospitales. La herramienta desarrollada es altamente coste-efectiva y mejora considerablemente las opciones disponibles, ya que permite precisiones similares a soluciones complejas, pero con la ventaja de ser totalmente portable y de bajo coste. 


\section{Resum}

La fotogrametria ha demostrat un gran potencial per a l'obtenció de mesuraments precises en diferents camps d'estudi, incloent la medicina. La deformació cranial és un problema amb una alta prevalença entre lactants durant els seus primers anys de vida, esta deformació pot tindre diferents causes i riscos associats. La causa més comuna de les deformacions és posicional, este tipus de deformacions s'han associat durant molt de temps a problemes únicament estètics. No obstant això, diferents autors han posat de manifest la possible relació entre deformacions posicionals i retards en el desenrotllament. Un altre tipus de deformació cranial és el causat pel tancament prematur d'algunes sutures cranials, que pot tindre com a conseqüència importants riscos per a la salut dels lactants i sovint és corregit per mitjà de cirurgia. La detecció precoç d'estes deformacions és important per a assegurar els millors resultats futurs ja que el crani és especialment mal $\cdot$ leable durant els primers mesos de vida. La correcta monitorització dels pacients també fa possible l'avaluació dels diferents tractaments possibles i assegura una millor evolució. Les metodologies actuals per a l'avaluació de la deformació cranial poden dividir-se en tres grups principals: 1) Mesuraments de baix cost, realitzades amb dispositius simples com a cinta mètrica i calibre, o per mitjà d'una simple anàlisi visual; 2) Proves radiològiques, en concret Tomografia Computeritzada i Imatge per Ressonància Magnètica, són considerades l'opció més precisa però tenen un alt cost i són invasives; i 3) Solucions basades en imatge o escàners 3D, que no són invasives però si tenen un alt cost i, com a conseqüència, no estan presents en la pràctica clínica habitual. En esta tesi doctoral s'han estudiat les possibilitats de la fotogrametria com a mètode per a avaluar la deformació cranial en lactants i s'ha desenrotllat una ferramenta per a dur-ho a terme. La ferramenta presentada és de baix cost, objectiva i no invasiva, ja que no és necessària 
la sedació dels pacients. El major repte d'este projecte va ser l'obtenció de dades amb lactants en moviment durant una consulta mèdica rutinària. En primer lloc, es va utilitzar càmera rèflex per a crear models $3 \mathrm{D}$ del cap dels lactants. La metodologia va generar models amb una precisió adequada però la presa de dades requeria llargs períodes de temps i el muntatge de diferents dispositius. El processat de les dades també requeria un llarg període de temps al ser totalment manual i requeria de la intervenció d'un expert. S'han estudiat les necessitats per al desenrotllament d'una ferramenta capaç d'obtindre de forma automàtica models 3D dels caps de lactants en moviment, i s'ha desenrotllat una ferramenta innovadora. Esta ferramenta consisteix en una capelina codificada, que funciona junt amb una aplicació mòbil i guia l'usuari durant la presa de dades, com a conseqüència, no cal cap coneixement de fotogrametria per part d'este. Les dades són processats en un servidor, obtenint-se el model i els paràmetres de deformació en un temps aproximat de 4 minuts i amb una precisió de 2 $\mathrm{mm}$. Amb l'objectiu de fer la ferramenta útil per al personal metge, diferents índexs i paràmetres de deformació s'obtenen de forma automàtica a partir del model. La fotogrametria és una tècnica vàlida per a obtindre dades de gran qualitat i superar els reptes que suposa esta aplicació en concret. Junt amb el programari desenrotllat, la fotogrametria és la base para d'una ferramenta totalment automatitzada i optimitzada per al seu ús en clíniques i hospitals. La ferramenta desenrotllada és altament cost-efectiva i millora considerablement les opcions disponibles, ja que permet precisions semblants a solucions complexes, però amb l'avantatge de ser totalment portable i de baix cost. 


\section{Acknowledgements}

I would like to thank all the people who have taken part in this project during the last four years. Firstly, my supervisors José Luis Lerma, from Universitat Politècnica de València and Pablo Miranda, Head of Pediatric Division of Neurosurgery at Hospital Universitari i Politècnic La Fe.

I want to mention all my colleagues from GiFLE at UPV, who have make the best of these years at the university. Berta Carrion and Silvia Blanco who dedicated entire months to work in the development of the tool. Miriam Cabrelles for her contribution in the photogrammetric part, Adolfo Molada, who has always been around, but joined the team during the last months and has been working to improve the solution since then, and Gabriel Ruitort. Together with the GiFLE team I want to include Ángel Marqués, for thinking of me for this project, and Gaspar Mora, for his development of the web and server. Also the R\&D and Innovation Management Office team at UPV for helping us taking this project to the real world.

From Hospital Universitari i Politècnic La Fe, the second home of this project, I would like to thank the whole neurosurgery team: Pablo Miranda, Giovanni Pancucci, Estela Plaza and the rest of their team. And also Pilar Garrido, who took part in the project way beyond the requirements of her job, and Maria, who has always found time in the complicated hospital schedules to help me.

Finally, I want to thank my family and friends for their support during this time. 
This project was founded by a predoctoral fellowship from Generalitat Valenciana and the European Social Fund, grant number ACIF/2017/056. 


\section{Contents}

List of Figures vii

List of Tables

Glossary xii

1 Introduction 1

1.1 Cranial deformation ..................... 1

1.1.1 Current methodologies for deformation measurement . . . . . . 2

1.2 Image-based and range-based solutions in medicine . . . . . . . . . 4

1.3 The development of the tool . . . . . . . . . . . 4

$1.3 .1 \quad$ Objectives . . . . . . . . . . . . . . . . . 4

1.3.2 The development process $\ldots \ldots \ldots \ldots$

1.3.2.1 The first tests . . . . . . . . . . . 5

1.3.2.2 A smartphone approach . . . . . . . . . . . 6

1.3.2.3 A marker-based approach . . . . . . . . . . 6 6

1.3.2.4 The app and web-based tool ......... 6 6

1.3.2.5 Evaluation of the deformation . . . . . . . . 7

1.4 Structure of the document . . . . . . . . . . . . . 7

2 A manual solution for the creation of head 3D models 9

2.1 Introduction . . . . . . . . . . . . . . . . 9

2.2 Materials and methods . . . . . . . . . . . . . . . . 10

2.2 .1 Planning . . . . . . . . . . . . . . 10

2.2 .2 Setup . . . . . . . . . . . . . . . 12

2.2 .3 Image acquisition . . . . . . . . . . . . . . . . . . 12 
2.2.4 Camera orientation and calibration . . . . . . . . . 14

2.2 .5 Creation of the 3D model . . . . . . . . . . . . 16

2.2 .6 Extraction of clinical measurements . . . . . . . . . 18

2.2 .7 Ellipsoid fitting . . . . . . . . . . . . . . 18

2.3 Results. . . . . . . . . . . . . . . . . . . . 19

2.3 .1 Visualization . . . . . . . . . . . . . . . . . 21

2.4 Discussion . . . . . . . . . . . . . . . . . . . . . . . . . . . 21

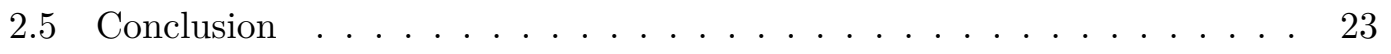

3 An smartphone-based application 25

3.1 Introduction . . . . . . . . . . . . . . . . . . . . . . . . 25

3.2 Methodology . . . . . . . . . . . . . . . . . . . . [26

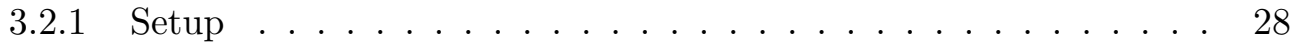

$3.2 .2 \quad$ Data acquisition $\ldots \ldots \ldots \ldots \ldots \ldots$

3.2 .3 Processing . . . . . . . . . . . . . . . . . . . 29

3.2 .4 Self-calibration . . . . . . . . . . . . . . 31

3.3 Results . . . . . . . . . . . . . . . . . . . . 33

3.4 Discussion . . . . . . . . . . . . . . . . . . 36

3.5 Conclusion . . . . . . . . . . . . . . . . . . 40

4 Adaptation to the best photogrammetric solution 41

4.1 Introduction . . . . . . . . . . . . . . . . . . 41

4.2 Methodology . . . . . . . . . . . . . . . . . . 44

4.2 .1 Calibration . . . . . . . . . . . . . . . . 44

4.2 .2 Three-dimensional model setup . . . . . . . . . . . . . 45

4.2 .3 Reference data . . . . . . . . . . . . . . . . . . 47

4.2 .4 Image acquisition . . . . . . . . . . . . . . . . . . 48

4.2 .5 Network design . . . . . . . . . . . . . . . . . . . . 48

4.2 .6 Model creation . . . . . . . . . . . . . . . . . 50 50

4.2 .7 Assessment . . . . . . . . . . . . . . . . . . . . . 50

4.3 Results . . . . . . . . . . . . . . . . . . 51

4.3 .1 Calibration . . . . . . . . . . . . . . 51

4.3.2 Three-dimensional models . . . . . . . . . . . . . 52

4.4 Discussion . . . . . . . . . . . . . . . . . . . . 555 
4.5 Conclusion . . . . . . . . . . . . . . . . . . . 559

5 Validation of the semiautomatic tool 61

5.1 Introduction . . . . . . . . . . . . . . . . . . . . . . . 61 61

5.2 Methodology ..................... 63

5.2 .1 Radiological data processing . . . . . . . . . . . 63

5.2 .2 Photogrammetry . . . . . . . . . . . . . . . 6 65

5.2 .3 Processing . . . . . . . . . . . . . . . 66

5.2 .4 Comparison . . . . . . . . . . . . . . . . 67

5.3 Results . . . . . . . . . . . . . . . . . 68

5.4 Discussion . . . . . . . . . . . . . . . . 73

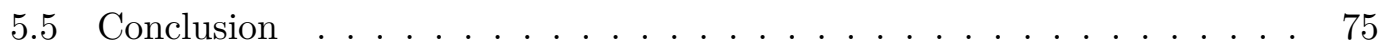

6 Registration of the models $\quad \mathbf{7 6}$

6.1 Introduction . . . . . . . . . . . . . . . . . 76

6.2 Methodology . . . . . . . . . . . . . . . . 77

6.2 .1 Data acquisition . . . . . . . . . . . . . . 777

6.2 .2 Registration . . . . . . . . . . . . . . . 77

6.2.2.1 PCA plus two tie points . . . . . . . . 77

6.2.2.2 PCA plus three tie points . . . . . . . . 77

6.2.2.3 Distance calculation . . . . . . . . . . . . 78

6.3 Results . . . . . . . . . . . . . . . . . . . 78

6.4 Discussion . . . . . . . . . . . . . . . . 79

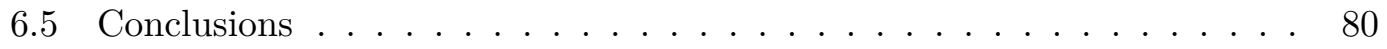

7 The automatic tool 81

7.1 Introduction . . . . . . . . . . . . . . . . . . . . 81

7.2 Materials and methods . . . . . . . . . . . . . . 83

7.2 .1 Automatic PhotoMeDAS tool . . . . . . . . . . . . . 83

7.2.1.1 Coded cap . . . . . . . . . . . . 83

7.2.1.2 Model creation . . . . . . . . . . . . . . . 855

7.2.1.3 Automatically-derived anthropometric linear magnitudes 87

7.2 .2 Validation methodology . . . . . . . . . . . . . . 88

7.2.2.1 Comparison between 3D models . . . . . . . . 89 
7.3 Results. . . . . . . . . . . . . . . . . . 90

7.4 Discussion . . . . . . . . . . . . . . . . . . 93

7.4 .1 The automatic tool .................. 93

7.4 .2 3D models . . . . . . . . . . . . . . . . 95

7.4.3 Automatically-derived anthropometric linear magnitudes . . . . 96

7.4.4 Comparison with current solutions . . . . . . . . . . . 98

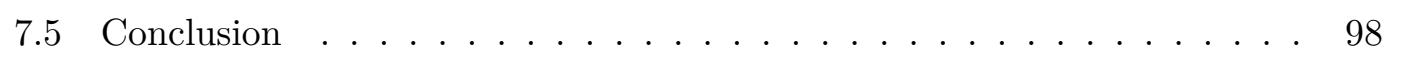

8 Overall Discussion $\quad 100$

9 Conclusions $\quad 103$

References 104 


\section{List of Figures}

1.1 The evolution of the cap $\ldots \ldots \ldots \ldots \ldots$

2.1 Methodology workflow . . . . . . . . . . . . . . . . 11

2.2 Sample of images used for 3D modelling. . . . . . . . . . . . 13

2.3 Oriented images (blue), with principal distances in 3D space (black lines) and sparse 3D point cloud. . . . . . . . . . . . . . 14

2.4 Camera distortion grid . . . . . . . . . . . . . . 16

2.5 Model creation steps . . . . . . . . . . . . . . . 17

2.6 Textured mesh. . . . . . . . . . . . . . . . . . . . . . . . 17

2.7 Diagram of reference for clinical measurements. . . . . . . . . . . 18

2.8 Marks placed on the patient cap . . . . . . . . . . . . 20

2.9 Ellipsoid fitting differences . . . . . . . . . . . . . . . . . . . . . . 22

3.1 Flow diagram. . . . . . . . . . . . . . . . . . . . 27

3.2 Cameras used during the image acquisition . . . . . . . . . . 29

3.3 Sample of images used for the processing and 3D modelling . . . . . . 30

3.4 3D models created using both cameras: (a) right and (b) left views, Canon EOS 1100D ; (c) right and (d) left views, smartphone video. . . . 32

3.5 Distortion grids . . . . . . . . . . . . . . . . . . . 35

3.6 Model showing the differences between the models as hypsometric colours. 37

4.1 Methodology workflow for calibration and 3D assessment . . . . . 45

4.2 Setup used for camera calibration that includes three orthogonal calibration panels, the sphere to be modelled and a calibrated ruler. Additional coded targets were incorporated to strengthen the geometry. . . . . . . 46

4.3 Three-dimensional model setup . . . . . . . . . . . . . . 4 47 


\section{LIST OF FIGURES}

4.4 Camera network geometry. . . . . . . . . . . . . . . 5 50

4.5 Examples of: (a) a complete three-dimensional model; (b), incomplete model; and (c) a model with small imperfections (bare) . . . . . . . . 51

4.6 Calibration distortion patterns for (a) the Samsung Galaxy S7 and (b) the Samsung Galaxy Trend. . . . . . . . . . . . . . . . . . 53

4.7 Box-and-whisker plots showing the accuracy of the models (using the mean error for each one $) \ldots \ldots \ldots \ldots \ldots$

4.8 Accuracy (top) and reliability (bottom) for the different network geometries based on the number of images, with and without targets. . . . . 557

5.1 Flow diagram used to assess the differences between 3D models. . . . . . 64

5.2 Image used for the creation of the 3D model. . . . . . . . . . . . . . 66

5.3 Calibration distortion pattern for Samsung Galaxy S5 (Scale: 2x). . . . 67

5.4 Mean distance differences and their standard deviations in relation to their patients' age. . . . . . . . . . . . . . . . . 69

5.5 Photogrammetry vs radiological test box-and-whisker plots showing the mean distance and the standard deviation results: a) all patients, b) under two years of age, c) older than two years, d) CT, and e) MRI. . . 70

5.6 Derived models for the 3rd patient: a, b) CT; c, d) Difference distances in the $3 \mathrm{D}$ model between the photogrammetric solution and the registered $\mathrm{CT}$.

5.7 Examples of errors found in MRI 3D models due to holes (a) and lying position of the patient during the data acquisition (c). These errors affect the distance differences between photogrammetry and MRI (b, d), respectively. . . . . . . . . . . . . . . . . . .

6.1 Top (a) and lateral (b) view of a model registered using CPA plus three tie points. Distances between two models (c) . . . . . . . . . . . 78

7.1 Implemented photogrammetric workflow. . . . . . . . . . . . . 83

7.2 a) Coded cap and stickers; b) view of the cap on the app. . . . . . . . . 84

7.3 PhotoMeDAS calculation. . . . . . . . . . . . . . 86

7.4 3D point cloud of the markers (a, side view; b, top view) and mesh (c, side view; d, top view) . . . . . . . . . . . . . . . . . . 87 
7.5 The head's local coordinate system. . . . . . . . . . 88

7.6 Targets. . . . . . . . . . . . . . . . . . . . . . . . . 889

7.7 Diagram displaying the distance computed between 3D models. . . . . . 90

7.8 Box plots of absolute differences between models for each test. . . . . . 91

7.9 Box plot of absolute differences between models for each test and model. 91

7.10 Box plot of signed differences for the accuracy tests. . . . . . . . . . 92

7.11 Mean and standard deviation for each created model. . . . . . . . . . 92

7.12 Representation of distances for Target 1. For the repeatability tests, the distances are shown on a reference sphere with its centre in the model centroid. For the accuracy tests, the distances are shown on the ground truth model. Histograms for each test are shown at the bottom (values in $\mathrm{mm}) \ldots \ldots \ldots \ldots \ldots \ldots \ldots$. . . . . . . . . . . . . . . . . . .

7.13 Comparison of perimeter measurements between two models of the same Target. . . . . . . . . . . . . . . . . . . 97 


\section{List of Tables}

2.1 Calibration Parameters from PhotoScan. Values are in mm. PW, pixel width; PH, pixel height; F, focal; XP and YP, principal point coordinates; K1, K2, K3 and K4, radial distortion coefficients; P1, P2, P3 and $\mathrm{P} 4$, tangential distortion coefficients. . . . . . . . . . 15

2.2 Clinical Measures Taken with Calipers and 3D Models. Values in mm. . 19

2.3 Distances between cranial model and the ellipsoid compared to global asymmetry. . . . . . . . . . . . . . . . 20

2.4 Clinical measurements for patients two to five. *Measures with points marked on the cap. . . . . . . . . . . . . . . . . . . . 21

3.1 Sensor specifications. . . . . . . . . . . . . . . 29

3.2 Additional calibration parameters for the two cameras (f, x0, y0 in mm), standard deviations and significance percentage according to Student's t-test. . . . . . . . . . . . . . . . . 34

3.3 Differences in estimated precision for both cameras. . . . . . . . 36

3.4 Fitted ellipsoid parameters for both models. . . . . . . . . . . . 36

4.1 Camera parameters. The mode actually used is highlighted in bold. . . 48

4.2 Additional calibration parameters and standard deviation $(\sigma)$ obtained using FOTOGIFLE. . . . . . . . . . . . . . . . . 52

4.3 Accuracy of the models. Mean and 65th percentile (P65) of the differences in distance. Incomplete models shown in yellow. Bare models with small imperfections in blue. Complete models in green. Models not achieved after the automatic photogrammetric workflow in orange. . . . 54 
5.1 Patients' information and distance difference parameters between radiological and photogrammetric models (differences in $\mathrm{mm}$ ). . . . . . . 68

6.1 Distances between registered models $(\mathrm{mm}) . \ldots \ldots$. . . . . . . 78

7.1 Mean and standard deviation (SD), Student's t-test 95\% confidence interval (95 CI), P-value (P) for comparison of the means between intrauser and interuser values, and $\mathrm{P}$ value for the hypothesis of an absolute true difference in mean $<1 \mathrm{~mm}(\mathrm{P} 1)$ (Values in $\mathrm{mm})$. . . . . . . . . . . 94 


\section{Glossary}

CT Computed Tomography

DICOM Digital Imaging and Communications in Medicine

DP Deformational Plagiocephaly

GRS Ground Reference System

ICP Iterative Closest Point

MRI Magnetic Resonance Imaging

PCA Principal Components Analysis

PLY Polygon File Format

RMSE Root Mean Square Error

SfM Structure from Motion

SIDS Sudden Infant Death Syndrome

SIFT Scale-Invariant Feature Transform

SLR Single-Lens Reflex

SURF Speeded-Up Robust Features 


\section{Introduction}

\subsection{Cranial deformation}

During infancy, the skull has to adapt to accommodate a growing brain. For this reason, during an early age, cranial bones are malleable and cranial sutures are open. Therefore, the infant's head is highly susceptible to be affected by deformation. Worth noticing is that cranial deformation is one of the most common problems treated by paediatric neurosurgeons (1). In most cases, the deformation is due to positional causes, but it can also be a consequence of early closure of cranial sutures. In the following paragraphs, we provide a brief and simplified explanation of the most common causes and types of cranial deformation.

The type of deformation with the highest incidence is positional plagiocephaly or deformational plagiocephaly (DP). According to different authors, if affects, at different degrees, between approximately 40-50\% of infants during the firsts weeks of life (2, 3, 4). DP is characterised by the flattening of an area of the head, resulting in asymmetry, typically occipital flattening associated with a compensatory ipsilateral frontal bosing. Flattening can also affect the posterior area of the head, not resulting in head asymmetry. This type of deformation is known as positional brachycephaly. DP and positional brachycephaly are caused by mechanical forces applied in utero, at birth or after birth, in most cases due to a maintained supine sleep position (5).

The incidence of positional plagiocephaly and positional brachycephaly experimented a dramatic increment in the 1990s as a consequence of the "Back to Sleep" Campaign of the American Association of Pediatrics. The campaign encouraged par- 


\section{INTRODUCTION}

ents to maintain infants on their backs while sleeping in order to prevent the sudden infant death syndrome (SIDS) (6). The initiative reduced SIDS by $50 \%$ (3) in the US and was adopted by many other countries (6) with positive results. This campaign had as consequence a decrease in prone sleep position from $70 \%$ to $24 \%$ from 1992 to 1996 (1). Before 1991, only $0.3 \%$ of children had been diagnosed with DP. It has been pointed out that the rise in DP after the "Back to Sleep" campaign may have motivated paediatricians to give cranial deformation higher importance and diagnose children with DP at younger ages (3).

DP and brachycephaly were considered by many experts to cause aesthetic problems only. However, different authors have found a correlation between DP and low developmental outcomes. It is not stated that DP is the cause for developmental problems, but it is to be considered a factor of developmental risk by some authors (7, 8). According to these results, the detection and evaluation of positional deformation at early ages should be considered extremely useful $(\underline{9}, 10)$. The usual treatment for DP and brachycephaly depends on the degree of the deformation. Mild cases are usually treated by repositioning and physical therapy, while more severe cases are often recommended to use orthotic helmets (3, 11).

A much less common deformation cause is craniosynostosis. Craniosynostosis is defined as a premature closing of one or various cranial sutures. The early closure leads to the restriction of growth perpendicular to the closed suture and the compensatory overgrowth perpendicular to the other, non-fused, sutures (12). The incidence of craniosynostosis is approximately 3.1 to 7.2 in 10.000 living births (13, 14). The early detection of craniosynostosis is extremely important as it can involve aesthetic problems but also visual deficit, hydrocephalus and increased cranial pressure (15). Single suture synostosis can be, starting for the most common, classified as follows: sagittal, coronal, metopical or lamboidal (12). Most craniosynostosis cases require surgery to correct the skull shape (3, 11).

\subsubsection{Current methodologies for deformation measurement}

Despite the importance and high prevalence of cranial deformation, there is not a widely used standard for its measurement and diagnosis. The measurement and assessment of the head shape is the only possible way to detect positional deformations as there are no inner causes that can be detected. For craniosynostosis, information of the bone 


\subsection{Cranial deformation}

sutures is required for diagnosis. However, the assessment of the head shape is the first step to find susceptible cases.

Many different approaches are followed for the detection and measurement of cranial deformation. Visual assessment is the most simple methodology for detection of cranial deformation. For visual evaluation, most paediatricians follow the indications given by Argenta (16). The most common approach for deformation assessment is the use of callipers and measuring tapes (17, 18). These devices allow the acquisition of a low number of measurements. The approach is low-cost and non-invasive. Normative guidelines for these manual measurements were given by Farkas (19). Authors have come to different conclusions on the reliability and accuracy of these measurements, with some of them estimating an intrauser accuracy of $2 \mathrm{~mm}(17,20)$. This methodology provides very limited information compared to a $3 \mathrm{D}$ model and the time required to acquire repeated measurements, in order to obtain acceptable accuracy, is high. The experts carrying out the measurement also require important training (21). The gold standards for the acquisition of medical 3D models are radiological tests, such as Computed Tomography (CT) and Magnetic Resonance Imaging (MRI). The accuracy of the 3D models obtained with these techniques are considered to have submilimeter accuracy. However, the accuracy of mesasurements manually taken on the models is set to approximately $2 \mathrm{~mm}(22)$. These methodologies have the unrivalled advantage of providing detailed information on the inner tissues. Information on the state of the bone sutures is required for accurate diagnosis of craniosynostosis. Despite their great advantages, these techniques are costly and, more importantly, highly invasive, as infants usually require sedation to stay still during the test. Moreover, CT implies radiation. For these reasons, these methodologies are not included in the routine tests and are only used when craniosynostosis is suspected (16). Moreover, some authors suggest that the lying position during these test can alter the position of soft tissues. As a consequence, it can be suggested that $\mathrm{CT}$ and MRI would not be the optimal way to evaluate the aesthetic outcomes (23, 24).

Image-based and range-based setups are able to provide complete information on head shape with high accuracy (25). There are many available devices with different characteristics. Some of the most widely known devices are 3dMD Head System (3dMD, Atlanta, GA) and STARScanner (Orthomerica, Orlando, FL). These are setups of several cameras capable of carrying out the necessary data acquisition in a very 


\section{INTRODUCTION}

short time and even with moving infants. They provide accurate 3D models of the external head's shape with submillimeter accuracy (25). These methodologies are used for different applications such as diagnosis (26), evaluation of surgical outcomes (27), definition of normal head parameters (28) or creation of orthotic helmets (29). The main disadvantages of these devices are their high cost and the lack of portability. For these reasons, they are not included as part of the clinical routine (4).

\subsection{Image-based and range-based solutions in medicine}

Photogrammetry has been proved to be an extremely useful tool for medical imaging. Different photogrammetric tools had been incorporated as part of the clinical practice, improving and providing higher accuracy than previously existing tools (30, 31). Image-based and range-based solutions are commonly used for documentation of the external body (32, 33). These technologies have a wide range of applications, such as surgery planning (34), deformation assessment (35), prosthetics design (36, 37, 38) or obtainment of anthropometric and craniofacial measurements for different purposes (39, 40, 41).

As happens with cranial deformation analysis technologies, the use of image-based and range-based solution in medicine needs to evolve to more portable and low-cost solutions in order to become part of the clinical routine (18, 42, 43).

Nowadays, advances in 3D technology and improvement in the quality of consumergrade cameras and computational capabilities of commonly used devices, make it possible to create accurate 3D models using non-specific technology, such as smartphones. This advance has open the possibility to develop methodologies that require a very low investment and can provide low-cost solutions for specific problems (44), even competing, in some specific problems, with the gold standards in the obtainment of 3D models in medicine, CT and MRI.

\subsection{The development of the tool}

\subsubsection{Objectives}

The project responded to the necessity of achieving accurate and objective ways to measure the cranial deformation of the infants during the usual consultation at hos- 
pitals, clinics or primary care units. The methodology should provide the possibility to carry out as many tests as necessary for detailed monitoring of the patients. The following objectives were identified:

- The tool should accurately represent the deformation. The results should provide more complete information that commonly used methodology using callipers and metric tape. A 3D model of the head would be ideal.

- The methodology should non-invasive, no sedation of the infants should be required. For this reason, the tool should not be affected by the infant's quick movements.

- The data acquisition should be quick. This would make the process more friendly for the infants and would also allow doctors to carry out the process during the limited time of the consultation.

- The tool should be low-cost and require the minimum possible setup so it can be used in different environments easily.

- The tool must be fully automatic. No knowledge of photogrammetry should be required from the users.

\subsubsection{The development process}

\subsubsection{The first tests}

The first step of the development, implemented under different research projects, was a proof of concept. The goal at this point was proving whether photogrammetry would be a valuable tool for the obtainment of accurate 3D models of infant's heads. We were aware that the movement of the infants would be a major problem during the acquisition of the data.

We visited the Hospital Universitari La Fe (in Valencia) to carry out the first tests. The imaging sensor on that occasion was a SLR high-end camera. We used tripods and took special care of the light conditions. A simple cap was fitted on every patient's head to avoid the effect of hair (Fig. 1.1 a). Many images were taken for each patient, always overestimating the requirements of a regular 3D modelling process. The results of this first test were limited. For most patients, the majority of the images were blurred due to 


\section{INTRODUCTION}

the infant's movement. After careful selection, the still images were masked to avoid the background and a 3D model of the patient's head was obtained. The data acquisition and processing were very time consuming and it was clear that another approach was required to create a clinically useful tool. At this point, some tests were also carried out using a range-based sensor, specifically a Sense 3D scanner (3D Systems, NC, USA). The methodology was quickly discarded as the infant's movement made impossible the obtainment of acceptable results.

\subsubsection{A smartphone approach}

The necessity to simplify the solution and deal with movement at the same time lead us to the use of smartphones. The slow-motion video mode would allow the acquisition of still images of moving infants. The data acquisition was simplified. No extra equipment, such as tripods, was required and the acquisition time was considerably lower. A specialist was still required to record the video, as all areas needed to be covered from different angles. The processing of the data was still difficult and time-consuming at this point. The process included the selection of useful video frames, the manual masking for each frame and, in most cases, the manual selection of tie-points between images, as the automatic solution would not provide good quality models.

\subsubsection{A marker-based approach}

Using easily identifiable markers on the cap greatly simplified the 3D modelling process. At the beginning, a small number of stickers were placed on the cap once it was on the patient's head. The stickers were not coded and were manually identified in the postprocessing step (Fig. 1.1p). After several tests, it became clear that a high quantity of automatically detected markers could greatly simplify 3D modelling (Fig. 1.1 k). To avoid increasing the time required for the data acquisition the markers were added to the cap beforehand and a new cap was developed (Fig. 1.1 d). It soon became clear that a high number of well-distributed markers would be enough for an accurate representation of the head and, therefore, images were no longer necessary.

\subsubsection{The app and web-based tool}

The automation of the 3D modelling process became possible with the use of coded targets. However, the acquisition of the data was still complicated and an incomplete 
set of data would easily hamper the creation of the 3D model. A smartphone application was developed to guide users during data acquisition. The smartphone would detect the markers and guide the user to assure the whole head was registered before sending the data to a server. Once in the server, the data was automatically processed to obtain the 3D model of the head. A new version of the cap was developed. It had a higher number of automatically-detected markers and it was optimised to facilitate its production (Fig 1.1p).

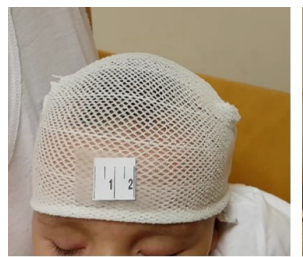

(a)

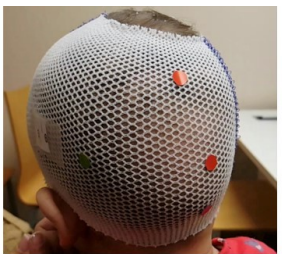

(b)

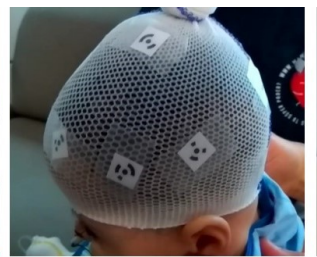

(c)

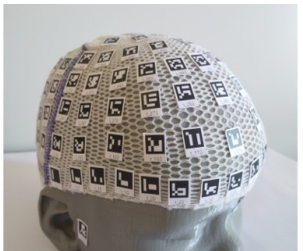

(d)

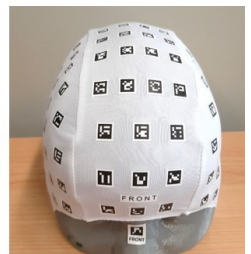

(e)

Figure 1.1: The evolution of the cap: (a) First version, a mesh with measuring tape for scale. (b)Including non-coded markers identifying cranial landmarks. (c) Some coded markers that are placed after placing the cap. (d) A high number of coded markers, whose relative position is known. (e) Last version of the cap. More and better-distributed markers. Optimized for production.

\subsubsection{Evaluation of the deformation}

During the whole process, we worked closely to paediatric neurosurgeons and adapted the outcomes to make the tool useful for them. The model itself provides little information a doctor can use. For that reason, we work to automatically obtain cranial measurements from the models. The measurements would give concrete information that neurosurgeons could understand and use to support the diagnosis.

\subsection{Structure of the document}

The present document is structured in nine chapters. The current chapter is an introduction that explains the problem of cranial deformation in infants. It also presents the state of the art and gives an introduction of the contents of the following chapters.

Chapter 2-7 are based on published research papers and explain the process of the development of the cranial deformation analysis tool and the present thesis. Chapters 8 and 9 are the overall discussion and conclusions sections, respectively. 
- Chapter 2: A manual photogrammetric approach for the obtainment of cranial models is presented. The methodology is based on the use of SLR cameras and it is still not applicable to the standard clinical practice. The aim of the study was to demonstrate the possibilities of photogrammetry to obtain accurate 3D models of the children's head. The obtained results were compared to the manual measurements carried out by an expert neurosurgeon. The possibility to compare the real head to ideal head's shape, given by a fitted ellipsoid is also presented.

- Chapter 3: In this chapter, the use of smartphone cameras was evaluated as an alternative to SLR high-end cameras. Smartphone cameras in slow-motion mode are useful to obtain still images of moving targets (such as the infant's heads). However, the lack of stability of the smartphone camera sensors may hamper the accuracy of the final models. Head models obtained using a smartphone were compared with models created using a SLR camera. The final models were compared by measuring distances between them. The fitted ellipsoids extracted from the models were also compared.

- Chapter 4: The study presented in this chapter has the goal of finding the best possible photogrammetric solution for the creation of the 3D models. Different parameters were evaluated, including the geometry of the images, use of targets, number of images and camera calibration methodology.

- Chapter 5: A smartphone-based, semiautomatic tool is presented. Its accuracy was evaluated by comparing the models to those acquired using CT or MRI, considered the gold standards.

- Chapter 6: An important limitation in the use of the 3D models is the registration. In this study, various methodologies were tested to assure an accurate registration of the models. Finally, the use of stickers placed by the user was the base methodology that will be used in the tool.

- Chapter 7: A fully automatic, patent-pending tool is presented. The accuracy and repeatability of the models were evaluated. Automatic linear magnitudes were computed, its accuracy and repeatability were tested.

These chapters are followed by an overall discussion and conclusions, where the whole methodology is evaluated and the future lines of development are pointed out. 


\section{2}

\section{A manual solution for the creation of head 3D models}

This chapter is based on the following paper:

Barbero-García, I., Lerma, J. L., Marqués-Mateu, Á., \& Miranda, P. (2017). LowCost Smartphone-Based Photogrammetry for the Analysis of Cranial Deformation in Infants. World Neurosurgery, 102, 545-554. https://doi.org/10.1016/j.wneu.2017.03.015

\section{$2.1 \quad$ Introduction}

In this chapter, a manual approach to extract 3D models of infants' heads is presented. The obtained models are used to carry out measurements that are later compared by manual measurements carried out by an expert neurosurgeon. The models are also fitted to an ellipsoid, representing the ideal head shape. At present, the advances in 3D techniques allow users to create accurate 3D models using non-specific technology such as consumer-grade cameras, SLR cameras, video cameras or even smartphones. This methodology can be undertaken with very low investment, depending on the camera, large/medium/small/mini frame camera, ultra-high/high speed video camera, etc. Without any doubt, image-based 3D photography is a low-cost approach compared to other highly sophisticated 3D measurement techniques such as active laser scanning and CT. In the post-processing step, 3D models allow a much more complex asymmetry evaluation than clinical examination. Three-dimensional cranial models can be fitted into geometrical models representing a so-called ideal cranial shape, such as an ellipsoid. 


\section{A MANUAL SOLUTION FOR THE CREATION OF HEAD 3D MODELS}

Using this methodology, it is possible to evaluate the deformation in terms of the wellknown Root Mean Square Error (RMSE). The RMSE has already been used by some authors to calculate the differences in shape and volume between two surfaces. These authors used RMSE to evaluate asymmetry by superposing left and right side of the head and calculating differences between them. Although the estimation of cranial volume with the assumption that it fits an ellipsoid dates back to the 1950s, as pointed out by several studies (45, 46) reported by Manjunath (47), no studies have focused in comparing the true cranial shape with an ideal mathematical model such as an ellipsoid. The aim of this study is to extend the diagnosis of cranial deformation by 3D modelling, using simple, automatic, low-cost and low-invasive techniques.

\subsection{Materials and methods}

The evaluation of the cranial deformation of patients was carried out using different methodologies. Firstly, the deformation measures were taken by a paediatric neurosurgeon during a medical consultation using a calliper. Afterwards, a 3D cranial model was created using image-based 3D modelling, namely photogrammetry, the science and technology of extracting measurements from photographs. The 3D model was analysed to measure the cranial deformation using both traditional clinical measurements (matching the measurements taken with the calliper) and automatic 3D image-based methodologies (Fig. 2.1).

\subsubsection{Planning}

Photography sessions with infants place several problems that should be solved with a suitable setup and session planning. Infants (in the range of 3-8 months) are very vivid during medical consultation and it is extremely difficult to keep them calm during the image survey. Imagery acquisition using conventional cameras usually results in badfocused images due to the movement. However, the use of anaesthesia to put the patient to sleep is a highly invasive methodology and it was avoided. Lighting conditions do need to be specifically set up for the image acquisition process, in case normal indoor illumination is not enough. On the one hand, for digital photography in the patient visit room; additional lighting in the form of halogen (or better LED) lamps was required to guarantee low ISO values on the camera systems with our digital SLR camera (Canon 


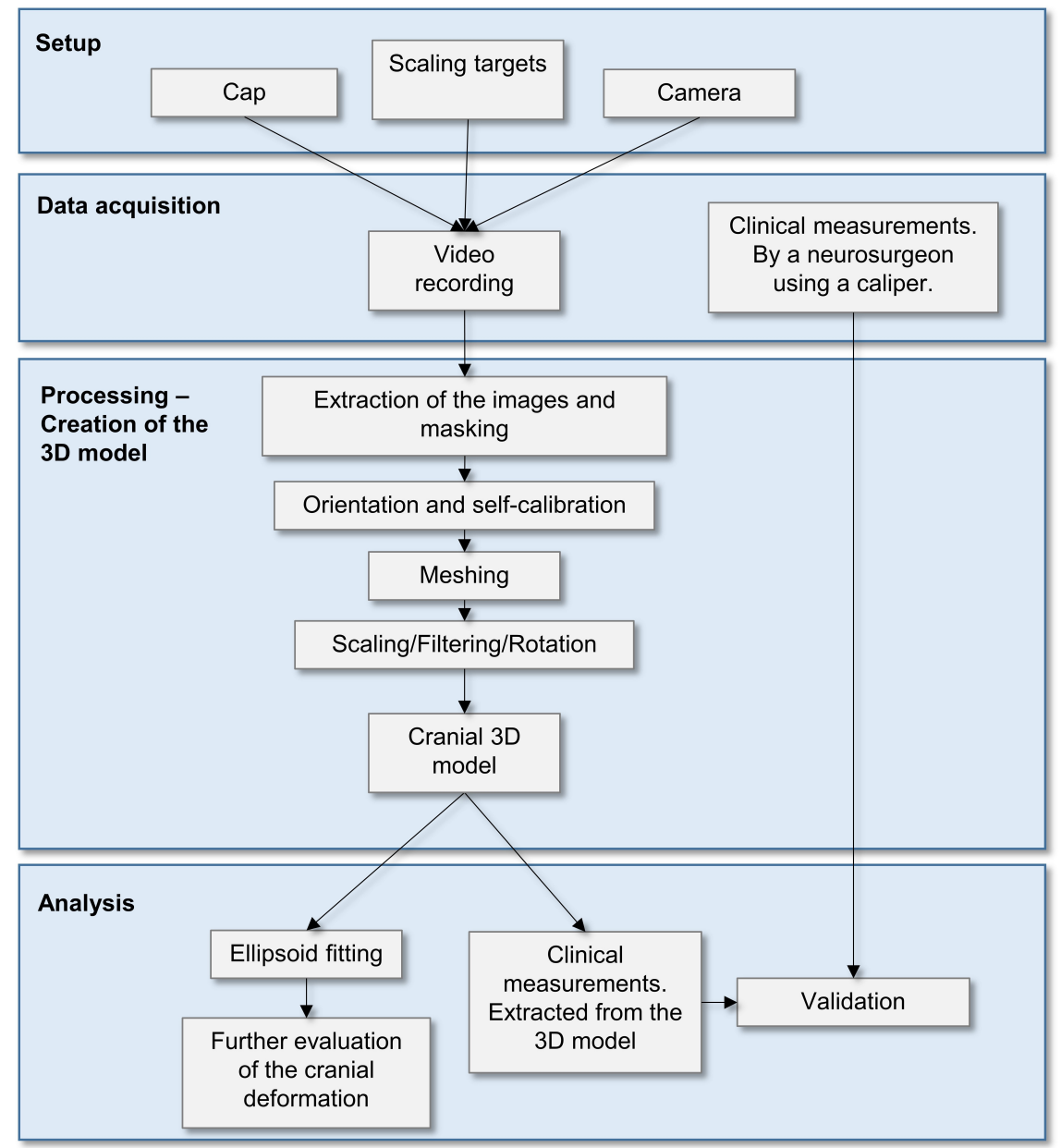

Figure 2.1: Methodology workflow 


\section{A MANUAL SOLUTION FOR THE CREATION OF HEAD 3D MODELS}

EOS 1 Ds Mark III). On the other hand, the use of videos, and specifically, slow-motion videos (smartphone Samsung S7 Edge) was found a good alternative to obtain high resolution, well-focused images even with movement. Neither special lighting lamps nor photographic devices such as tripods were required. In addition, it was found to be a quick methodology compared to taking pictures with a SLR camera. A second problem is posed by the movement, specifically, the head and the background changing position in relation to each other. This issue can be solved by using a setup of multiple synchronised cameras that take images at the same exact moment, each one from a different location around the head. This setup works well for moving targets, however, it is expensive and complex. The pre-processing step of creating digital masks can solve this problem in a simpler way making use of just one camera. A mask delimitates the area of the image that will be used for the 3D modelling and the background is, therefore, excluded. Manual masking is a time-consuming process but it can be automated to some extent. To avoid the effect of the hair on the quality of the model a cap was used. Targets were placed on the cap to improve the quality of the model and facilitate its creation with well-defined marks.

\subsubsection{Setup}

The setup was considered to be a simple and quick process. Firstly, the cap is placed (Fig. 2.2): the cap is easy to place and fits well to the cranial shape. Second, some targets are placed on the cap. These targets facilitate the creation of a 3D model and are easily identified as marks. They will also allow the scaling of the model. Different types of stickers can be used as targets as long as their size is known. For good results, targets must be evenly distributed on the cap. The illumination of the area must be acceptable. Although no special equipment is used, it is important to assure that there is enough light in the room. Prior to the video recording, the patient has to be set in place. The infant can be hold upright by an adult or be lying, provided that all the areas of the head are visible. It is also possible to interrupt the video recording and move the infant to a new position, to assure that the whole head is covered.

\subsubsection{Image acquisition}

After planning and setup, image acquisition goes on. The acquired images will be later used to obtain both accurate measurements and the 3D model. A large number 
of images were necessary to create the model without any manual intervention. The largest limitation found was taking high-quality images in movement, as the infant is rarely static. Different techniques were tested for this purpose. Standard photography provides high quality images; however, it would require a large amount of time to take all the necessary images. On the other hand, videos would not work adequately as most frames were bad-focused due to the movement of the patient. A slow-motion video taken with a smartphone was found to be the most suitable technique. Videos have been used by different authors as an input for 3D models in order to reduce the image acquisition time (48). The amount of time required was small, between 3 and 5 minutes for the preparation and video recording. The quality of the extracted images, $720 \mathrm{p}$, was proven to be sufficient for 3D modelling. Moreover, the camera worked well with moving targets and most of the frames were correctly focused. During the video session, the infant was being held by an adult; therefore, no special equipment was necessary. A special cap was used to avoid hair from affecting the model. Some measures were registered on the cap to allow the later escalation of the model. A total of 120 images were automatically extracted from the video using a fixed frequency, for instance, 1 frame out of 10 . This high number of images assured that the process would work even with some low-quality images.
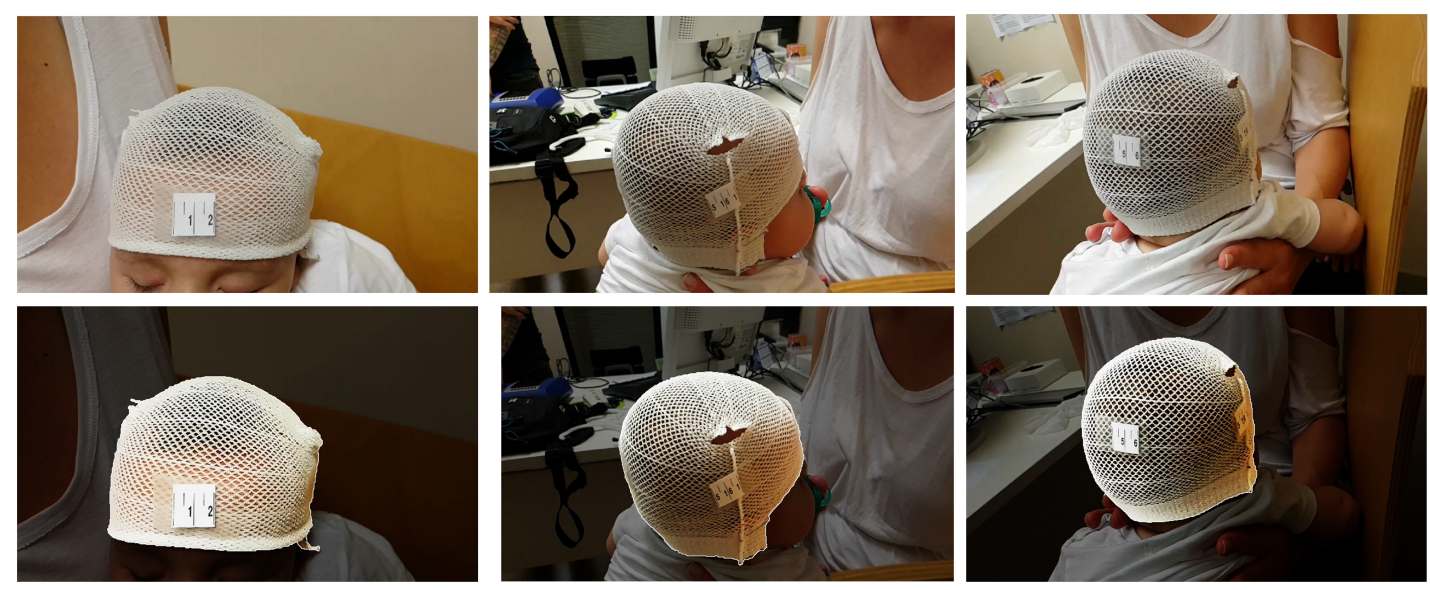

Figure 2.2: Sample of images used for 3D modelling. Without masks (top) and with masks (bottom).

In order to obtain an accurate 3D model the images must have a good geometric distribution and cover the whole head. The images were masked (Fig. 2.2), so only the 


\section{A MANUAL SOLUTION FOR THE CREATION OF HEAD 3D}

MODELS

area of interest is taken into account for processing, while the background is disregarded.

\subsubsection{Camera orientation and calibration}

In this research, the model was created using the Agisoft Photoscan software (Agisoft LLC, Russia). The first step of the process is the image orientation in 3D space, also known as alignment. In this step, the common points between images are matched, the position of the cameras is determined and the inner calibration parameters are computed. This process is highly conditioned by the texture of the object being modelled, the distribution of the marks and the geometry and quality of the images. A good geometry of the images covering the whole model is required (Fig. 2.3). Manually chosen points can be used to improve the results. However, in this case with image over-redundancy, the process was fully automatic due to the fact that masked images were used and enough image features were extracted, detected and matched among imagery. The result is a $3 \mathrm{D}$ point cloud with oriented images covering the vault of the infant (Fig. 2.3).

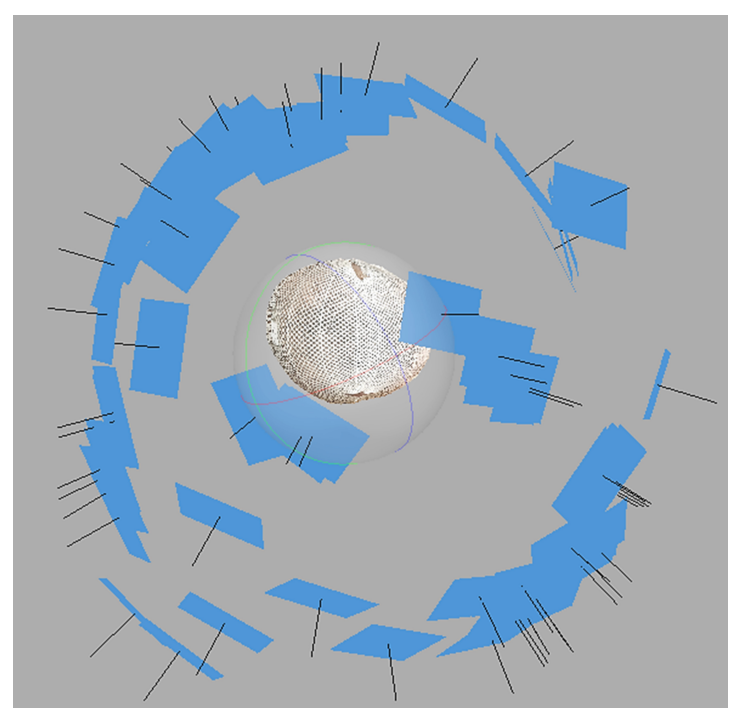

Figure 2.3: Oriented images (blue), with principal distances in 3D space (black lines) and sparse $3 \mathrm{D}$ point cloud.

Distortion affects all camera lenses and digital cameras. Especially important are radial and decentring distortion on amateur digital cameras. Fraser (49) identified up to 10 additional parameters to be considered for close-range imaging: principal 
distance (1), principal point offsets (2), coefficients of radial distortion (3), coefficients of decentring distortion (2) and in-plane camera distortions (2). The compensation of the distortion effects must be determined in order to achieve maximum accuracy and reliability in the photogrammetric results. For this particular project, the smartphone camera was self-calibrated during the project, i.e. the same images were used for the calibration as for the orientation and reconstruction following the Structure from Motion (SfM) photogrammetric pipeline. The calibration was carried out with the same software. The calibration parameters for the smartphone camera are displayed in Table 2.1. Instead of the 10 additional parameters specified by Fraser (49), 11 additional parameters were determined, increasing both the coefficients of radial and decentring distortions up to 4 . The effects of the camera calibration with all the distortions can be visualised in Figure 2.4, where a large distortion effect (up to 25 pixels) is present around the corners.

\begin{tabular}{cc}
\hline Width & 1280 \\
Height & 738 \\
PW & 0.0292834 \\
PH & 0.0292834 \\
F & 29,3963 \\
XP & -0.00026884 \\
YP & -0.00728432 \\
K1 & -0.00066592 \\
K2 & 0.394911 \\
K3 & -0.00102179 \\
K4 & $1.05584 \mathrm{E}-06$ \\
P1 & 0.000174136 \\
P2 & -0.00040031 \\
P3 & -0.00242478 \\
P4 & 0.608682 \\
\hline
\end{tabular}

Table 2.1: Calibration Parameters from PhotoScan. Values are in $\mathrm{mm}$. PW, pixel width; $\mathrm{PH}$, pixel height; F, focal; XP and YP, principal point coordinates; K1, K2, K3 and K4, radial distortion coefficients; $\mathrm{P} 1, \mathrm{P} 2, \mathrm{P} 3$ and $\mathrm{P} 4$, tangential distortion coefficients.

It is worth noticing that if more than one camera is used for the data acquisition, 


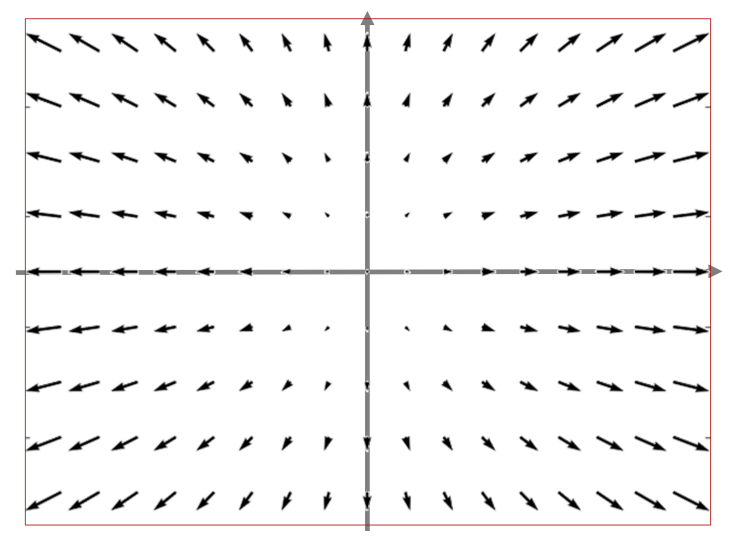

Figure 2.4: Camera distortion grid

different calibration parameters will be achieved and applied to correct the image distortions. Furthermore, if the settings of the camera change, such as the zoom, the camera must be recalibrated because the distortion parameters change.

\subsubsection{Creation of the 3D model}

Once the images are correctly oriented in 3D (Fig. 2.3) and calibrated, a sparse point cloud is derived (Fig. 2.5 ). After image matching, a dense point cloud can be achieved (Fig. 2.5p). For this particular project with 120 images, the point cloud had approximately $60.000 \mathrm{XYZ}$ coordinates. The dense point cloud was finally used to create an uncleaned 3D mesh (Fig. 2.5F); the mesh is formed by the vertexes and faces of the model, the faces were obtained by triangulation of the dense point cloud. This latter mesh was scaled, smoothed and rotated (Fig. 2.5d). The scaling process was carried out using reference marks placed on the cap, the marks were distributed around the whole cap to minimize the errors. Three distances were measured for the model and the obtained accuracy was better than $1 \mathrm{~mm}$. The model was smoothed to remove small imperfections, the cap texture and other details that do not provide any useful information to the model.

The last step is texturing this mesh for visualisation purposes (Fig. 2.6). The images need to be correctly registered. For each polygon of the mesh, the texture is mapped from the best-quality image visible from it. The images are normalised to avoid changes in illumination. 

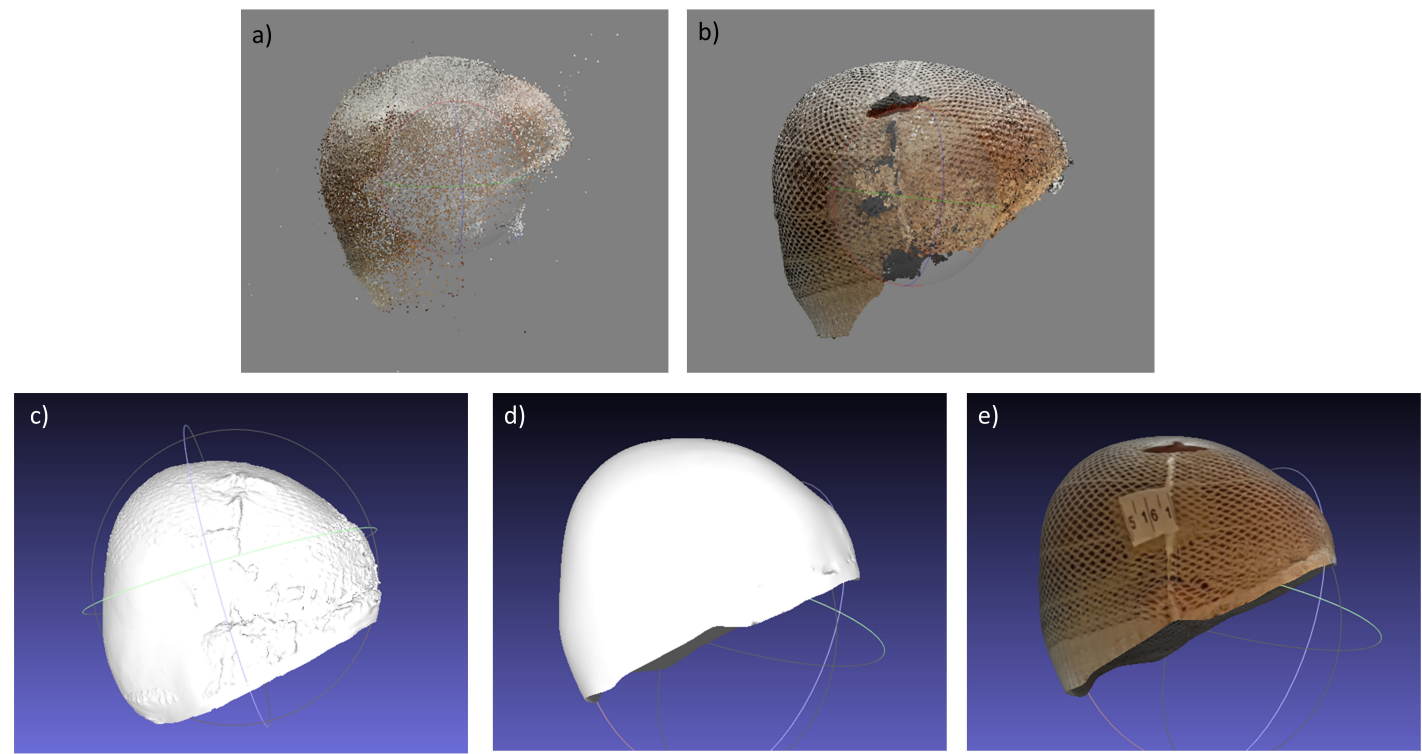

Figure 2.5: Model creation steps : a) Tie points. b) Dense point cloud. c) Mesh. d) Mesh after smoothing.
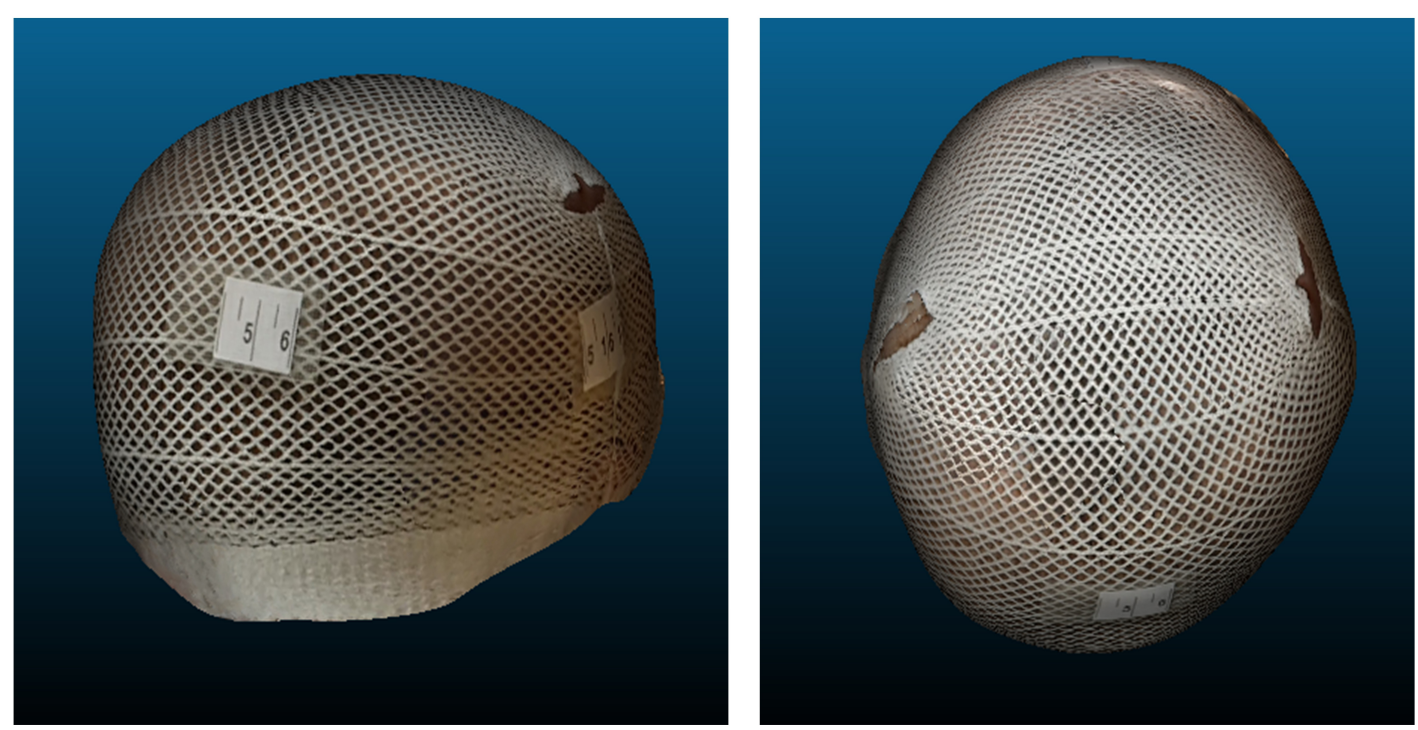

Figure 2.6: Textured mesh. 


\subsubsection{Extraction of clinical measurements}

Clinical measurements (Fig. 2.7) were obtained as part of the routine assessment of the patients, during the same session as the video recording. These measurements are used to calculate different indexes that are used as a measure of deformation. In this case, the measurements extracted during the medical consultation were compared with those extracted from the 3D model in order to evaluate the methodology.

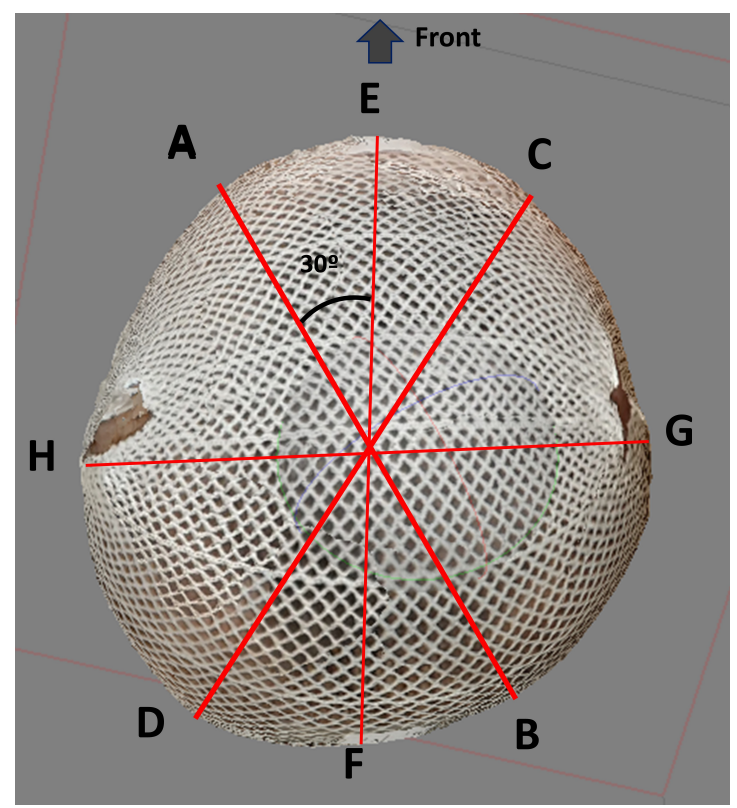

Figure 2.7: Diagram of reference for clinical measurements.

\subsubsection{Ellipsoid fitting}

The use of 3D data models enables new possibilities, such as the comparison between the model and other standard surfaces. The ellipsoid has been identified as the perfect head shape, as pointed out for the estimation of cranial volume by several experts (47). However, we are not interested herein in determining volumes, but ideal geometric cranial shapes. Following this way, it is possible to accurately compute and characterize the deformations as differences between the deformed cranial head and the ideal fitted ellipsoid that will be used as a supporting surface. The image-based 3D textured model was rotated according to the EF axis (Fig. 2.7). An ellipsoid was created to fit the points in the $3 \mathrm{D}$ model. The mathematical model to fit the ellipsoid can be found 
in Bektas (50). Instead of the geometric distance, a more robust one, the orthogonal distance from each point to the ellipsoid, was calculated as pointed out by Bektas (51). The results are shown as hypsometric colours in the 3D model (Fig. 2.9).

\subsection{Results}

Firstly, we conducted this systematised image acquisition for a patient with positional plagiocephaly: a six-month-old child who showed a neat occipital flattening despite previous rehabilitation treatment. The two sets of clinical measures taken with the calliper and using the 3D model were compared 2.1. Important differences can be found between the two methodologies. The differences in the perimeter measures are small (2 $\mathrm{mm}$ ); however, the distances of the diagonals show higher differences (up to $19 \mathrm{~mm}$ ). The large differences among measurements are thought to be due to wrongly chosen cranial marks. As there is no possible way to infer the position of the bones on the $3 \mathrm{D}$ model it is difficult to choose the correct points for measurement. Small differences in the position of these points will cause large differences in the measurement of the distances.

\begin{tabular}{ccc}
\hline & Caliper & 3D Model \\
\hline Perimeter & 450 & 452 \\
AB & 130 & 147 \\
CD & 145 & 152 \\
EF & - & 155 \\
HG & - & 131 \\
Arc CD & 240 & 222 \\
Arc AB & 250 & 231 \\
\hline
\end{tabular}

Table 2.2: Clinical Measures Taken with Calipers and 3D Models. Values in mm.

The distances between the cranial 3D model and the fitted ellipsoid were also calculated, the mean distance is close to zero, the highest difference is $5.8 \mathrm{~mm}$ (Table 2.3 .

In order to analyse the cause of the encountered differences, four more patients were evaluated. For some patients some of the cranial marks were placed on the patient's cap to assure that the correct points were measured (Fig. 2.8). The results showed great 


\begin{tabular}{ccc} 
& Distance to the Ellipsoid & Global Asymmetry \\
\hline Mean & -0.2 & 2.4 \\
Max & 4.6 & 9.2 \\
Min & -5.8 & -1.5 \\
SD & 2.3 & 2.2
\end{tabular}

Table 2.3: Distances between cranial model and the ellipsoid compared to global asymmetry.

differences among measurements with well-defined points and measurements where the points were not perfectly located. Measurements with well-defined marks include EF distance for all the patients and HG distance for two of them. For these measurements differences are between 1-3 mm, being the distances slightly larger for the 3D model (Table 2.4).

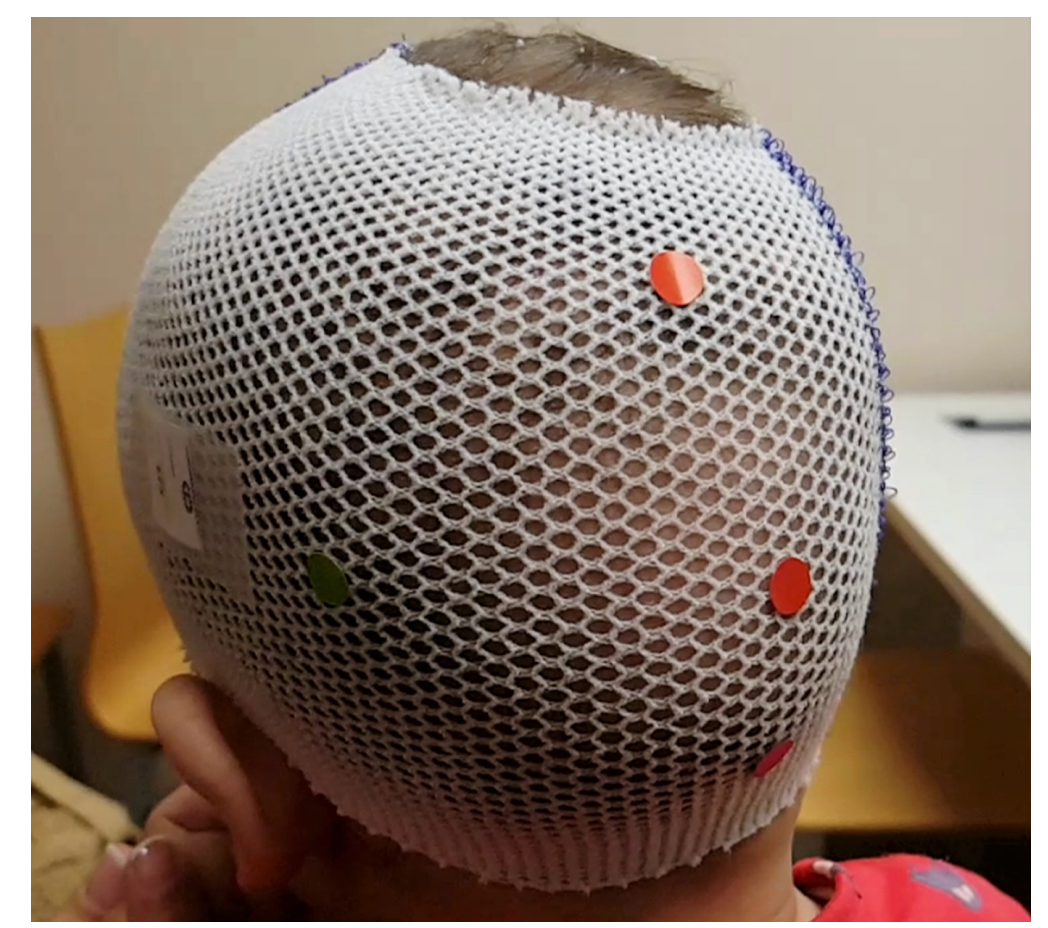

Figure 2.8: Marks placed on the patient cap

For the rest of the measurements (without well-defined marks) differences are much higher. It was also noted a high variability when these measurements were repeated several times for the same model. 


\subsection{Discussion}

\begin{tabular}{ccccccccc} 
& \multicolumn{2}{c}{ Patient 2 } & \multicolumn{2}{c}{ Patient 3 } & \multicolumn{2}{c}{ Patient 4 } & \multicolumn{2}{c}{ Patient 5 } \\
\hline & Caliper & 3D Model & Caliper & 3D Model & Caliper & 3D Model & Caliper & 3D Model \\
\hline Perimeter & - & - & - & - & 465 & 495 & 430 & 441 \\
AB & - & - & - & - & 135 & 138 & 145 & 149 \\
CD & - & - & - & - & 154 & 159 & 144 & 145 \\
EF & 155 & $156^{*}$ & 145 & $148^{*}$ & 154 & $156^{*}$ & 150 & $151^{*}$ \\
HG & 115 & $118^{*}$ & 120 & $122^{*}$ & 130 & 141 & 115 & 122
\end{tabular}

Table 2.4: Clinical measurements for patients two to five. ${ }^{*}$ Measures with points marked on the cap.

\subsubsection{Visualization}

The 3D visualisation of the cranial deformations provides a useful tool for the better understanding of the numerical results. The deformations can be visualised as the hypsometric colours on the model, given by the distance to the ideal ellipsoid (Fig. 2.9). The maximum deformations are also marked on the model. For this research, visualisation was carried out using the open-source software CloudCompare 2.7.0. However, the whole process was also automated in-house using Python scripts. The resulting model is exported to Wavefront OBJ format, allowing its visualisation in a wide range of software packages.

\subsection{Discussion}

The differential diagnosis of cranial deformities is one of the most common problems attended by paediatric neurosurgeons in infants. While craniosynostosis is considered a serious disease requiring surgery in most cases, positional plagiocephaly and brachycephaly are perceived as minor deformations and may be frequently overlooked and given little attention. The large number of benign cases, however, provides us the chance to refine the methodology of our assessment. Ideally, we should be able to obtain real and interactive 3D models in a simple and easy way, using low-cost devices, and in short periods of time. Instead of 3D photography, different methodologies can also be applied to create a 3D model, such as the use of triangulation-based 3D scanners (specially developed for this purpose or created for a more general use) or CT scanners. However, CT scanners can involve risks for the newborns health, as 


\section{A MANUAL SOLUTION FOR THE CREATION OF HEAD 3D}

MODELS
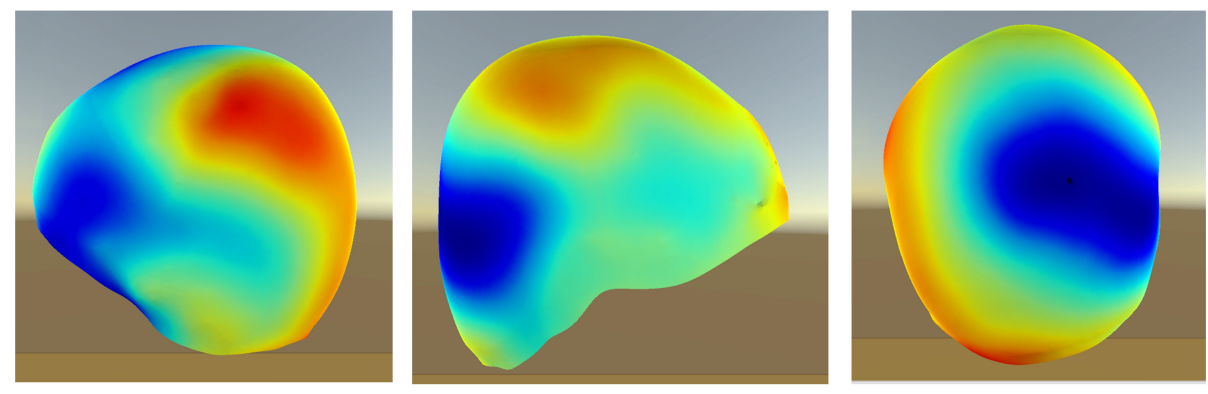

\section{Legend}
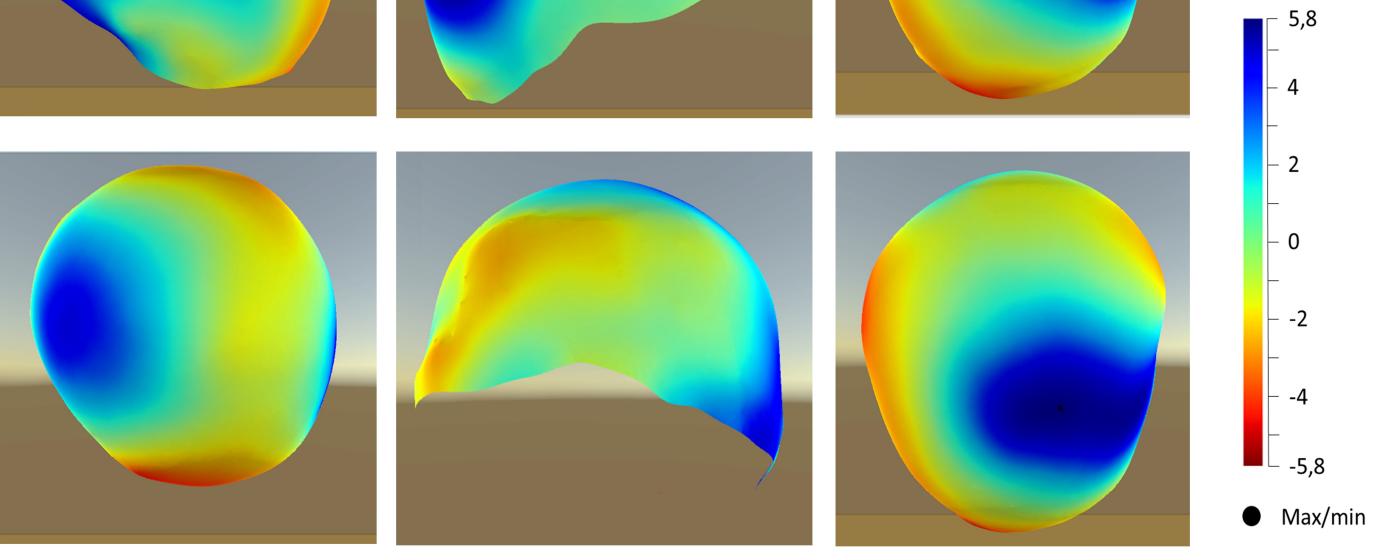

Figure 2.9: Ellipsoid fitting differences (mm): a) Left view, b) Right view, c) Posterior view, d) Superior view, e) Frontal view, f) Anterior-superior view.

they increase the risk of tumours (52). From our experience, common low-cost handheld self-positioning 3D scanners did not fit for this purpose due to the quick head movements of the infants, failing the creation of the 3D model. In addition, the 3D models did not achieve the required accuracy; specialised structure-light 3D scanners involve high costs and were discarded for the experimentation. Photogrammetry, in the form of slow motion image-based 3D solution, was found to be the most suitable technique for model creation, as it is low-cost and low-invasive. Analysing the results, the possibilities of any accurate 3D model are huge compared to traditional methods. The traditional methodology using callipers usually includes between 5 and 7 direct measurements. In contrast, the 3D methodology includes between 30.000 and 40.000 points, from which a 3D mesh is achieved. The deformation can be measured considering the resulting mesh. Compared to the CT scanner, the advantages include not only avoiding radiation but also obtaining similar results for the external part, at a smaller cost. For the first patient, important differences are encountered between the clinical measurements obtained with the calliper and the same measures extracted from the 3D model. Only the perimeter obtained using both methodologies is very similar, with 
a difference of only $2 \mathrm{~mm}$ (Table 2.2). Nevertheless, for the rest of the patients $(4$ out of 5), results show small measurement differences as far as well-defined marks are set by the paediatric neurosurgeon before data acquisition (check Fig. 2.8). This fact about marks placement before photogrammetric data acquisition is extremely relevant. Distances measured (EF \& HG, Table 2.4) on the 3D model are slightly larger (1-3 $\mathrm{mm}$, mean $2 \mathrm{~mm}+/-0.9 \mathrm{~mm}$ ) than those measured directly on the patient. The over estimation in the 3D model is in accordance to other authors $(52,53)$ and can be due to either the pressure applied while using callipers or the use of skullcaps (53). For measurements without well-defined marks, important differences among measurements are encountered. The difficulty to correctly identify cranial marks can be considered both a limitation of the modelling solution and a requirement to obtain reliable measurements, as the possibility to manually carry out distance measurements is unlimited within the 3D model. Thus, it can be stated that these marks must be placed on the head prior to video recording. Alternatively, new software development that mathematically defines key points $(\mathrm{A} . \mathrm{H})$ is foreseen as a good empirical solution to determine maximum and minimum cranial distance measurements, in a way to avoid marking. The ellipsoid fitting approach offers advantages compared to other 3D measurements, such as the global asymmetry, which compares the head shape with non-mathematical surfaces. Instead, the ellipsoid is a mathematical surface and consequently, orthogonal distances to the ellipsoid can be calculated. Orthogonal distances are more robust than geometric distances, therefore, the ellipsoid fitting approach is recommended in order to achieve reliable and accurate results. The ellipsoid fitting approach presented in this article was constrained. It means that one of the semiaxis directions matched the EF axis of the head (Fig. 2.7). This was found necessary as the cranial asymmetry computation can result in a wrongly rotated ellipsoid. With an incorrectly oriented ellipsoid, the calculated deformations would be smaller, but unreal. Therefore, the ideal head shape is an ellipsoid with one of its semiaxis matching the ideal head symmetry plane.

\subsection{Conclusion}

Photogrammetry and 3D modelling are highly useful tools for the measurement of cranial deformation. It has been proved that it is possible to create an easy, low-cost, low-invasive and accurate methodology to measure plagiocephaly, brachycephaly and 


\section{A MANUAL SOLUTION FOR THE CREATION OF HEAD 3D}

MODELS

craniosynostosis with 3D photogrammetric solutions. Smartphones as a working device (far beyond 2D imagery) allow doctors to undertake rigorous 3D measurements as far as marks are available on-site, independently of the medical centre, hospital or clinic. The acquisition of such objective data is essential for establishing correct diagnosis and monitoring outcomes in deformational plagiocephaly and craniofacial pathology. A new technique has been pointed out to determine cranial deformation based on ellipsoid fitting, particularly, the measurement of orthogonal distances from the real head surface to an ideal ellipsoid. The main areas for future research and development are related to the automation of some parts of the processing, such as masking or scaling, and the integration of the whole process in a single software package. Following this way, a low-cost photogrammetric analysis toolbox could be easily available for the assessment of our patients in both hospitals and medical clinics, improving our diagnostic accuracy and facilitating an objective follow-up. A validation of the methodology presented herein will be carried out in the near future to compare 3D models by means of photogrammetry and CT. 


\section{3}

\section{An smartphone-based application}

This chapter is based on the following paper:

Lerma, J. L., Barbero-García, I., Marqués-Mateu, Á., \& Miranda, P. (2018). Smartphonebased video for 3D modelling: Application to infant's cranial deformation analysis. Measurement, 116, 299-306. https://doi.org/10.1016/j.measurement.2017.11.019

\subsection{Introduction}

As stated in Chapter 1, photogrammetry and 3D modelling are non-invasive tools that provide more detailed data than calliper measurements. However, the cost of the photogrammetric processes depends highly on the setup. One of the main challenges for the measurement of cranial deformation in infants is the movement of the patients. Infants, between 2 and 8 months old are, in most cases, in constant movement during the consultation, and below three months, they can hardly hold their heads. Therefore, special care must be taken to assure that the images are well-focused. The use of SLR professional cameras, although possible in some cases (i.e. the infant is extremely calmed), is usually challenging, it requires time and additional lighting sources. On the other hand, the use of a single portable triangulation 3D scanner, such as Sense 3D (3D Systems, U.S.), is even more limited by the infant's movement and does not work with this type of real-life settings. To deal with the problem of the movement, some photogrammetric techniques include complex setups of several professional cameras or a combination of cameras and $3 \mathrm{D}$ scanners. For these solutions, costly equipment is required. The use of smartphone cameras as a tool to create high-accuracy $3 \mathrm{D}$ models 


\section{AN SMARTPHONE-BASED APPLICATION}

is becoming more common as the capabilities of the smartphones increase and they are equipped with higher quality cameras (54, 55). Smartphone-based photogrammetry is starting to be used in different medical studies, most of them focused on the creation and measurement of the medical prosthesis (38, 56). However, in these studies, the target is static and photographs are used instead of video sequences. The use of videogrammetry, an imaging technique that extracts 3D measures from video, is also becoming more common as it allows the acquisition of a large number of well-focused images in short periods of time, even with moving targets (57). Videogrammetry is usually understood as the science to extract 3D coordinates as a function of time, allowing the obtainment of multitemporal data of dynamic objects (58, 59). In this particular study, there is no intention to obtain dynamic information but well-focused images of a non-changing moving target. Videogrammetry reduces the time required for image acquisition and the high number of frames allows the removal of badly focused images (48). Smartphone-based slow-motion videos are an especially interesting methodology which allow us to obtain focused images of moving targets easily. The aim of this chapter is to compare the results obtained using photographs taken with a digital SLR camera and those obtained using a slow-motion video recorded with a smartphone by means of an auxiliary ellipsoid shape fitting the head. The use of a Sense 3D scanner was also part of the initial experiment. However, it was not possible to obtain a model of the infant using this device and therefore, the results achieved with the triangulation laser scanner are not reported here. Nevertheless, the experiments undertaken will indicate whether slow-motion videos taken with a smartphone can provide a similar accuracy than SLR cameras for the measurement of cranial deformation in infants or not.

\subsection{Methodology}

Two 3D models of the head were created for the same patient using two imaging sensors: an Advanced Photo System type-C (APS-C) frame SLR camera and a high-end smartphone in slow-motion video mode; a set of frames was later extracted from the video. Parallel processes were carried out to create both models. The images were masked and orientation and self-calibration processes were carried out. Once each mesh was obtained, it was cleaned and smoothed. Lastly, the fitted ellipsoid was calculated for 
each mesh using least squares adjustment and the ellipsoids and meshes were compared to evaluate their differences (Fig. 3.1). The acquisition of well-focused images to create the 3D model using the SLR camera was possible due to a situation more favourable than usual (i.e. the infant was especially calmed). Nevertheless, image acquisition lasted longer than the video recording.

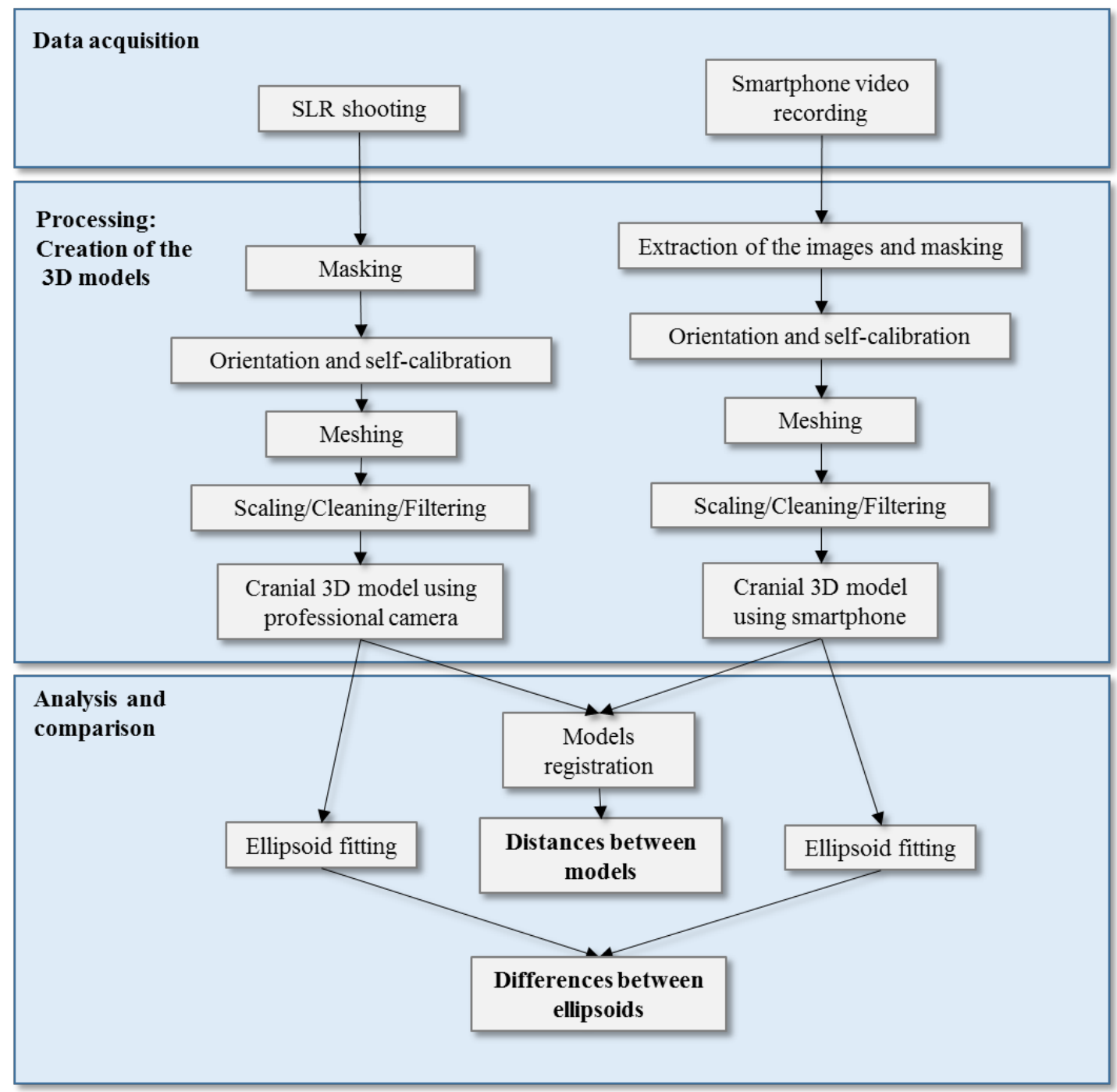

Figure 3.1: Flow diagram. 


\section{AN SMARTPHONE-BASED APPLICATION}

\subsubsection{Setup}

The image acquisition was carried out during a standard medical consultation, which involves several limitations. The time slot is very limited as the doctor has to move to the next patient within minutes. The infant (normally aged between 2 and 8 months old) is usually awake and moves quickly during the process and, in many cases, he gets nervous when he is set on an ideal position to take the pictures. In addition, patient's parents are usually present and a certain degree of nervousness is to be expected. In this situation, it is vital to be quick, straightforward and interfere the minimum with the consultation. The setup for the data acquisition was therefore kept as simple as possible. This assures that the cost related to imaging equipment is expected to be low and the processes can be carried out regardless of the consultation place. The image shooting and the video recording were carried out during the same consultation. The infant was held by an adult, therefore no special equipment was required. A cap was placed on the children's head to avoid hair from affecting the quality of the model. Four pieces of measuring tape, of approximately two centimetres were placed on the cap. These marks were included in the model to allow scaling the model and to facilitate the image registration with easily identifiable features. In order to simplify the process no special light conditions were required, the usual illumination of the consultation room was enough.

\subsubsection{Data acquisition}

Two imaging devices were used for data acquisition (Fig. 3.2). First, a digital SLR camera Canon EOS 1100D was used at a maximum resolution of 4272 x 2848 pixels with a Canon lens EF-S 18-55 mm. A principal distance of $35 \mathrm{~mm}$ was selected for taking the pictures at ISO 3200.No tripod or special illumination was used. Second, a high-end smartphone Samsung Galaxy S7 Edge (Fig. 3.2p) in slow-motion video was used at a resolution of $1280 \times 738$ pixels (Table 3.1. .

0.8

It has been reported that good-quality 3D models can be obtained using approximately 10-20 images for outer dome surfaces (geometry close to the infant's head) using multi-convergent images (60). In this study, a higher number of images was acquired to assure that the final model can be created. A high number of images was found to be 


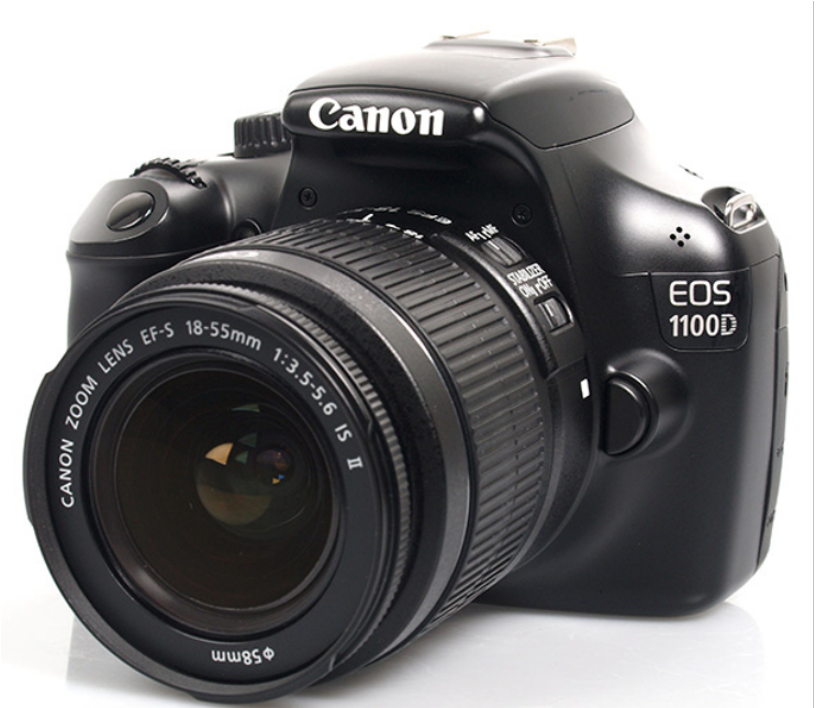

(a)

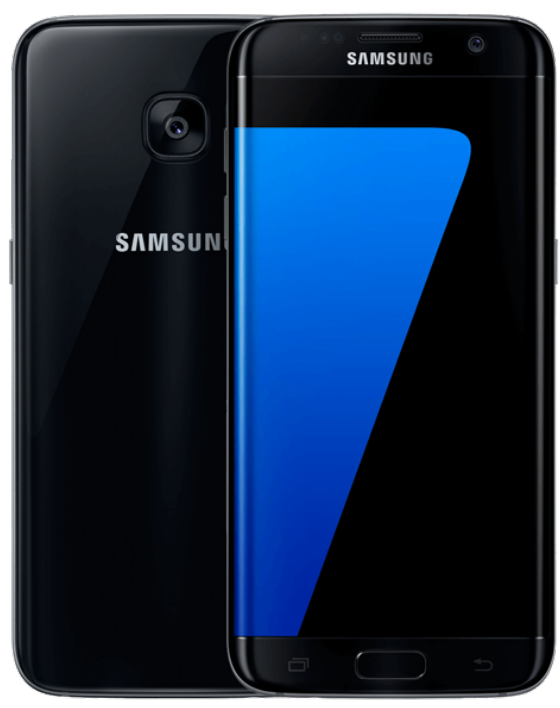

(b)

Figure 3.2: Cameras used during the image acquisition : (a) Canon EOS 1100D, and (b) Samsung Galaxy S7 Edge.

\begin{tabular}{|c|c|c|c|c|}
\hline Camera & $\begin{array}{l}\text { Focal length } \\
\qquad(\mathrm{mm})\end{array}$ & $\begin{array}{l}\text { Effective format } \\
\quad \text { size }(\mathrm{mm})\end{array}$ & $\begin{array}{l}\text { Pixel count } \\
\quad \text { (pixels) }\end{array}$ & $\begin{array}{l}\text { Pixel pitch } \\
\quad(\mathrm{mm})\end{array}$ \\
\hline Canon & 34.35 & $22.17 \times 14.78$ & $4272 \times 2848$ & 0.00519 \\
\hline \multicolumn{5}{|l|}{ EOS 1100D } \\
\hline Samsung & 4.2 & $5.64 \times 4.23$ & Video: $1280 \times 738$ & 0.0014 \\
\hline Galaxy S7 & & & Still: $4032 \times 3024$ & \\
\hline
\end{tabular}

Table 3.1: Sensor specifications.

necessary as the geometry of the network is not ideal for calibration (i.e. the infant's head occupies only the central part of the image). Another unexpected issue was that many of the images were blurred due to the quick infant's head movement. Figure 3.3 displays a partial view of the two data sets.

\subsubsection{Processing}

The two data sets were processed independently using Photoscan Professional version 1.2.6 (Agisoft LLC, Russia). The first dataset was composed by the images taken with the SLR camera once the blurry images were discarded. A large number of images were 


\section{AN SMARTPHONE-BASED APPLICATION}
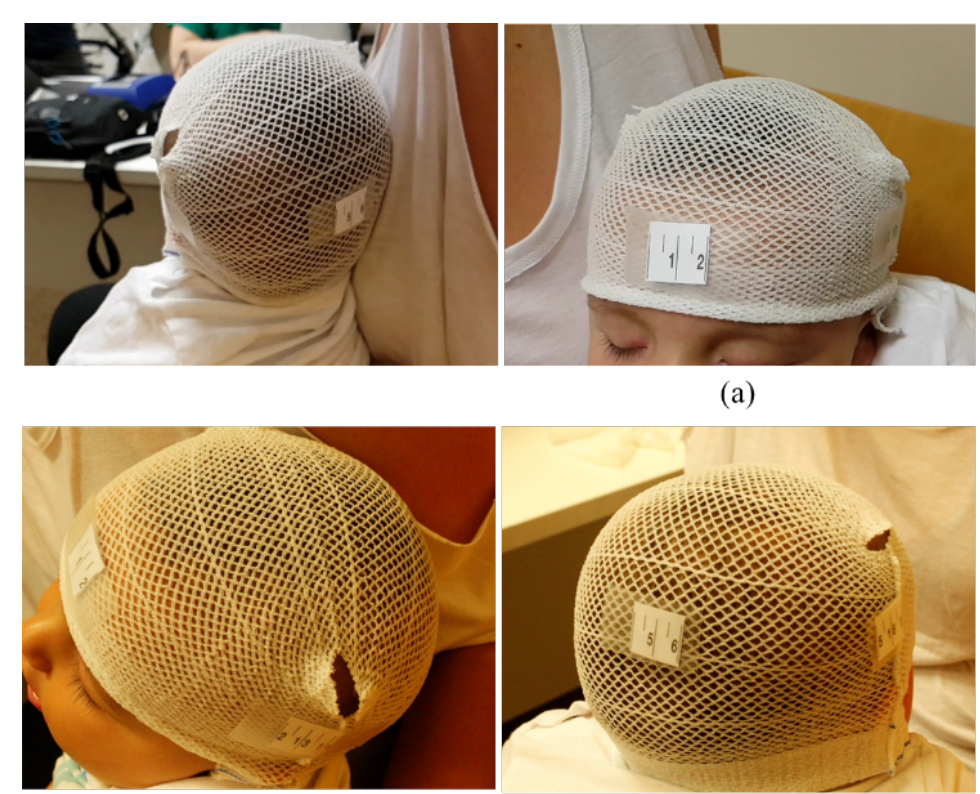

(b)

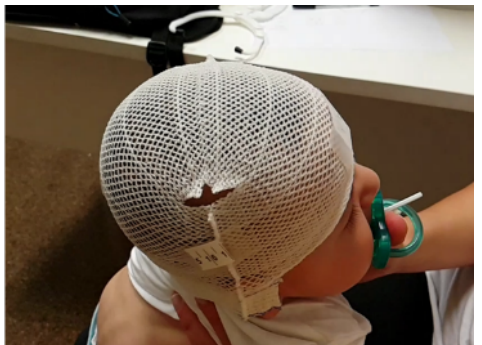

(a)

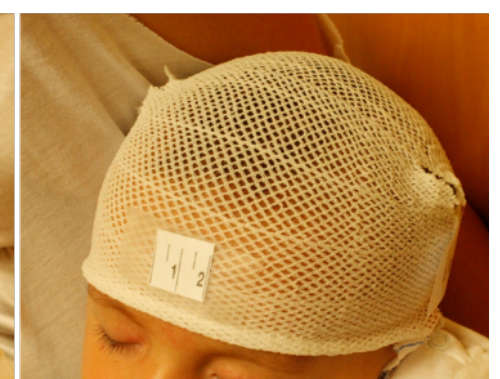

Figure 3.3: Sample of images used for the processing and 3D modelling : (a) extracted from the slow-motion video; (b) taken with SLR camera.

obtained as the elimination rate was expected to be high due to the head movement. Finally, 24 images out of 53 were used. The second set was composed of the images extracted from the video. The extraction of the most useful frames from the video is one of the main challenges in videogrammetry. The usage of the totality of the images is unnecessary and would be unmanageable in terms of computational cost. The selected images must be well-focused and present a good geometry around the object. In this case, a set of images were extracted automatically using a fixed frequency (e.g. one frame out of ten). Later, the blurred images were automatically removed using an automated procedure. The individual image quality was estimated and the bad-quality images were determined using a threshold. Finally, the geometry of the network was reviewed to manually remove redundant images. In the end, a total of 63 frames were used for the 3D modelling from the second set. The quantity of frames is almost three times larger than the number of images used for the SLR camera model. A larger number of images is necessary a priori to compensate both the lack of resolution and the geometric accuracy of the camera. The two sets of images followed the same steps to create the 3D models (Processing part, Fig. 3.1). Firstly, the images were 
masked to delimit the part of the images that were to be used for modelling. This process is necessary as the infant is moving in relation to the background. For that reason, it would not be possible to obtain an acceptable model without excluding this background through the image masks. The masking process was carried out manually, although the automation of the process must be approached in the future. The second step was the image orientation (also known as alignment), which is the process used to determine the positions and rotations for each image in the object space. During this step, the cameras were also calibrated (see below the Results Section). A relative ground reference system (GRS) for the models was set by assigning coordinates to three points of known distances. This process was carried out automatically using Agisoft PhotoScan. Later, the point cloud was densified and the mesh created. The meshes needed to be cleaned and smoothed in order to remove the texture of the cap and possible artefacts. Lastly, the final models were scaled using the markers placed on the cap and the texture was draped on the 3D models (Fig. 3.4)

\subsubsection{Self-calibration}

No independent calibration of the cameras was carried out as it would make the setup more complex and would greatly increase the time required by the doctor to collect the necessary data. Instead, a self-calibration process was undertaken with ten additional parameters. The self-calibration accuracy is expected to be limited by the nature of the setup. In most images, the object covers only a small area in the centre of the image, while the rest of the image must be masked. The inner instability of the smartphone camera is also expected to affect the calibration. Ellipsoid fitting The similarity in shape and volume between the human skull and the ellipsoid has been pointed out in previous studies (46). According to this study, the cranial deformation can be measured in terms of distances from the real head surface to an ellipsoid representing the ideal head surface (61). The ideal head shape will be different for each patient and, therefore, the ellipsoid must be calculated for each one. The ideal ellipsoid is the one which minimises the distances to the real head while meeting some constraints to assure that the head is normal, i.e. anthropometric cranial indexes considered normal according to medical literature (62). The ideal ellipsoid was obtained by least-square fitting using the mathematical model presented in Bektas (50). The obtained parameters are the semiaxes of the fitted ellipsoid. The accuracy of the solution can be evaluated using the 


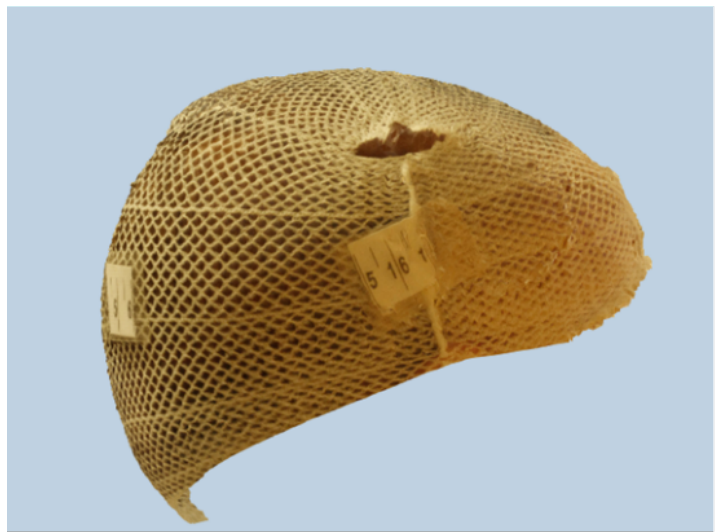

(a)

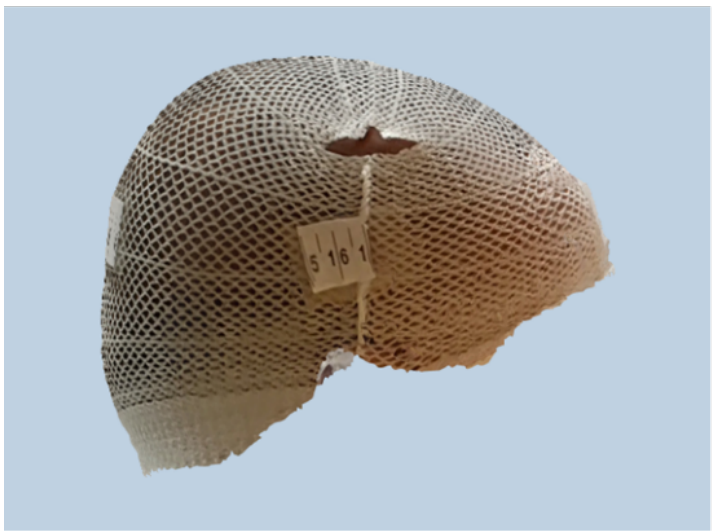

(c)

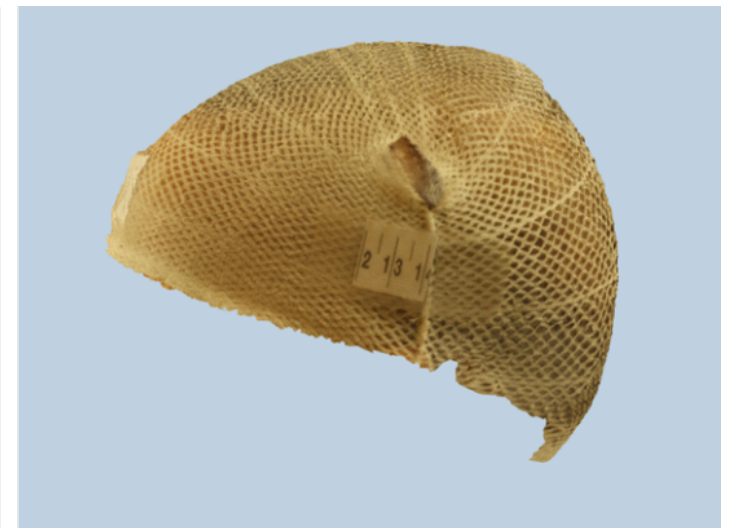

(b)

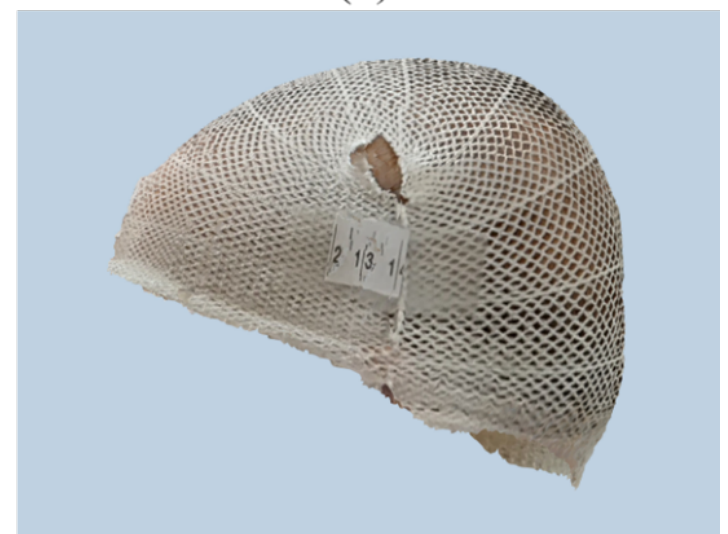

(d)

Figure 3.4: 3D models created using both cameras: (a) right and (b) left views, Canon EOS 1100D ; (c) right and (d) left views, smartphone video. 
semiaxes standard deviation (Table 3.4). Approximately 30000 points from each model were used for the fitting process. Comparison The estimated accuracy was calculated for both cameras (Table 3.2 using the formula presented in (63)

$$
\sigma=\frac{q}{\sqrt{k}} S \sigma_{i m g}
$$

The theoretical precision $\sigma$ is affected by the image co-ordinate standard error $\sigma$ $\mathrm{img}$, the image scale $\mathrm{S}$ and the number of images $\kappa$. The factor $\sigma$ img is obtained as the maximum reprojection error provided by Agisoft PhotoScan.

The design factor $q$ is given by the strength of the camera network. For acceptable convergent networks, it is expected to take values from 0.4 to 0.8 . Values of 0.6 and 0.7 are considered suitable for design purposes in these cases (64). A value of 0.6 has been assigned for both models as the network geometry is convergent. Since the goal of the study is the comparison of cameras and not the retrieval of absolute accuracy values, the value of this parameter will not affect the results, as the network geometry is the same for both cameras. Once both models were created they were compared to evaluate their differences. At this point, the models were roughly registered, however, small differences could be expected as only 3 points were used to set the relative GRS. Then, the registration was improved after identifying homologous points between the models in CloudCompare 2.7.0. working environment (GPL software). Afterwards, the Iterative Closest Point algorithm was applied to achieve the best possible accuracy in the registration. After that, the same software was used to compute the Euclidean distances between both models (i.e. the model created using the smartphone and the model created using the SLR camera) (Fig. 3.6). Following this way, the differences between models can be evaluated for each point. The evaluation of these differences will allow us to state whether the models are comparable or not. The differences between the fitted ellipsoids were compared as differences in their three semiaxes.

\subsection{Results}

Table 3.2 shows the calibration parameters and their standard deviations for both cameras. The significance of the additional parameters was evaluated using the Student's t-test $(60,65)$. The standard deviations achieved are extremely high for the smartphone camera. In fact, Student's t-test confirms that only the principal distance parameter 


\section{AN SMARTPHONE-BASED APPLICATION}

(f) is reliable. High standard deviations discredit the mean parameters during the selfcalibration, and thereby they should not be taken into account. For the SLR camera, standard deviations were considerably smaller. The radial correction parameter k1 presented a low significance value and therefore it will not be taken into account.

\begin{tabular}{ccccccc} 
& \multicolumn{3}{c}{ Samsung Galaxy S7 } & \multicolumn{3}{c}{ Canon EOS 1100D } \\
\hline & Mean & Std & t-Student & Mean & Std & t-Student \\
\hline f & 29.08 & 2.41 & $100 \%$ & 37.43 & 0.22 & $100 \%$ \\
x0 & 1.23 & 0.87 & $78.2 \%$ & 1.29 & 0.03 & $100 \%$ \\
y0 & 0.70 & 2.17 & $23.8 \%$ & -0.16 & 0.02 & $100 \%$ \\
k1 & $-4.2 \cdot 10-04$ & $8.1 \cdot 10-04$ & $36.8 \%$ & $-2.9 \cdot 10-05$ & $2.2 \cdot 10-05$ & $74.7 \%$ \\
k2 & $1.4 \cdot 10-06$ & $4.9 \cdot 10-06$ & $20.9 \%$ & $-2.9 \cdot 10-06$ & $1.2 \cdot 10-07$ & $100 \%$ \\
k3 & $-1.7 \cdot 10-09$ & $7.5 \cdot 10-09$ & $16.6 \%$ & $3.0 \cdot 10-09$ & $1.7 \cdot 10-10$ & $100 \%$ \\
p1 & $-4.2 \cdot 10-04$ & $8.5 \cdot 10-04$ & $34.9 \%$ & $2.6 \cdot 10-04$ & $3.5 \cdot 10-06$ & $100 \%$ \\
p2 & $-7.0 \cdot 10-04$ & $9.5 \cdot 10-04$ & $50.2 \%$ & $1.9 \cdot 10-04$ & $6.2 \cdot 10-06$ & $100 \%$ \\
b1 & 0.04 & $9.4 \cdot 10-03$ & $98.9 \%$ & $-2.8 \cdot 10-03$ & $5.3 \cdot 10-05$ & $100 \%$ \\
b2 & $9.1 \cdot 10-03$ & $8.5 \cdot 10-03$ & $46.2 \%$ & $-4.9 \cdot 10-03$ & $3.1 \cdot 10-04$ & $100 \%$
\end{tabular}

Table 3.2: Additional calibration parameters for the two cameras (f, $\mathrm{x} 0$, y0 in $\mathrm{mm}$ ), standard deviations and significance percentage according to Student's t-test.

The great differences in the image deformations can be graphically observed in Figure 3.5. As may be expected, both radial and tangential distortions are much higher for the smartphone camera. In spite of these values, if the corrections are correctly calculated and applied, the final geometric camera calibration model will cope with the deformation and the observations will be free of systematic errors thanks to the interior orientation parameters.

The theoretical precision and the relative accuracy, together with the parameters that affect them, can be checked in Table 3.3. The standard deviation of the image measurements $\sigma_{i m g}$ is slightly better for the SLR camera. Nevertheless, the final estimated precision is slightly better for the smartphone camera. This difference is caused mainly by the larger quantity of images obtained and used with the smartphone. The differences in precision can be considered not relevant.

Figure 3.6 shows several views and a histogram with the Euclidean distances between the two 3D models. These distances are represented as hypsometric colours on 


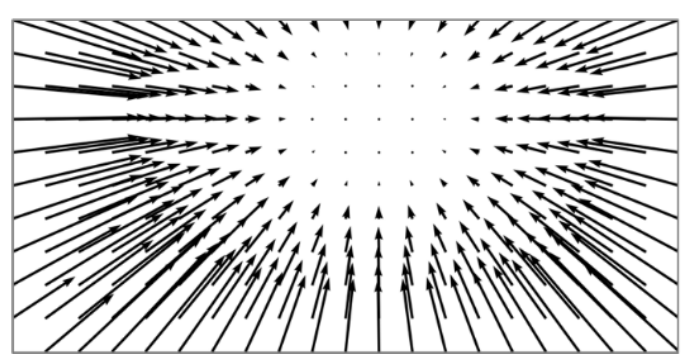

(a)

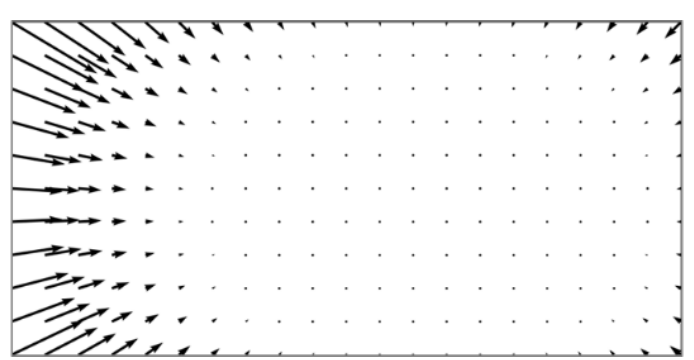

(b)

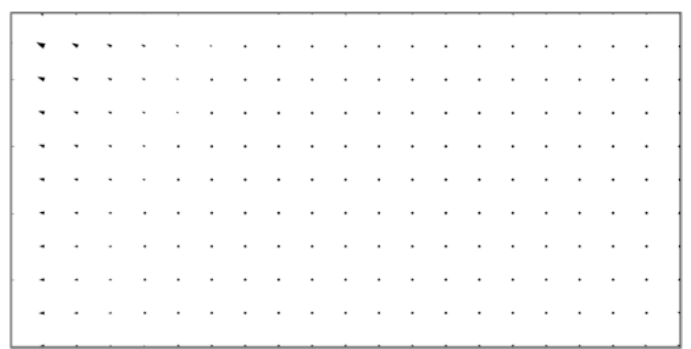

(d)

(c)

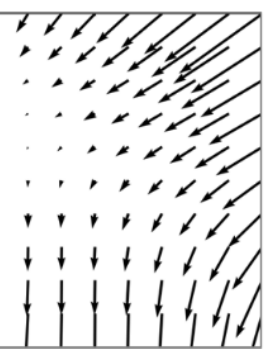

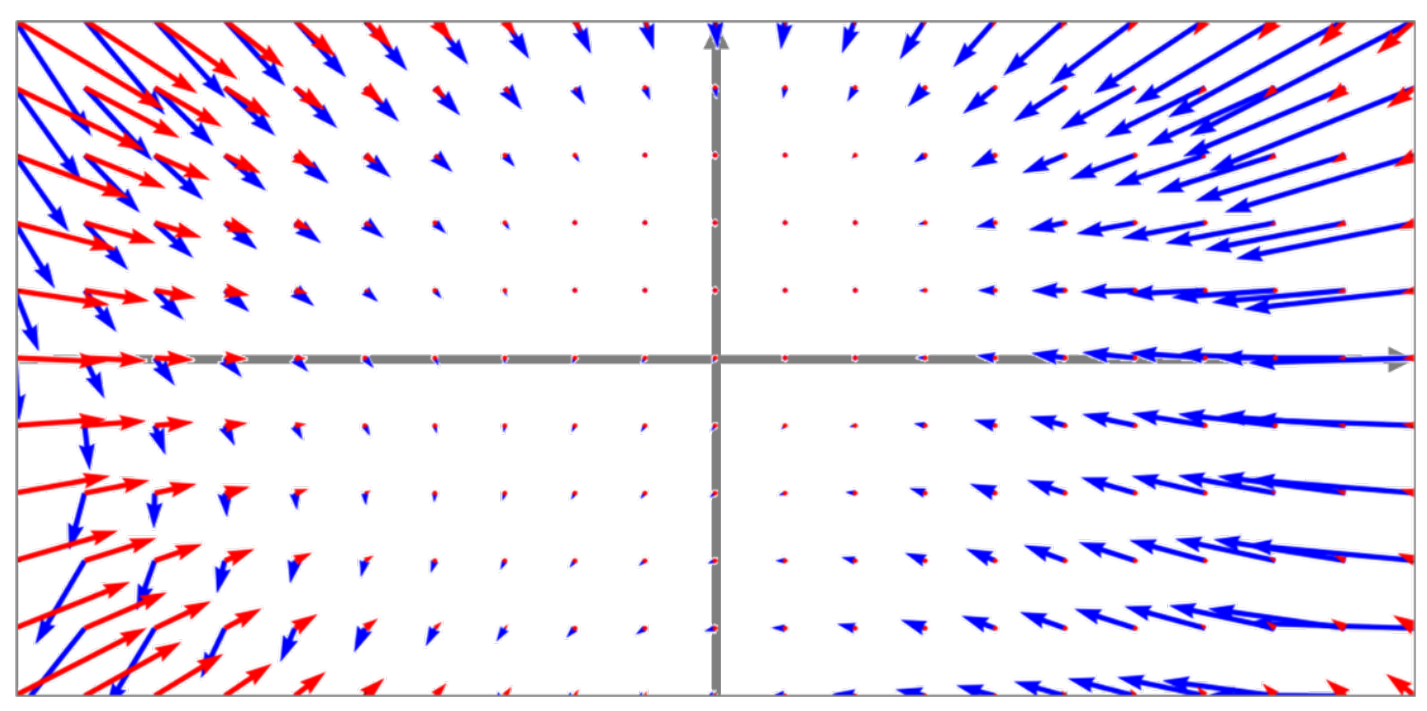

(e)

Figure 3.5: Distortion grids : (a) Radial distortion for smartphone camera. (b) Radial distortion for SLR camera. (c) Tangential distortion for smartphone camera. (d) Tangential distortion for SLR camera. (e) Total distortion for both smartphone camera (blue) and SLR camera (red). 


\begin{tabular}{ccccccc} 
Camera & $\begin{array}{c}\text { Mean } \\
\text { distance } \\
(\mathrm{mm})\end{array}$ & $\begin{array}{c}\text { Design } \\
\text { factor q }\end{array}$ & $\begin{array}{c}\text { Number } \\
\text { of images } \\
\mathrm{K}\end{array}$ & $\begin{array}{c}\text { Standard } \\
\text { deviation of } \\
\text { image mea- } \\
\text { surements } \\
\sigma_{\text {img }}(\mathrm{mm})\end{array}$ & $\begin{array}{c}\text { Theoretical } \\
\text { precision } \\
\sigma(m m)\end{array}$ & $\begin{array}{c}\text { Triangulation } \\
\text { accuracy } \\
\text { indicator } \\
\sigma / R\end{array}$ \\
\hline $\begin{array}{c}\text { Canon } \\
\text { EOS 1100D } \\
\text { Galaxy S7 } \\
\text { Edge }\end{array}$ & 310 & 0.6 & 24 & 0.016 & 0.019 & $1: 80000$ \\
& 256 & 0.6 & 63 & 0.02 & 0.013 & $1: 110000$ \\
& & & & & &
\end{tabular}

Table 3.3: Differences in estimated precision for both cameras.

the model created using the SLR camera. Some areas of notable difference can be distinguished; an important part of them being close to the edge of the cap or belonging to one of the markers that may have moved during the image acquisition. The figure also shows the histogram of the distances. The mean distance between models is 0.5 $\mathrm{mm}$, and the standard deviation $0.47 \mathrm{~mm}$. A small bias can be detected as the mean of the signed distances is $0.17 \mathrm{~mm}$ and the probability mass under zero is $41.6 \%$. Nevertheless, it is worth noticing that $86 \%$ of the distances between both models are below $1 \mathrm{~mm}$.

The differences between the ellipsoidal semiaxes (Table 3.4) are $1 \mathrm{~mm}$ for two of the semiaxes, B and C (shortest); the difference for semiaxis A (longest) is $0.6 \mathrm{~mm}$. The precision of each semiaxis is between 0.02 and $0.03 \mathrm{~mm}$.

\begin{tabular}{lccc} 
& Semiaxis A $(\mathrm{mm})$ & Semiaxis B $(\mathrm{mm})$ & Semiaxis C $(\mathrm{mm})$ \\
\hline Smartphone & $68.9 \pm 0.02$ & $75.4 \pm 0.03$ & $82.3 \pm 0.03$ \\
SLR camera & $68.3 \pm 0.02$ & $74.4 \pm 0.03$ & $81.3 \pm 0.03$
\end{tabular}

Table 3.4: Fitted ellipsoid parameters for both models.

\subsection{Discussion}

In medical applications, photography and photogrammetry are used for multiple purposes. However, their usage is not integrated as part of the consultation workflow and it is often related to high costs. One of these applications that we are envisioning is cranial deformation analysis, a condition that affects a great number of new-borns and 


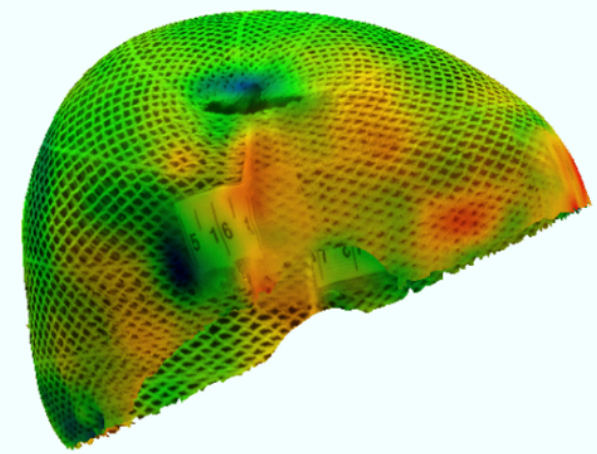

(a)

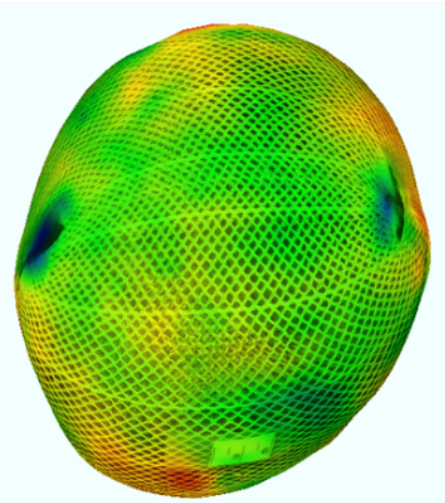

(c)

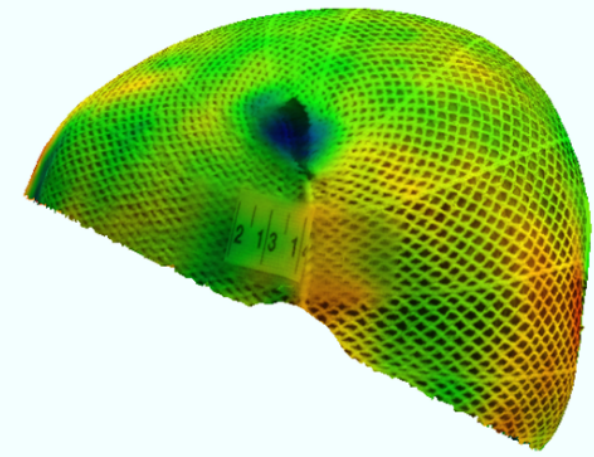

(b)

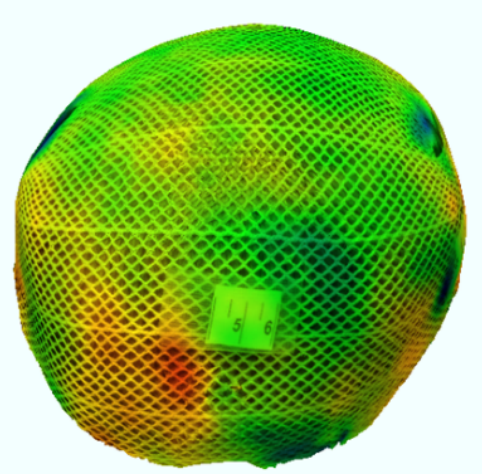

(d)

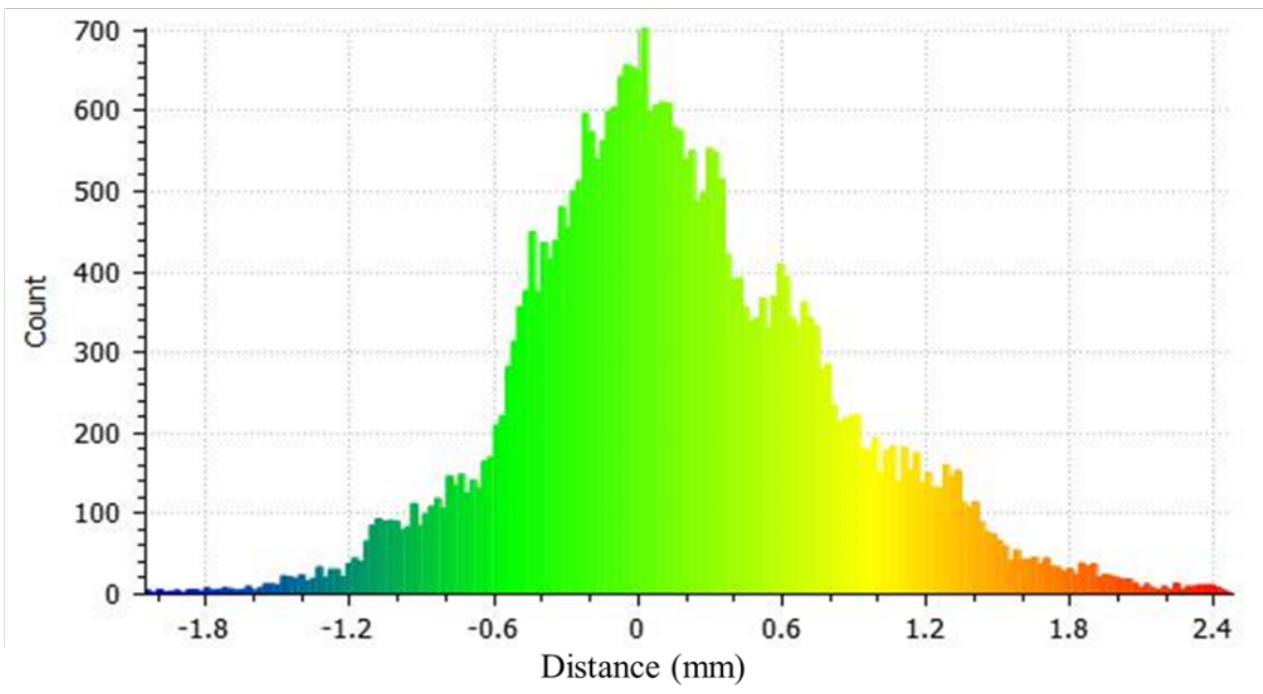

Figure 3.6: Model showing the differences between the models as hypsometric colours. Right view (a), left (b), top (c) and back (d). 


\section{AN SMARTPHONE-BASED APPLICATION}

that can vary from minor aesthetical problems (moderate positional plagiocephaly or brachycephaly) to serious diseases such as craniosynostosis (Pathmanaban et al, 2016). The extensive usage of smartphone cameras for this cranial deformation analysis is especially interesting as the method is highly flexible and has an affordable (low) cost. The use of a slow-motion video recorded with a smartphone is especially suitable for this specific application as it works well for uncontrolled moving targets and reduces considerably the time required for the image acquisition process. In addition, no special lighting is required due to the high sensitivity of smartphone cameras. The creation of 3D models of moving infants using SLR cameras is challenging. It was possible in this particular case because the infant was calmer than normal (although moving). The problems encountered when using SLR cameras were in the same trend even while taking pictures with a high-end digital camera, such as the Canon 1Ds Mark III with a Canon lens EF $35 \mathrm{~mm}$ lens. New-borns and children below 6-8 months are unconsciously moving either in the consultancy or in the hospital. In spite of their advantages, smartphone cameras - especially for slow-motion video- still have lower resolution than the $4 \mathrm{~K}$ video cameras and even the SLR cameras that are traditionally used for photogrammetric purposes. Smartphone cameras are also expected to have less stability on the interior orientation calibration, although this statement still needs to be confirmed from our side. Due to the demanding requirements in medical applications, it is vital to evaluate the accuracy and reliability of smartphone cameras for 3D modelling. In this study, we have compared two totally different cameras. In the first place, a professional, high-end SLR camera with a resolution of $4272 \times 2848$ pixels which requires the target to be still and needs a large amount of time to take the photographs. In the second place, a smartphone in slow-motion video mode recording, that despite its lower resolution (1280 x 738 pixels), allows users to take enough imagery data in shorter time slots. The camera self-calibration results show large errors, especially for the smartphone camera. The main limitation to obtain accurate calibration parameters is, undoubtedly, the geometry of the network. In this study, only a small area in the centre of the images was used. Moreover, not many accurate control points could be identified in the images. The identifiable points are concentrated on the measuring tape used as targets and the geometric distribution is far from ideal. For this reason, the calibration parameters need to be extrapolated providing very poor results. This 


\subsection{Discussion}

situation is a limitation given by the image acquisition situation. After this experimentation, a new data acquisition framework was figured out: the implementation of an independent on-the-job calibration framework using images acquired by the doctor prior to the video recording. The accuracy improvement expected with a calibration framework will be studied in the future in case higher accuracy is requested for the image-based 3D modelling solution. The estimates of the additional calibration parameters were notably worse for the smartphone-based camera. This can be explained by the lack of stability of the video camera, and it is well-known from literature (49). The image measurement standard error is $0.016 \mathrm{~mm}$ for the SLR camera, and slightly higher for the smartphone camera, $0.02 \mathrm{~mm}$. However, the video allows the acquisition of a larger quantity of images in shorter periods resulting in a better estimated precision for the smartphone model. The precision achieved is $0.013 \mathrm{~mm}$ for the smartphone model and $0.019 \mathrm{~mm}$ for the SLR camera model. These results show that the differences in the estimated precision between the two methodologies are not relevant. The mean Euclidean distance between the registered point clouds is $0.5 \mathrm{~mm}$. The mean of the signed distances is $0.17 \mathrm{~mm}$. With our large dataset (that included up to 34555 distances), this difference is significant and therefore it cannot be stated that models are the same. Nevertheless, the differences are small in the context of this application, as cranial deformation is usually measured using callipers that have a maximum accuracy of $1 \mathrm{~mm}$ in ideal conditions (rarely achieved in real clinical situations), without considering the landmarks identification error. Similar levels of accuracy are reported by other authors using high-cost special devices (Aldridge et al., 2005; Schaaf et al., 2010). The differences in the semiaxis of the fitted ellipsoids have a maximum of $1 \mathrm{~mm}$, which is within tolerance with the accuracy of the parameters. These two latter evaluations coming from the Euclidean distances as well as from the semiaxes comparison allow us to confirm that the theoretical precision estimations presented in Table 3.3 are too optimistic. Smartphone cameras in slow-motion mode are proven to be as suitable as SLR cameras for the creation of 3D models to evaluate cranial deformation in infants. In addition, smartphone slow-motion video reduces the time required to collect the images and produces even comparable results for analysing infants' head in movement. 


\subsection{Conclusion}

The use of smartphone cameras, especially those with slow motion video mode, were proved to be a suitable technique for the creation of 3D models intended to evaluate cranial deformation in infants at close ranges. Although SLR cameras provide a slightly better standard deviation for image measurements, the slow-motion video images taken with a smartphone allow users the acquisition of a higher number of well-focused images at lower resolution, and at closer ranges (ca. $30 \mathrm{~cm}$ ). Besides, neither additional lighting is requested to take neat images with low ISO numbers nor additional accessories such as tripod are required. After this research, it can be confirmed that smartphones can provide similar precision to SLR cameras, but with higher flexibility and ability to move around during data acquisition, at low-cost and without carrying external lighting equipment. Moreover, the concept of slow motion video works better than still images to achieve focused images for uncontrolled moving targets. The use of single 3D scanners is discarded for this application, as they are incompatible with infant's movement. 


\section{4}

\section{Adaptation to the best photogrammetric solution}

This chapter is based on the following paper: Barbero-García, I., Cabrelles, M., Lerma, J. L., \& Marqués-Mateu, Á. (2018). Smartphone-based close-range photogrammetric assessment of spherical objects. The Photogrammetric Record, 33(162), 283-299. https://doi.org/10.1111/phor.12243

\subsection{Introduction}

The performance of smartphone cameras has greatly increased over the past few years. Although their quality is still a limitation for obtaining highly accurate images, they have the great advantage of being totally portable and are almost always at hand. Currently, they offer a low-cost option for close-range photogrammetric applications, including the creation of 3D models for a wide range of purposes, including structural monitoring (66), geomorphology (67), creative industries (68) and medical applications (56, 69, 70), among others. Other low-cost non-metric cameras, such as webcams, have also been used for close-range modelling (71). Smartphone video (more specifically slow-motion video) is a useful tool for acquiring large numbers of images, suitable even for fast-moving objects. These images can be used for the creation of $3 \mathrm{D}$ models of moving objects (61). With an image acquisition speed of 240 frames per second (fps) of many smartphones (still far beyond the ultra-high-speed cameras that reach up to $2000 \mathrm{fps}$ ), the computational cost is the main limitation given the number of images to 


\section{ADAPTATION TO THE BEST PHOTOGRAMMETRIC SOLUTION}

be used for 3D modelling. Despite their advantages, smartphone cameras present high internal instability that hampers their correct calibration. This problem is common to all non-metric digital cameras (72), but is especially exacerbated when working with smartphones. The radiometric accuracy of smartphone cameras is lower than that of single-lens reflex (SLR) cameras but, despite their limitations, studies have concluded that these cameras can be used for photogrammetric tasks with a required accuracy of 1:10 000 (73). The development of useful tools, which could allow non-expert users to obtain accurate 3D models for different purposes, requires a high degree of automation (74). However, most of the available automatic low-cost solutions provide low repeatability and reliability (75). The development of fully automatic and reliable solutions for specific applications requires extensive knowledge of the factors affecting the quality of the 3D models created using smartphones or other similar imaging devices, such as tablets. The most important factors include the determination of the ideal geometric network, the selection of the best video frames and their optimal number, as well as the accuracy requirements for camera calibration. These parameters can vary greatly depending on the characteristics and limitations of the image acquisition process for a specific application (such as moving or static target feature, camera-to-object distance, without forgetting the lighting conditions) and the required accuracy of the final 3D model. The traditional working pipeline in photogrammetry includes a low number of images and the manual identification of tie points. In contrast, the automatic processes that are common nowadays require a large number of images and, therefore, short baselines. The high overlap allows the use of feature-detection algorithms such as the scaleinvariant feature transform (SIFT) (76) and speeded-up robust features (SURF)(77), so that tie points are detected automatically. The high speed of image acquisition also points to hyper-redundancy as a way to improve accuracy and compensate for the lack of internal stability. Some authors have studied the influence of hyper-redundancy and suggest using it as a tool to improve accuracy when the additional work to extract a large number of images is not an issue (78). Current technologies, such as video, provide huge quantities of data. Therefore, the accurate determination of the number of images required for modelling, and the consequent filtering out of redundant images, has also become a necessity (79). Another common subject of study in photogrammetry is the possibility of accurate self-calibration, especially for digital cameras with poor internal stability. Although self-calibration, conducted simultaneously with the 3D-modelling 
process, is considered a powerful tool, a separate calibration process using a setup that ensures good image geometry is still recommended in many cases. A primary reason behind this is that the optimal network for $3 \mathrm{D}$ reconstruction is usually not the best for camera calibration (80). Lastly, it is important to take into account the variety of capabilities and characteristics of different smartphone cameras. Resolution and maximum frame rate vary greatly between devices, providing very different results. This study assesses the different settings that can affect the creation of accurate smartphone $3 \mathrm{D}$ models for spherical objects in close-range applications. Spherical objects were selected as the final goal is to find the optimal setup for a specific application, namely the creation of 3D models for cranial deformation analysis in infants. In this application, the object to be modelled (the infant's head shape) is usually in movement and image acquisition is carried out by a doctor in a limited time frame (61). However, as the real photogrammetric data-acquisition conditions are hard to assess and replicate, this study deals with a sphere whose shape emulates an infant's head. Therefore, it is possible to take as many shots as necessary in order to replicate the different conditions that can be encountered in real-life projects, usually in either a hospital or a clinic. A wide range of conditions was tested to determine the ideal network and setup. Due to the type of application, the setup was a simplification of real clinical conditions. Different numbers of image sets (19 to 95) were used to generate different models in order to evaluate the effect of hyper-redundancy. In addition, the influence of both (separate) camera calibration and (integral) self-calibration were tested, as both were performed for each of the network designs. The use of well-defined automatic coded markers as a tool to improve reliability and accuracy $(\underline{81}, 82)$ was also evaluated. Two different smartphones, with different camera characteristics, were tested. Reference data were obtained using a calibrated high-end SLR camera. The coordinates of a set of control points were calculated for each of the created models. The distances between each pair of coordinates were calculated too; the differences from the reference data were then obtained. The completeness of the models was also evaluated. The results of the study will be useful for the automation of the process and the creation of clear guidelines that will allow users (namely doctors) to successfully carry out the image acquisition. 


\section{ADAPTATION TO THE BEST PHOTOGRAMMETRIC SOLUTION}

\subsection{Methodology}

The evaluation of different parameters for 3D modelling, including network design, number of images, use of targets and calibration methodology, will drive the selection of the best methodology for 3D modelling close-range spherical objects, at an approximate camera-to-object distance of $30 \mathrm{~cm}$ with low imaging texture. Two smartphones were tested to assess the methodology: a Samsung Galaxy S7 Edge and a Samsung Galaxy Trend. The former can be considered a high-end smartphone; the latter a more conventional and cheaper smartphone. A high-end SLR camera, a Canon EOS-1Ds Mark III, was used to determine highly accurate coordinates of the discrete targeted points after self-calibration. These targeted points acted as control points and were used as reference data for 3D assessment. The first step of the photogrammetric data processing started with the geometric calibration of each camera, to determine both the interior and the exterior orientation parameters, as well as the additional parameters. The setup consisted of: (1) one horizontal and two vertical panels (in an L-shaped configuration), all of them with several targets already included on them, as well as additional coded targets); (2) the sphere to be modelled; and (3) a calibrated ruler. The data was processed using the in-house photogrammetric software FOTOGIFLE (83, 84), which allows users to determine the quality of all the estimates (interior and exterior orientation parameters, and object coordinates). After the geometric calibration, one video of the object to be modelled was taken with each smartphone. Next, the Agisoft PhotoScan software was used to create several models for each video, varying the number of images, the presence of markers (targets) and the geometric calibration of the cameras (Fig. 4.1).

\subsubsection{Calibration}

The camera calibration was carried out using a setup of panels with targets, with the object to be modelled placed inside the framework defined by the panels (Fig. 4.2) . Although the inclusion of the object was not necessary for calibration purposes, it simplified the processing as the images for the self-calibration of the SLR camera were also used to create the reference 3D model. For the Canon SLR camera calibration, a set of images were taken using a tripod and standard room illumination. The selfcalibration setup included a set of convergent images, some of them rotated by $90^{\circ}$, 


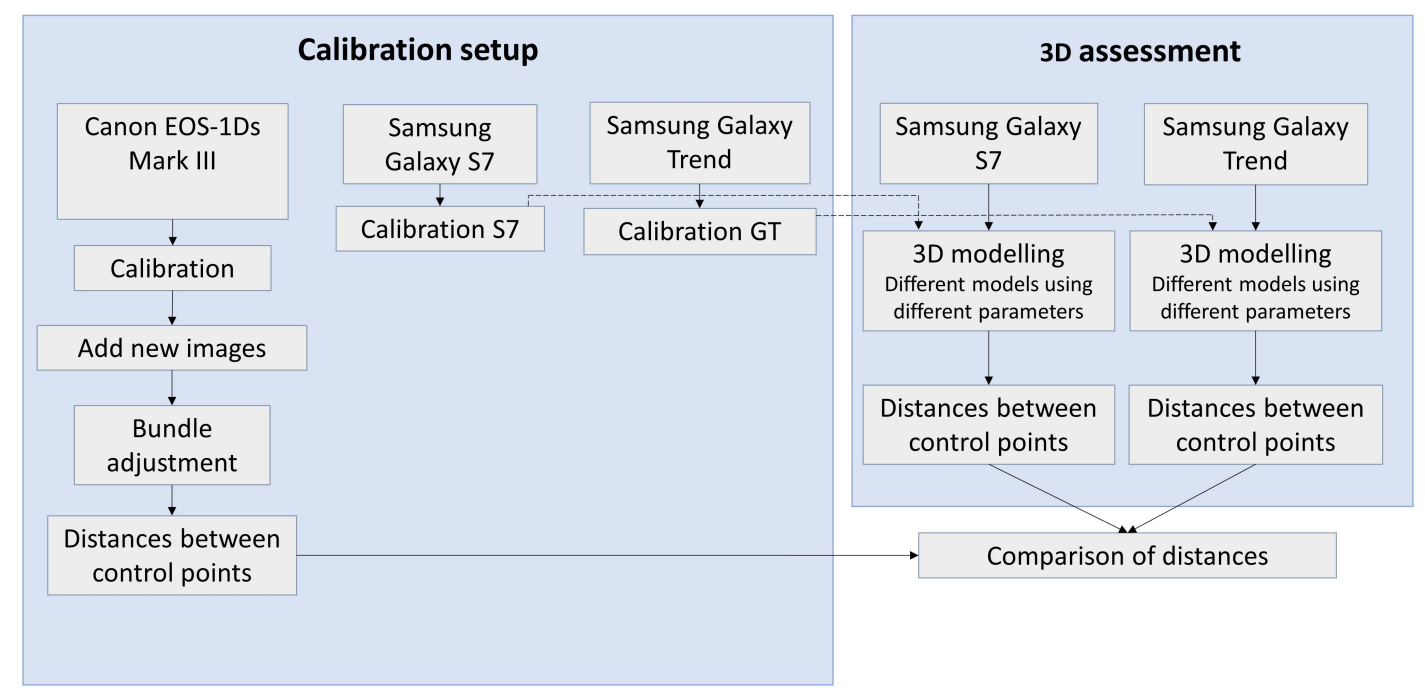

Figure 4.1: Methodology workflow for calibration and 3D assessment

good intersection angles of rays from the object points to the camera positions, and a sufficient number of targets well spread across the image format. This geometry was chosen to assure a good calibration, as stated by Fryer (85) and Remondino and Fraser (80). Finally, the images were calibrated using FOTOGIFLE with up to 10 additional parameters as proposed by Fraser (49). A total of 89 circular and coded targets were used for the calibration, achieving an average calibration error of 0.26 pixels and a maximum error of 1 pixel. The resulting targeted points coordinates were set as fixed coordinates to calibrate both smartphones afterwards. To calibrate the smartphone cameras, a video was recorded with each device. The conditions (distance, resolution, lighting...) used for this video calibration were the same as used later for recording and carrying out the subsequent 3D modelling. A set of 11 frames from each video were manually selected to satisfy the best possible geometry. Most of the required targets were manually identified in each image set, due to the lack of automatic recognition by PhotoScan.

\subsubsection{Three-dimensional model setup}

A sphere $18 \mathrm{~cm}$ in diameter was used for the tests. This object tries to emulate, in a simplified form, an infant's head modelled for cranial deformation analysis. The clinical conditions were also replicated and thus neither special lighting nor a tripod 


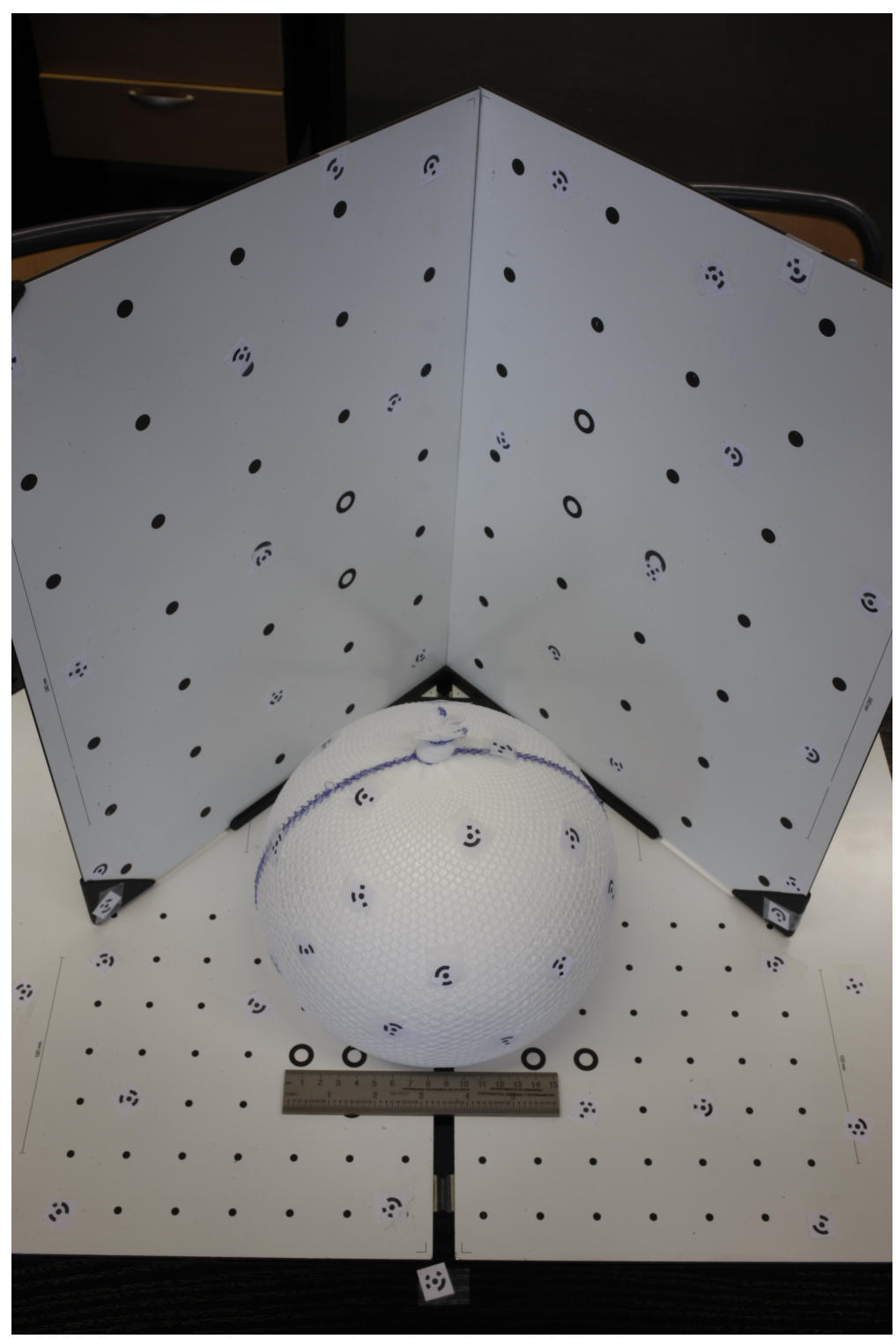

Figure 4.2: Setup used for camera calibration that includes three orthogonal calibration panels, the sphere to be modelled and a calibrated ruler. Additional coded targets were incorporated to strengthen the geometry. 
were used. To further imitate clinical conditions and facilitate the 3D modelling by adding some texture, a fitted cap, similar to those used on the patients, was placed on the sphere. A total of 24 coded targets, with an approximate diameter of $0.5 \mathrm{~cm}$, were placed on the panels and sphere. These coded targets were recognised automatically by the PhotoScan software as markers; they were also used as control points for accuracy calculations. A calibrated ruler was placed next to the sphere to allow the scaling of the reference dataset (Fig. 4.3).

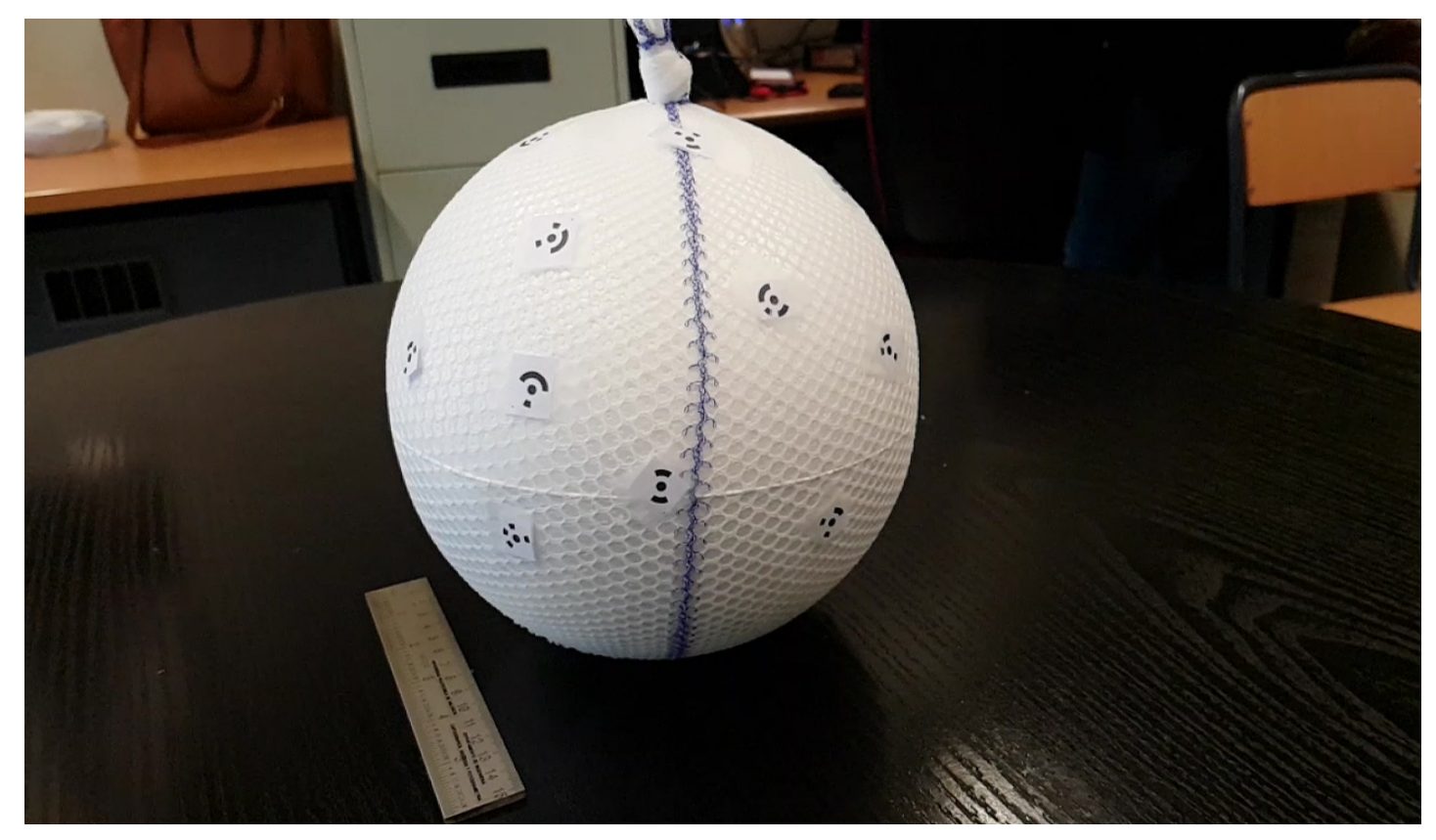

Figure 4.3: Three-dimensional model setup

\subsubsection{Reference data}

A high-accuracy photogrammetric solution was chosen as the best methodology to obtain accurate coordinates of the targets. The Canon EOS-1Ds Mark III SLR was used with a pixel count of $5616 \times 3744$, a focal length of $35 \mathrm{~mm}$ and ISO speed of 100 (Table 4.1). A total of 40 images were taken covering the sphere and targets around it to assure the best possible accuracy. A tripod was employed to achieve neat pictures. The setup was the same as used for calibration purposes, and the images used for the calibration were also included. 29 further images were added, covering the back-side of the sphere after removing the vertical panels used for calibration. The coordinated 


\section{ADAPTATION TO THE BEST PHOTOGRAMMETRIC SOLUTION}

control points (coded targets) were computed with the software FOTOGIFLE, using a bundle adjustment and the camera calibration parameters obtained in a previous step during the camera calibration (Fig. 4.1). Sub-millimetre precision was obtained for every control point.

\subsubsection{Image acquisition}

A video was recorded with each smartphone. The maximum frame rate for each device was chosen (Table 4.1). A slow-motion video with a 1280x720 pixel count and $239 \mathrm{fps}$ was obtained using the Samsung Galaxy S7 smartphone. No slow-motion functionality was available on the Samsung Galaxy Trend Plus. Therefore, a video with a similar pixel count and $30 \mathrm{fps}$ was obtained. The length of both videos was under 1 minute. The specifications of the cameras can be consulted in Table 4.1, although not all the parameters are provided by the manufacturer for the Samsung Galaxy Trend.

\begin{tabular}{lccccc} 
Camera & $\begin{array}{c}\text { Focal } \\
\text { length } \\
(\mathrm{mm})\end{array}$ & $\begin{array}{c}\text { Maximum } \\
\text { frame rate } \\
(\mathrm{fps})\end{array}$ & $\begin{array}{c}\text { Effective } \\
\text { format size } \\
(\mathrm{mm})\end{array}$ & Pixel count (pixels) & $\begin{array}{c}\text { Pixel } \\
\text { pitch } \\
(\mathrm{mm})\end{array}$ \\
\hline $\begin{array}{l}\text { Canon } \\
\text { EOS-1Ds }\end{array}$ & 35 & & $35.94 \times 23.96$ & Still: $\mathbf{5 6 1 6 \times 3 7 4 4}$ & 0.0064 \\
$\begin{array}{l}\text { Mark III } \\
\text { Samsung }\end{array}$ & 4.17 & 239 & $5.37 \times 3.02$ & $\begin{array}{c}\text { Still: } 3840 \times 2160 \\
\text { Video: } \mathbf{1 2 8 0 \times 7 2 0}\end{array}$ & 0.0014 \\
$\begin{array}{l}\text { Galaxy S7 } \\
\text { Samsung }\end{array}$ & & & & Still: $2048 \times 1536$ & 0.0042 \\
$\begin{array}{l}\text { Galaxy } \\
\text { Trend Plus }\end{array}$ & Unknown & 30 & Unknown & $\begin{array}{c}\text { Video: } \mathbf{1 2 8 0 \times 7 3 8} \\
\text { Unknown }\end{array}$ &
\end{tabular}

Table 4.1: Camera parameters. The mode actually used is highlighted in bold.

\subsubsection{Network design}

The determination of the optimal network design is especially important for the application of the methodology in real working environments. Under clinical conditions, simplicity and speed of data acquisition are vital to present a realistic and useful methodology. Two different camera configurations were tested:

1. The first network consisted of a single ring of nearly horizontal convergent images taken around the object (Fig. 4.4 a). Two models were created: (i) using 26 
images; and (ii) using 52 images.

2. The second network consisted of two sets of rings taken at different heights around the object, together with a third ring of zenithal (nadir) images (Fig. $4.4 \mathrm{~b}$ ). Four models were tested: (i) 19 images ( 9 at low height +9 at medium height +1 at the top); (ii) 26 images $(16+9+1)$; (iii) 52 images $(30+20+2)$; and (iv) 95 images $(55+25+15)$.

The model with 19 images (2(i) above) was considered the generic network design, similar to the one presented by Kraus (60) for similar shapes, as it is the one where the optimal multi-ray intersection is obtained with as few images as possible. Therefore, the other networks should be considered redundant. The set of images for each model was manually selected to obtain the optimal geometry. In order to evaluate the effect of the calibration, every model was calculated three times using different geometric camera calibration methodologies:

1. Self-calibration from PhotoScan.

2. Fixed interior (inner) calibration from FOTOGIFLE on an optimal network, and image orientation in PhotoScan.

3. Self-calibration and image orientation in PhotoScan using interior calibration parameters from FOTOGIFLE as pre-calibration data.

For methodology (3), the calibration parameters obtained using FOTOGIFLE were imported into PhotoScan in Australis format (86) and then transformed by PhotoScan into its own format. This process was necessary as the calibration parameters are defined differently in each software (87). Although different workflows were followed to determine the interior orientation parameters, the external orientation was always carried out using PhotoScan. To evaluate the effect of the presence of coded targets, every model was calculated both with and without them. The whole process consisted, therefore, of a total of 36 3D models (6 networks; 3 calibrations; 2 target types) for each smartphone. Every model was scaled using five ground control points (GCPs) whose coordinates were extracted from the reference data. 


\section{ADAPTATION TO THE BEST PHOTOGRAMMETRIC SOLUTION}

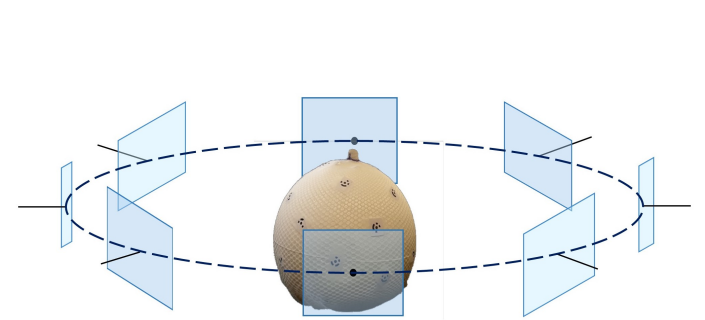

(a)

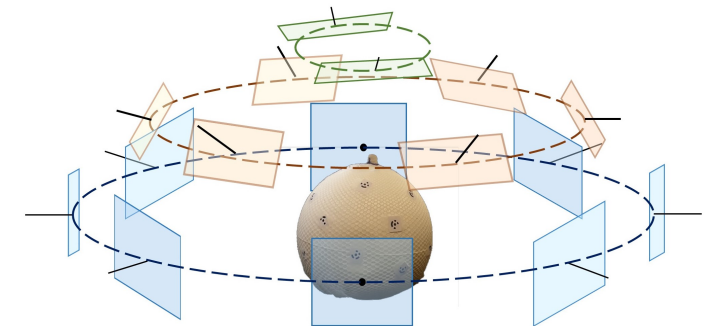

(b)

Figure 4.4: Camera network geometry. (a) Single ring of nearly horizontal images at a constant height around the object. (b) Three rings of images at different altitudes and employing horizontal, oblique and zenithal (nadir) attitudes.

\subsubsection{Model creation}

In the first place, the images were masked to exclude the background. When using images of moving objects in live environments, the background changes its position in relation to the object. Therefore, the background must be excluded to allow the creation of the 3D model. In this particular case, using the background would allow the identification of points outside the object and the results would not represent the true condition that would be achieved on site. After masking, some targets were manually identified, as PhotoScan was unable to detect all of them automatically. Lastly, the model was created using the conventional software pipeline: (1) image orientation; (2) dense matching; and (3) meshing. Finally, every model was scaled using the five GCPs whose coordinates were provided by the reference model. An additional step was also undertaken: model texturing for visualisation purposes.

\subsubsection{Assessment}

The assessment was carried out considering two parameters: (1) the accuracy of the targets coordinates; and (2) the completeness of the model. The accuracy of the coordinates was evaluated by comparing the distances between targets. For each model, a total of 171 distances between all possible pairs of targets were calculated. Later, the distances were compared to those of the reference dataset. This process, as presented by Luhmann and Wendt (88), does not need registration of the models and, consequently, registration errors are avoided. The completeness of the models was checked visually. The models were classified as complete when the whole sphere was correctly 
represented, and as not complete when holes were apparent in a model (Fig. 4.5). In addition, a further category was considered for incomplete 3D models that presented only small imperfections on the top; these are termed 'bare' models. This latter case is due to the lack of zenithal images during the single ring data acquisition (Fig. 4.4 a).

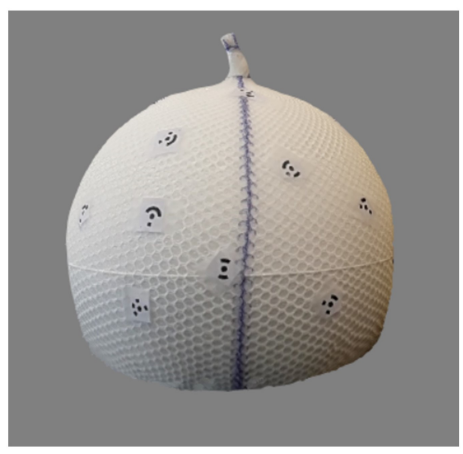

(a)

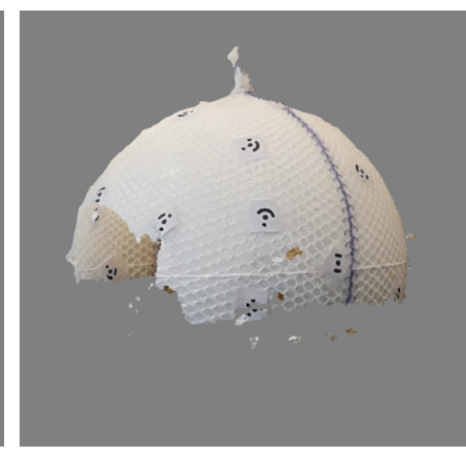

(b)

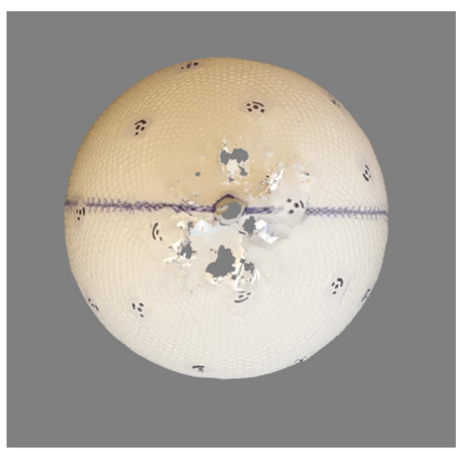

(c)

Figure 4.5: Examples of: (a) a complete three-dimensional model; (b), incomplete model; and (c) a model with small imperfections (bare).

\subsection{Results}

\subsubsection{Calibration}

Calibration results are presented in Table 4.2. For both smartphone cameras, in addition to the elementary interior orientation parameters $(\mathrm{x} 0, \mathrm{y} 0, \mathrm{f})$, two radial lens distortion parameters were requested $(\mathrm{k} 1, \mathrm{k} 2)$, as well as the first decentring lens distortion parameter (p1) and the in-plane differential scaling between the horizontal and the vertical pixel spacing (b1). Every parameter was checked for statistical determinability and those below 99\% were removed. In total, the Samsung Galaxy S7 required seven additional parameters while Samsung Galaxy Trend Plus required nine parameters (two more than the other smartphone: p2 - the second decentring lens distortion parameter; and b2 - non-orthogonality between the $\mathrm{x}$ and $\mathrm{y}$ axes). The mean of the residuals of the bundle adjustment obtained using FOTOGIFLE for the Samsung Galaxy Trend smartphone was below 1.39 pixels, with an average of 0.36 pixels. For the Samsung Galaxy S7 smartphone calibration, the residuals were 1 pixel higher, with an average of 1.36 pixels. These residuals could be reduced only by performing an independent 


\section{ADAPTATION TO THE BEST PHOTOGRAMMETRIC SOLUTION}

calibration for each photograph. However, this option was discarded as independent calibration parameters, obtained using an ideal calibration network, would not be applicable for the rear-side 3D modelling. Worth noting is that the standard deviation of the additional calibration parameters with the Samsung Galaxy Trend was always better than on the Galaxy S7 counterpart. The average intersection angle was $41.9^{\circ}$ for the Samsung Galaxy S7 and $26.3^{\circ}$ for the Samsung Galaxy Trend. The calibration distortion patterns (Fig. 4.6) do not show large differences between the two devices.

\begin{tabular}{lcccc} 
& \multicolumn{2}{c}{ Galaxy S7 } & \multicolumn{2}{c}{ Trend Plus } \\
\hline & Mean & $\sigma$ & Mean & $\sigma$ \\
\hline x0 & 16.68 & 0.47 & 15.65 & 0.24 \\
y0 & -24.16 & 0.35 & 32.61 & 0.27 \\
$\mathrm{f}$ & -1029.28 & 0.44 & -1292.32 & 0.22 \\
k1 & $-1.30-07$ & $2.09-09$ & $-1.38-0$ & $6.97-10$ \\
k2 & $2.50-13$ & $3.70-15$ & $2.20-13$ & $1.36-15$ \\
k3 & 0 & 0 & 0 & 0 \\
p1 & $7.56-07$ & $1.60-07$ & $1.06-06$ & $4.92-08$ \\
p2 & 0 & 0 & $-9.48-07$ & $6.51-08$ \\
b1 & 0.0235 & $1.40-04$ & 0.0255 & $4.71-05$ \\
b2 & 0 & 0 & 0.0017 & $4.52-05$ \\
Mean error in x & \multicolumn{3}{c}{1.36} & \multicolumn{2}{c}{0.34} \\
Mean error in y & \multicolumn{2}{c}{1.13} & \multicolumn{2}{c}{0.36} \\
\hline
\end{tabular}

Table 4.2: Additional calibration parameters and standard deviation $(\sigma)$ obtained using FOTOGIFLE.

\subsubsection{Three-dimensional models}

A total of 36 models were created for each device, so 72 models in total. The coordinates of the coded targets in each model were obtained by combining automatic and manual procedures whenever the fully-automatic solution did not work. Later, the distances between every pair of markers were calculated and compared to those of the reference dataset derived from the high-end SLR digital camera. The mean difference in distance and the 65 th percentile are shown in Table 4.3 . This table also specifies if the model was complete and acceptable (in green); incomplete (in yellow); or the additional 'bare' category (in blue) for those models that were almost correct 


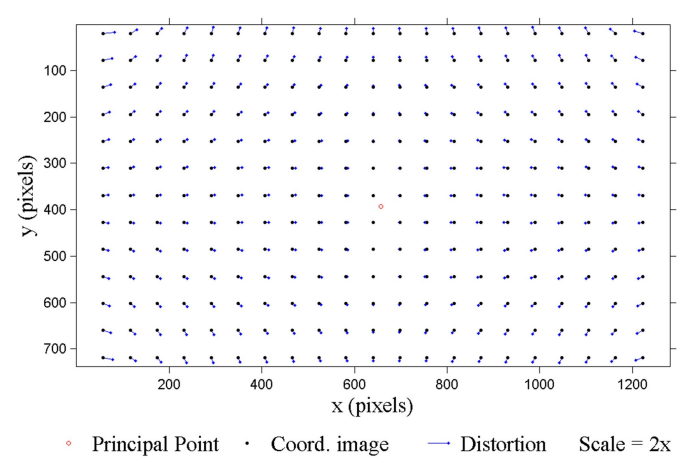

(a)

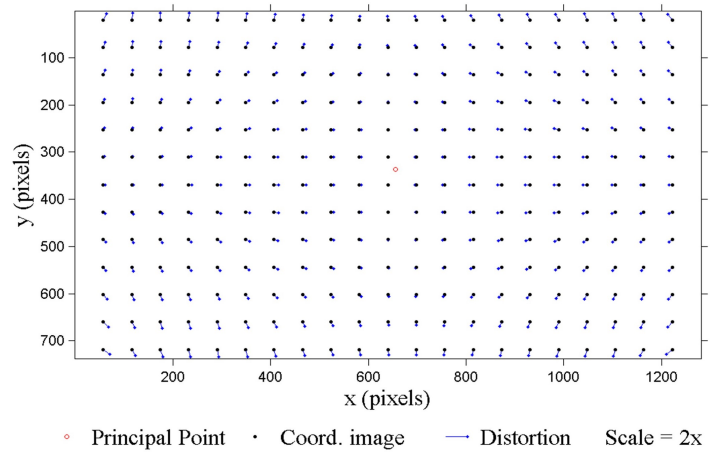

(b)

Figure 4.6: Calibration distortion patterns for (a) the Samsung Galaxy S7 and (b) the Samsung Galaxy Trend.

but presented small imperfections, mainly in the upper area of the object. This bare category was necessary as some network designs, that achieved high accuracies, failed to produce useful 3D models without any additional processing. Therefore, this bare category highlighted those 3D models demanding additional photogrammetric and/or editing processing. In addition, 3D models that were not achieved after the automatic photogrammetric workflow are highlighted in orange.

For the Samsung Galaxy S7 and the simple network design with a single ring of nearly horizontal images, models were nearly complete but presented small imperfections, mostly in the upper part which was not correctly covered by the images. However, the calculated distance error was acceptable, being below $1 \mathrm{~mm}$ for $65 \%$ of the points in every case. The results were almost independent of the type of calibration and the presence of targets. For the second network design with three rings of imagery, no models were obtained with 19 images and some problems appeared in areas without targets using 26 and 52 images. In the last case which used 95 images, all models were complete; the error was below $1 \mathrm{~mm}$ in every case and under $0.5 \mathrm{~mm}$ whenever targets were used. Despite the differences registered in the completeness of the various models, no significant improvement in the accuracy of the models was achieved by increasing the number of images. For the Samsung Galaxy Trend smartphone, with a significantly less powerful camera, no complete models could be obtained with fewer than 95 images. On the one hand, the best result was obtained in the 95-image network, using targets and with no fixed independent calibration, yielding an average error of $0.2 \mathrm{~mm}$. On 


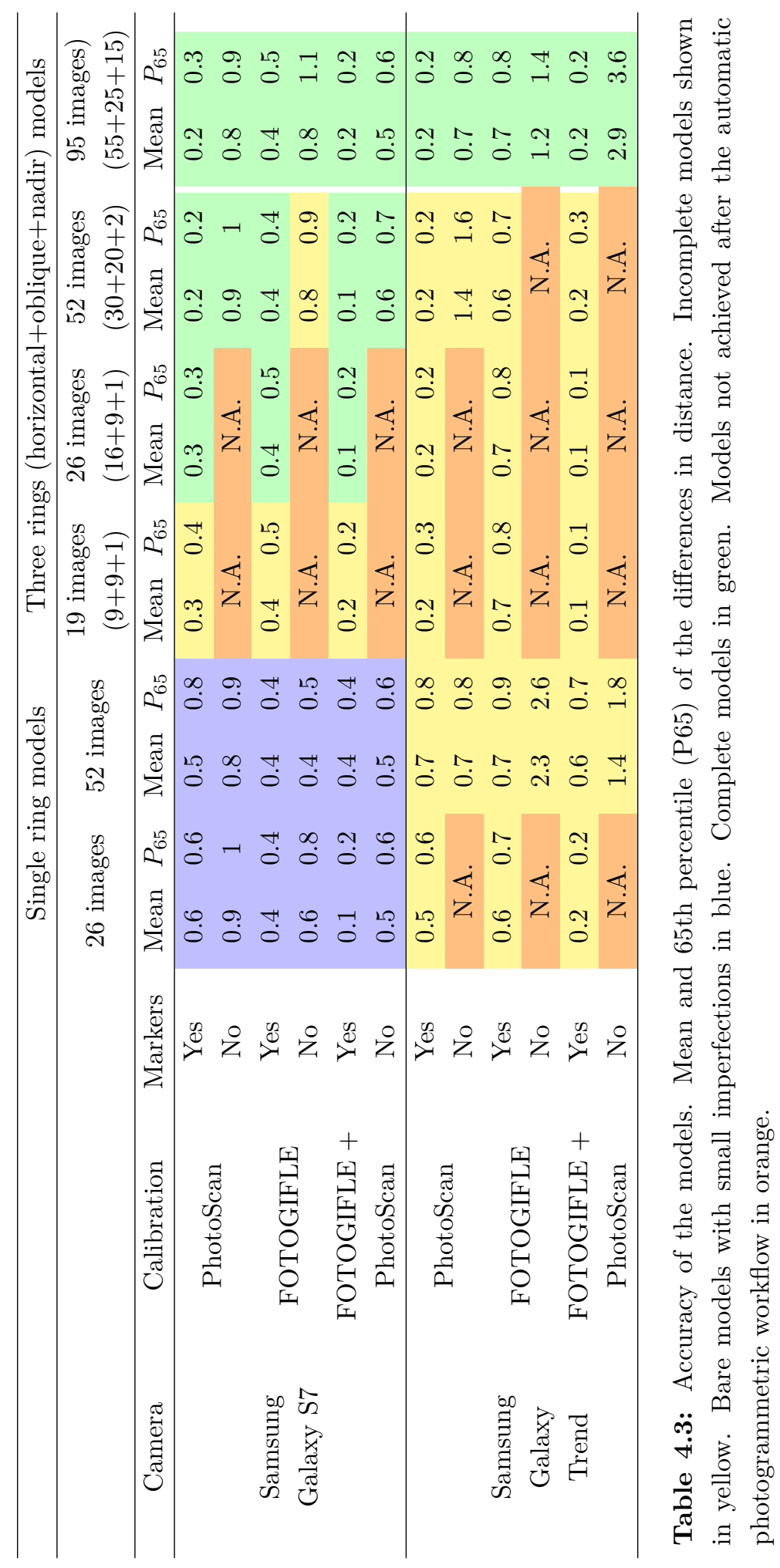


the other hand, the error increases without targets, the lowest mean being $0.7 \mathrm{~mm}$. The influence of the individual parameters is presented in Figure 4.6. The usage of coded targets is the most important (key) factor to improve accuracy (Fig. 4.7 a). The models created without targets have a maximum error of $1.4 \mathrm{~mm}$ while the models with targets have an error below $0.7 \mathrm{~mm}$ in every case. Targets also helped to increase reliability. The self-calibration and the fixed FOTOGIFLE calibration provided similar accuracy (Fig. 4.7), while the non-fixed calibration, using first the calibration from FOTOGIFLE and afterwards slightly improvements from PhotoScan, provided a significant improvement. This type of calibration also improved reliability slightly. The two different devices showed very different reliability values. The Samsung Galaxy S7 provided correct models in $40 \%$ of the cases while only $10 \%$ of the models were correct when using the Samsung Galaxy Trend. The accuracy was also significantly better on the S7 device. The two network geometries used presented very different results for both accuracy and reliability. The one-ring network geometry provided slightly smaller errors in coordinates. Nevertheless, the reliability of the results was much better for the three-ring geometry.

Due to the high influence of the coded targets in the final 3D modelling assessment, it is necessary to analyse the results of the different networks and the number of images, with and without targets, to obtain meaningful results (Fig. 4.8). The accuracy was better than $1 \mathrm{~mm}$ for every network geometry and number of images whenever targets were included. However, the reliability was really affected by both the geometry and the number of images; only the models with 95 images were correct in every case.

\subsection{Discussion}

Smartphone cameras in slow-motion video mode have proven to be a useful tool for the quickly obtaining images that can be used for various close-range photogrammetric applications. This study has evaluated the possibilities of two smartphone cameras for close-range photogrammetry (at approximately a $30 \mathrm{~cm}$ camera-to-object distance) and 3D modelling of a small spherical object with low image texture. In particular, the effects of calibration, hyper-redundancy and the presence of well-defined targets have been evaluated. This has been achieved by keeping in mind a particular application: the 


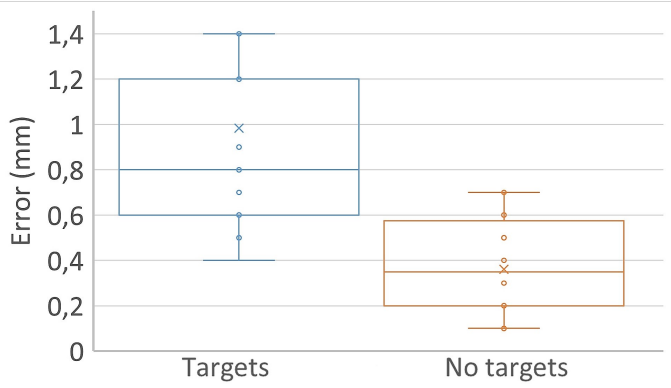

(a)

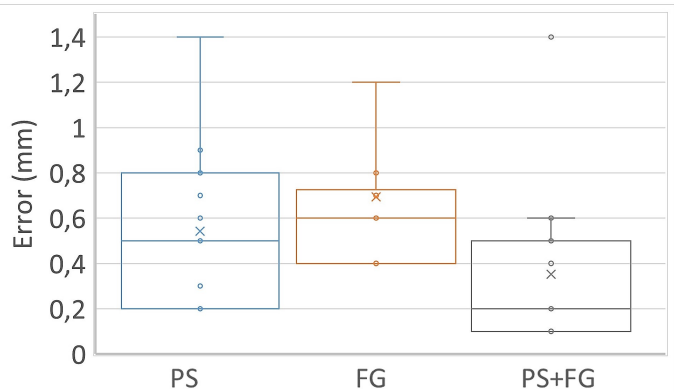

(c)

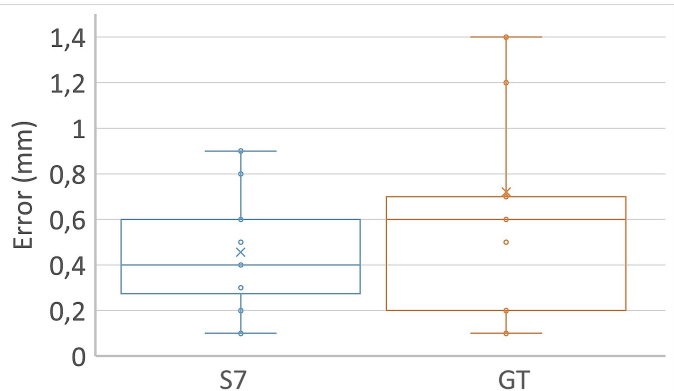

(e)

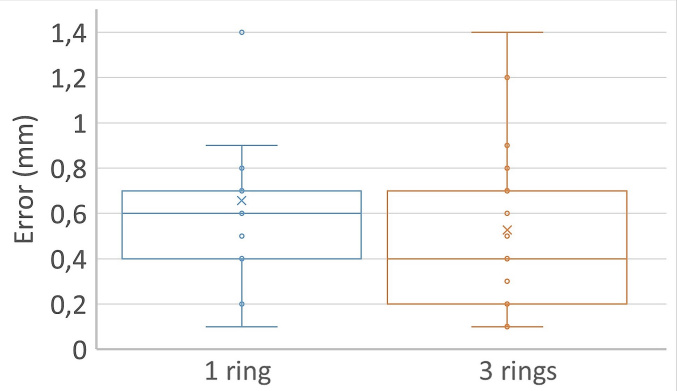

(g)

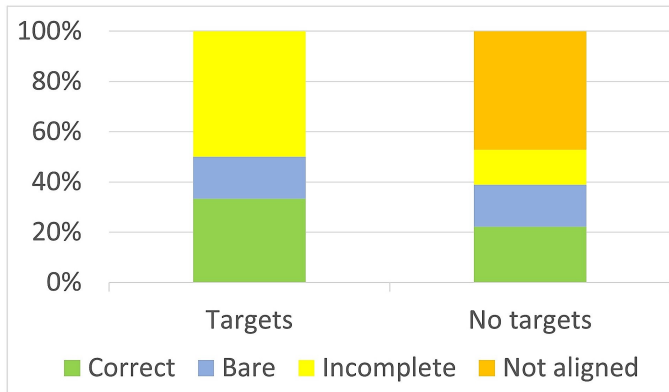

(b)

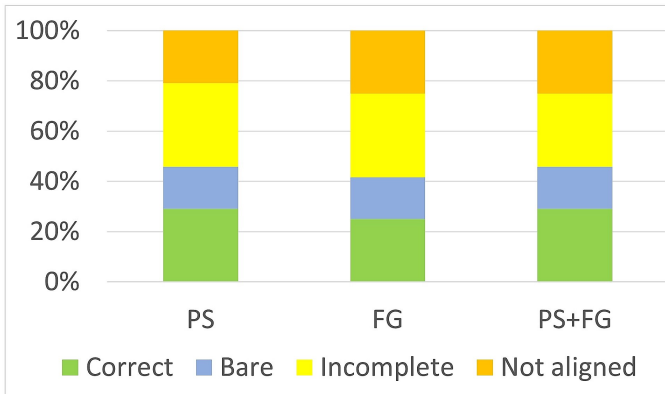

(d)

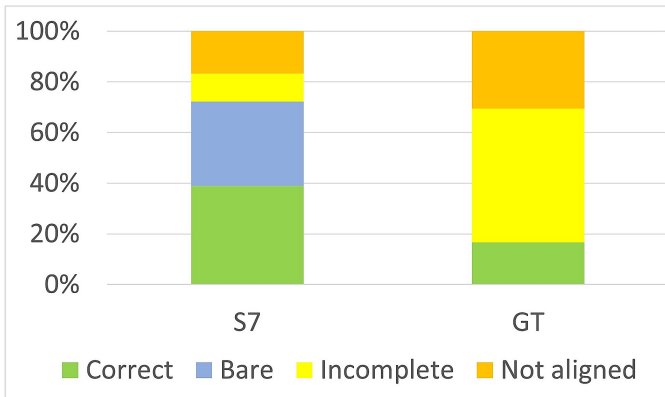

(f)

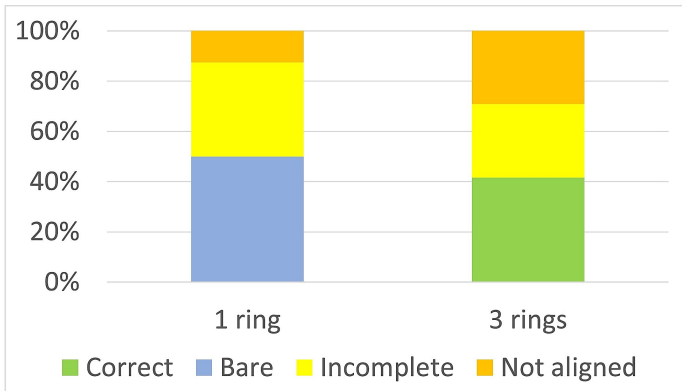

(h)

Figure 4.7: Box-and-whisker plots showing the accuracy of the models (using the mean error for each one) grouped by: (a) the presence/absence of targets: (c) calibration approach; (e) device; and (e) camera network geometry. Reliability of the models for the same set of parameters (b, d, f, h). 


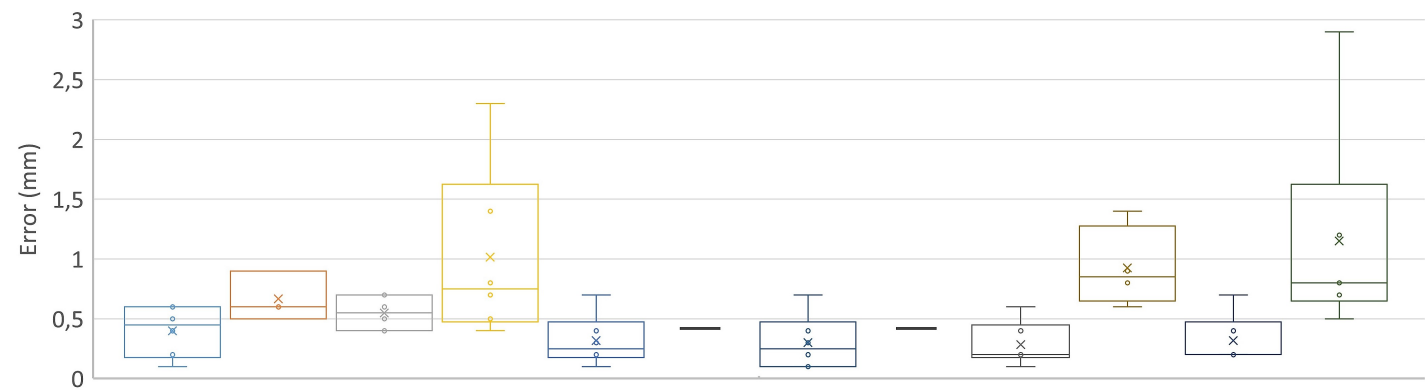

26 images 26 images 52 images 52 images 19 images 19 images 26 images 26 images 52 images 52 images 95 images 95 images with targets without with targets without $(9+9+1) \quad(9+9+1) \quad(16+9+1) \quad(16+9+1) \quad(30+20+2)(30+20+2)(55+25+15)(55+25+15)$ targets targets with targets without with targets without with targets without with targets without targets targets targets

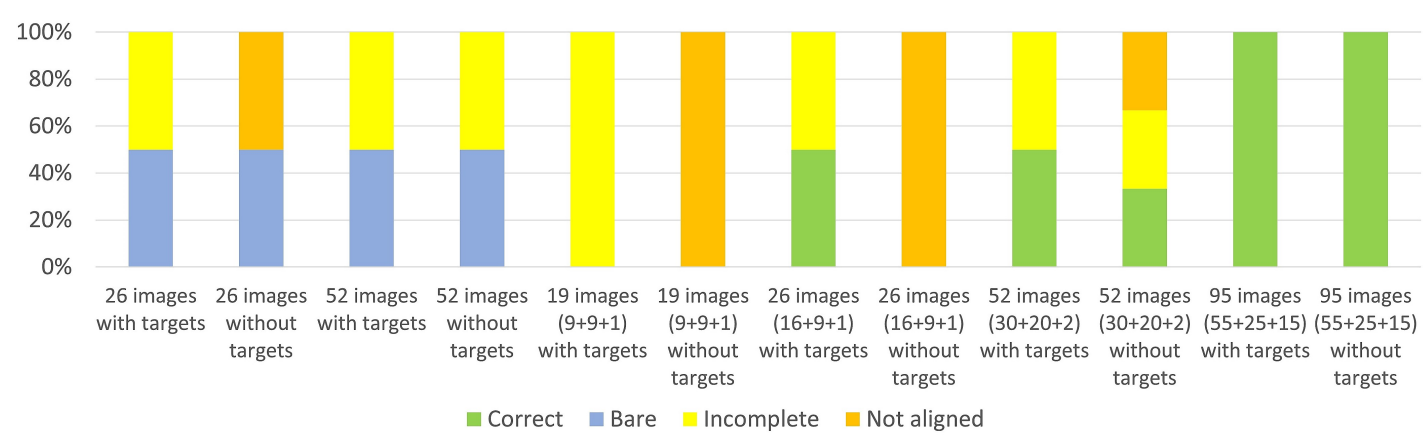

Figure 4.8: Accuracy (top) and reliability (bottom) for the different network geometries based on the number of images, with and without targets. 


\section{ADAPTATION TO THE BEST PHOTOGRAMMETRIC SOLUTION}

modelling of infants' heads for cranial deformation analysis. Nevertheless, this closerange photogrammetric experience can be extrapolated to various other scenarios that require the use of low-resolution sensors imaging small and round objects, for instance, industrial applications modelling a series of moving spherical objects, or the documentation of small sculptures in cultural heritage with video recording using smartphones. The number of images was found to be vital to obtain complete models of acceptable accuracy. A high hyper-redundancy photogrammetric approach provided good results in almost every situation. For a low number of images, especially for the second network type with three rings and using the Samsung Galaxy Trend device, insufficient tie points were obtained and image orientation was not possible. Probably an increase in the object's texture would allow completeness of the models with fewer images, as the tie point detection would be easier and more successful. The use of coded targets for the image-orientation step allowed partial models to be obtained in many cases, even when no image orientation was possible without them. For well-defined imaging networks of the three-ring configuration, the targets improved the accuracy significantly. In a particular case, the mean distance changed from $2.9 \mathrm{~mm}$ (unacceptable) to $0.2 \mathrm{~mm}$, which turned out to be the highest accuracy of all models. The calibration of the cameras was found to be the least important aspect of all the parameters evaluated. The calibration methodology (self-calibration with PhotoScan, camera calibration with FOTOGIFLE, and a combination of both) did not improve the final accuracy of the 3D models, even considering both a large number of images and a strong geometric network. One single ring of images was not enough to recover accurately the internal orientation parameters of the camera, even with targets. Therefore, the self-calibration approach can be considered a suitable method for slow-motion smartphone video-image acquisition for close-range photogrammetric applications, whenever configurations of the three-ring type are considered. Different results were obtained for the two devices. The Samsung Galaxy S7, equipped with a more powerful camera, provided better accuracy and much higher reliability than the Samsung Galaxy Trend. However, both devices achieved good results in optimal conditions (high redundancy and coded targets). Therefore, it can be stated that acceptable reliability and accuracy can be obtained using most smartphones in the market, as long as the network conditions are adequate. The network configuration based on the single ring with nearly horizontal images (Fig. 4.4 a) is only recommended when the number of images to be acquired needs to be low and no 
well-defined targets can be used. For these cases, this geometry improved the chance of correct image orientation, but small imperfections in the upper part of the model should be expected. These results can be explained since the images cover mainly the medium-height part of the object, resulting in a high overlap in this area. Because of this fact, the feature detection algorithms performed easier and better, allowing the detection of a higher number of tie points. The hyper-redundancy of the network allows users to achieve complete models even with low-resolution cameras and low-texture objects. However, the larger number of images does not significantly affect the accuracy in the coordinates of targets. Results suggest that the best methodology for this application is the use of a large number of images (95 or more) distributed at different heights around the object. The use of coded targets should be considered, even if it requires extra development to automate the methodology. Under these conditions, it is possible to obtain an error below $0.2 \mathrm{~mm}$, which can be considered totally acceptable as the most common methodologies currently in use have a precision worse than $1 \mathrm{~mm}$ (89).

\subsection{Conclusion}

This paper has assessed the factors affecting the 3D modelling process in order to develop fully automatic and accurate solutions for specific close-range applications. The usage of smartphones for close-range photogrammetry can provide sub-millimetre accuracy whenever the slow-motion video is selected and camera-to-object distances of up to $30 \mathrm{~cm}$ are considered. The number of images required to obtain a fully automatic image orientation is much higher than that needed to obtain high accuracy in classic photogrammetric networks with a small number of manually measured tie points and highly convergent shots. The inclusion of coded targets (whose measurement and matching can be partially automated) has proven to increase significantly the accuracy of the final 3D model. In addition, coded targets facilitate enormously the image orientation, and this is independent of whether a low-end or high-end smartphone camera is used. Three rings of images with coded targets are considered optimal for achieving maximum accuracy with a self-calibration approach, (at least for slow-motion smartphone video image acquisition of a spherical object). Last but not least, highly 
redundant overlapping images are required to achieve correct reliability in the 3D modelling. Further evaluations will be carried out in the future using different cameras, such as stable high-speed cameras, that might yield better calibration results and even better metric deliverables. 


\section{5 \\ Validation of the semiautomatic tool}

This chapter is based on the following paper: Barbero-García, I., Lerma, J. L., Miranda, P., \& Marqués-Mateu, Á. (2019). Smartphone-based photogrammetric 3D modelling assessment by comparison with radiological medical imaging for cranial deformation analysis. Measurement, 131, 372-379. https://doi.org/10.1016/j.measurement.2018.08.059

\subsection{Introduction}

Medical diagnostic imaging techniques, particularly Computed Tomography (CT) and Magnetic Resonance Imaging (MRI) are considered the "gold standard" for the collection of high-quality 3D information in medicine. These techniques have different advantages and limitations. On one hand, they are highly accurate and provide a high level of detail. On the other hand, they are invasive and expensive and CT involves an important dose of radiation. For the particular case of young infants, who would not be still during the test, the techniques are especially invasive since sedation is required. Some authors have studied the validity of models created by photogrammetry and 3D scanners in medicine. Comparison with $\mathrm{CT} / \mathrm{MRI}$ has been carried out for different purposes, including different types of cranial deformation (90, 91), orthodontics (92) and forensics (93), among others. Most studies involve the use of high-cost 3D camera solutions such as STARscanner (Orthomerica, Orlando, FL, USA) or 3D scanners such as 3dMD (3dMD, Atlanta, GA, USA) (26, 71, 90, 91, 92, 94), while others in- 


\section{VALIDATION OF THE SEMIAUTOMATIC TOOL}

clude in-house solutions, such as the combination of several SLR cameras presented by Kottner (93). The most common approach in these studies is the comparison of measurements taken on the $3 \mathrm{D}$ model and those taken directly on the patient. In other studies, the cranial volume is compared (95). Only in some cases, model surfaces are the subject of the comparison (93) but not for cranial deformation analysis. The use of surfaces instead of single measurements provides a more complete comparison between methodologies. Moreover, the whole surface of the model provides a higher amount of information than a limited number of measurements. Different cranial deformation approaches can be used for analysis, such as ellipsoid fitting (61), principal components (26) or global measurement (96). The 3D model also allows the calculation of the cranial volume (95). The use of a smartphone-based low-cost solution for the creation of 3D models for cranial deformation analysis has been introduced by the authors as preliminary tests (61). The image acquisition for this methodology is based on a slow-motion video camera, e.g. coming from a smartphone, and a fitted cap on the patient head. A slow-motion video is recorded in a small time frame during the standard medical consultation while the patient is being held by an accompanying person. Neither special lighting nor tripods are required for the data acquisition. The quality of the models was found to be comparable to that obtained using a full-frame SLR camera for the image acquisition (97). In this study, 3D photogrammetric models are systematically compared with $\mathrm{CT} / \mathrm{MRI}$ models. Image acquisition for the creation of photogrammetric 3D models is carried out using only a smartphone and a fitted special cap in real clinical conditions. Later, the models are compared with either CT or MRI data. The comparison is made, after registration of the different datasets, by computing the minimum distances between the registered photogrammetric and radiological 3D models surfaces. The rest of the chapter is structured as follows. Section 5.2 explains the methodology carried out for the creation of both models (radiological and photogrammetric) and the comparison between them. Section 5.3 presents the results, including distances between models and its significance, as well as the visualization of differences and explanation of error sources. Section 5.4 discusses the results with emphasis on the advantages and disadvantages of the low-cost photogrammetric solution. Finally, Section 5.5 draws some conclusions from the research on deformation analysis. 


\subsection{Methodology}

A total of 10 patients were evaluated (2 females and 8 males). Their age varied between 1 month and 12 years, specifically 4, 5, 9 and 11 months; 1 (two patients), 5, 6, 11 and 12 years. The patients were selected between those undergoing cranial CT or MRI regardless of the existence of cranial deformation to assess the performance of lowcost 3D imaging over CT/MRI. For older patients (over one year of age) long hair was considered an exclusion criterion, as it would affect the creation of the photogrammetric 3D models. The maximum data acquisition time difference between the two data collection approaches was two days; therefore, it can be stated that the data was taken in the same period. For each patient, a smartphone-based photogrammetric model and a radiological model (CT or MRI) were created. Figure 5.1 summarises the main steps carried out for each approach, starting with data acquisition, then processing to build up the 3D models, and finally analysis and comparison after the 3D models are properly registered in the same reference system to determine the distances between them. Further details about the photogrammetric processing to create 3D models from slow-motion smartphone-based photogrammetry have been previously reported (61).

\subsubsection{Radiological data processing}

The cranial CT and MRI scans were performed at La Fe Hospital, Valencia, Spain. The purposes of the test varied for each patient. An important number of patients were under medical control for different types of cranial deformation (preoperative and postoperative stages). The rest of the patients suffered from different pathologies but were always studied with $3 \mathrm{~d} \mathrm{CT}$ or $\mathrm{MR}$ in their follow-up. The data from radiological scans were provided using the Digital Imaging and Communications in Medicine (DICOM) format. The 3D model for each patient was reconstructed using the open-source software InVesalius 3.1.1 (CTI, Brazil). Incomplete models were discarded, although small uncovered areas on the top of the head were allowed. The model was manually segmented for the extraction of the skin and the output was exported to Polygon File Format (PLY). Lastly, the model was manually cleaned and cropped to ease its comparison with the photogrammetric 3D model. 


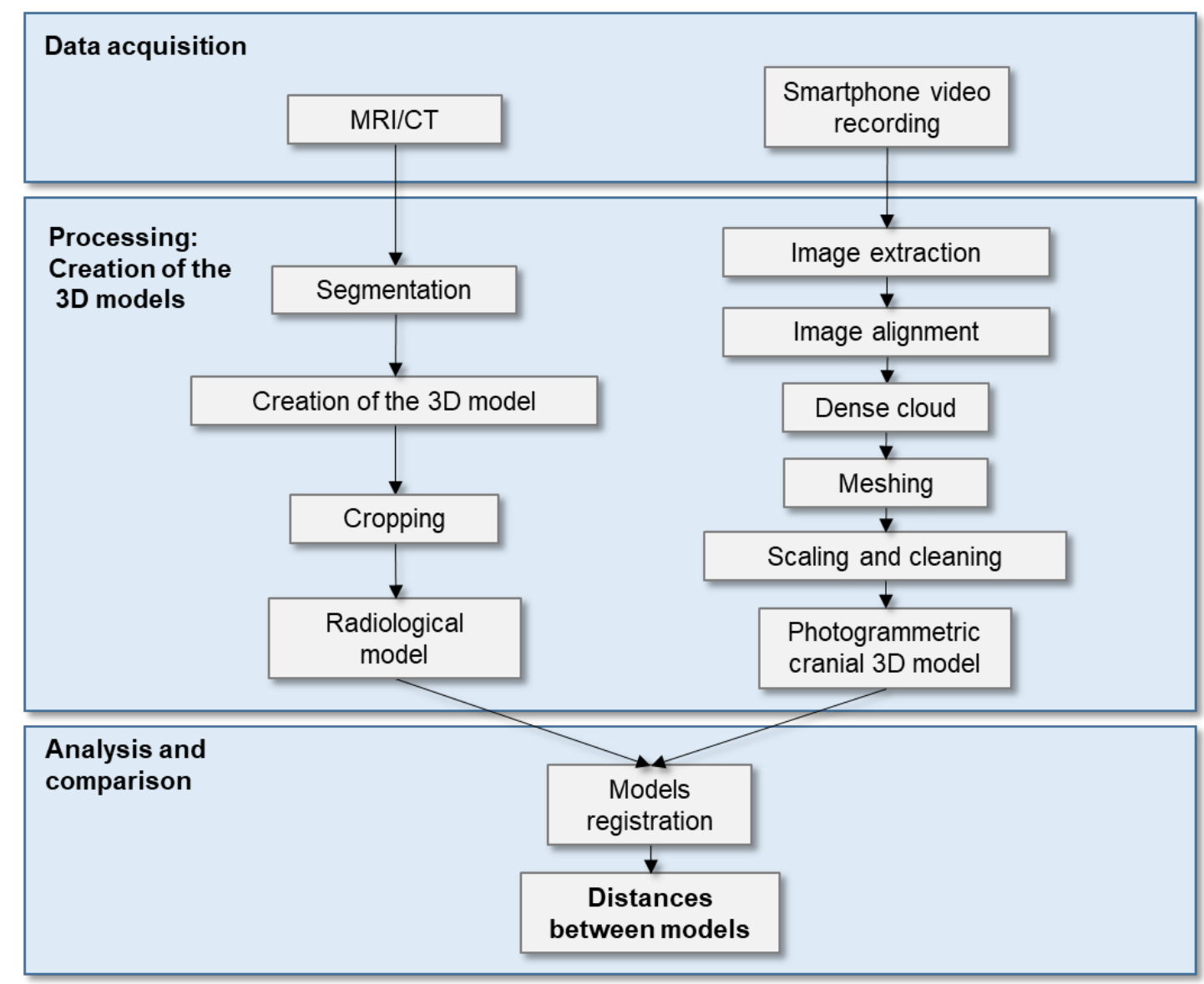

Figure 5.1: Flow diagram used to assess the differences between 3D models. 


\subsubsection{Photogrammetry}

The photogrammetric 3D models were carried out using two different smartphones in slow-motion mode at the maximum frame rate allowed by each device: Samsung Galaxy S7 (Samsung, Seoul, South Korea) with a resolution of 1280x720 at a frame rate of 240 fps and Samsung Galaxy S5 at the same resolution and frame rate of 120 fps. The methodology developed can be found in Barbero-García (61). The video acquisition was carried out during the standard medical consultation. A fitted cap was placed on the patient's head, it was required to avoid the effect of hair in the model and to ease the $3 \mathrm{D}$ reconstruction by adding texture (Fig. 5.2). A detailed study has been carried out to determine the smartphone performance for photogrammetric smartphone-based applications on spherical objects (98). After testing two smartphones, one high-end Samsung Galaxy S7 (2017) and another Samsung Galaxy Trend Plus (2014), no special specifications were actually found as limiting factors other than high definition (HD, $1280 \times 720$ pixels) video and the slow-motion acquisition. In any case, a recording taken in three rings with large overlap was found essential for automatic full 3D modelling without imperfections. Image acquisition The illumination conditions in the room were kept at a maximum to compensate for the lack of exposure of the slow-motion video mode and assure the correct focusing of the mobile device. However, no special lighting was required. In case of early age patients, they were held upright by an adult. The patients were not asked to stay still, so normal movements occurred, especially in the case of younger infants. A video was recorded for each patient, lasting a maximum of 30 s. During the video recording, the operator moved around the patient's head in three rings with the smartphone at different heights (viewpoints). This approach is based on the conclusions of previous studies carried out by the authors. Indeed, the optimal geometry of the image acquisition approach for near-spherical objects (e.g. heads), using conventional, non-metric video cameras integrated into current smartphones can be found in Barbero-García et al. (98). The whole image acquisition process required less than five minutes per patient and was carried out during a standard consultation. Special care must be taken to conveniently cover the whole head surface in the video sequence, which can be hampered by the constant quick movements of the infants. It is worth noticing that this methodology requires the cap to be correctly fitted on the 
head to avoid imperfections during the eventual processing for 3D reconstruction and dense image matching.

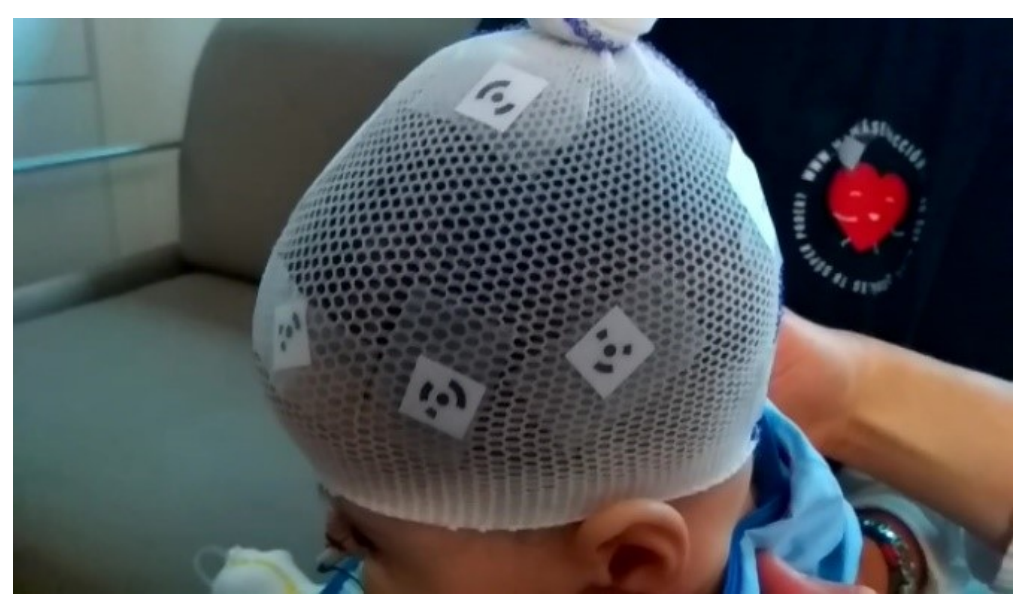

Figure 5.2: Image used for the creation of the 3D model.

\subsubsection{Processing}

Firstly, the images were manually extracted from the video. Between 200 and 300 images were selected in each video file. The creation of the $3 \mathrm{D}$ models was carried out using PhotoScan (Agisoft, Russia). The 3D modelling process followed the usual pipeline in photogrammetric Structure-from-Motion (SfM) software, consisting of (i) Image alignment, (ii) Cloud densification, (iv) Meshing and (v) Texturing the mesh (optional, for visualisation purposes only). In case of failure, the process was completed using manually defined tie points. The model was then scaled using targets of known dimensions placed on the cap. Finally, areas outside the cap were cropped to avoid any measurements in those areas. The final 3D models obtained for each patient had between 100.000-200.000 points and 200.000-300.000 faces. The expected accuracy of the models was $1 \mathrm{~mm}$ (97). A camera self-calibration was automatically done during the spatial orientation processing in the photogrammetric software as no previous camera calibration was available for each one of the sessions. Figure 5.3 displays an image with the footprint of the geometric distortion pattern of the smartphone camera in one of the sessions. The self-calibration approach includes a comprehensive calibration to determine its interior orientation parameters, i.e. the principal distance, principal point offsets, radial/decentring lens distortion parameters and the affinity parameters 
(differential scaling and skewness). A calibration of the camera prior to the image acquisition was discarded as it would require a high amount of time and knowledge, making the methodology difficult to be applied in the clinical practice. Moreover, previous studies show that prior calibration of the camera does not have an important impact on the results for this particular application as far a large number of images are selected for the camera calibration (98).

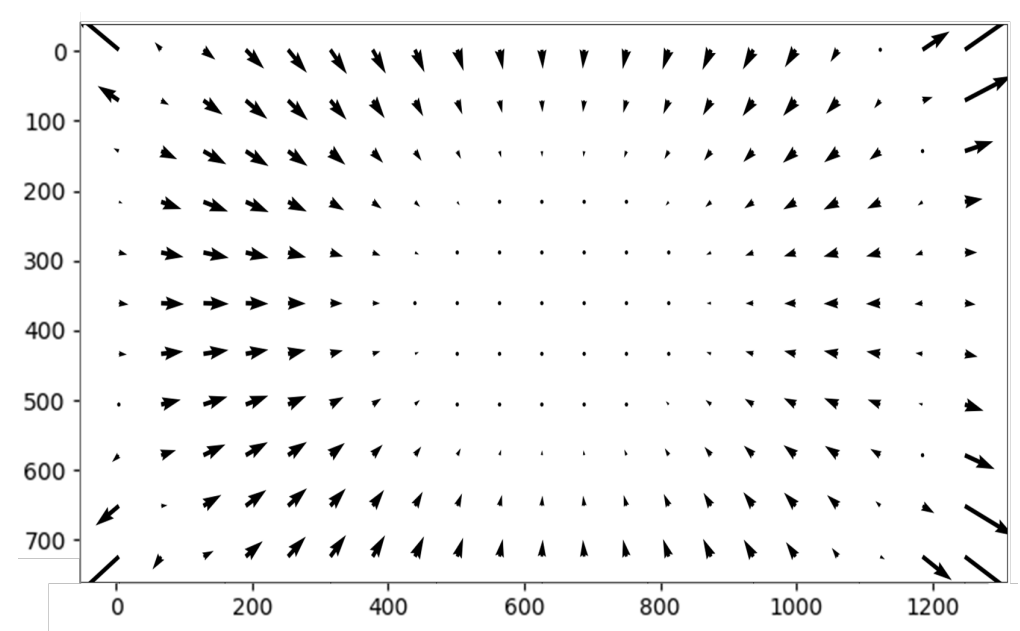

Figure 5.3: Calibration distortion pattern for Samsung Galaxy S5 (Scale: 2x).

\subsubsection{Comparison}

The software CloudCompare version 2.7.0 (http://cloudcompare.org/) was used to compare each pair of photogrammetric and radiological 3D models. In order to allow the comparison, the 3D models were manually registered to the same coordinate system. The registration was carried out by manual identification of cranial landmarks and posterior minimisation of the distance between models. The distance between models was computed for every point in the photogrammetric model (between 70.000 and 100.000 points) as the distance to the closest point of the radiological model. The tool "Compute cloud/mesh distance" in CloudCompare was used for this calculation, the option "signed distances" was chosen, as it is important for the evaluation; the radiological model was always used as a reference. A small shift between two models $(2 \mathrm{~mm}$ approx.) was expected due to the effect of the cap on top of the head pressing the hair, as pointed out by different authors (무, 90). 


\section{VALIDATION OF THE SEMIAUTOMATIC TOOL}

\subsection{Results}

The differences between models can be found in Table 5.1 which contains the 10 patients ordered by age. The control radiological technique used for the comparison is specified in the fourth column with header CT/RMI. The maximum time shift between radiological imaging and photogrammetric data acquisition was less than 2 days. The average mean difference distance between pairs of models (Photogrammetry - CT/MRI) is $2.1 \mathrm{~mm}$, being the minimum $0.5 \mathrm{~mm}$ and the maximum $3.2 \mathrm{~mm}$. The standard deviation of the distance differences is constant across patients (0.7-1.4) with an average of $1.2 \mathrm{~mm}$. Table 5.1. Patients' information and distance difference parameters between radiological and photogrammetric models (differences in $\mathrm{mm}$ ).

\begin{tabular}{ccccccc}
\hline $\begin{array}{c}\text { Patient } \\
\text { number }\end{array}$ & Gender & $\begin{array}{c}\text { Age } \\
\text { (months) }\end{array}$ & CT/MRI & $\begin{array}{c}\text { Time dif. } \\
\text { (days) }\end{array}$ & $\begin{array}{c}\text { Mean } \\
\text { distance }\end{array}$ & $\begin{array}{c}\text { Distance } \\
\text { standard } \\
\text { deviation }\end{array}$ \\
\hline 1 & $\mathrm{M}$ & 4.3 & $\mathrm{MRI}$ & 0.0 & 1.9 & 1.1 \\
2 & $\mathrm{~F}$ & 5.8 & $\mathrm{MRI}$ & 2.0 & 0.5 & 0.7 \\
3 & $\mathrm{M}$ & 9.5 & $\mathrm{CT}$ & 0.0 & 1.5 & 0.7 \\
4 & $\mathrm{~F}$ & 11.1 & $\mathrm{CT}$ & 1.0 & 1.3 & 1.3 \\
5 & $\mathrm{M}$ & 17.5 & $\mathrm{MRI}$ & 0.0 & 2.0 & 1.2 \\
6 & $\mathrm{M}$ & 21.8 & $\mathrm{CT}$ & 0.0 & 2.4 & 1.4 \\
7 & $\mathrm{M}$ & 70.4 & $\mathrm{CT}$ & 0.0 & 2.4 & 1.1 \\
8 & $\mathrm{M}$ & 81.2 & $\mathrm{MRI}$ & 1.0 & 3.2 & 1.3 \\
9 & $\mathrm{M}$ & 142.6 & $\mathrm{MRI}$ & 1.0 & 2.6 & 1.4 \\
10 & $\mathrm{M}$ & 147.1 & $\mathrm{CT}$ & 0.0 & 1.1 & 1.4 \\
\hline & & & & Average & 2.1 & 1.2 \\
\hline
\end{tabular}

Table 5.1: Patients' information and distance difference parameters between radiological and photogrammetric models (differences in $\mathrm{mm}$ ).

The Student t-test was carried out for both the mean difference distance and the standard deviation. The $99 \%$ confidence interval for the mean difference distance is 1.1$2.7 \mathrm{~mm}$. As for the standard deviation, the test confirms that the value is below $1.4 \mathrm{~mm}$ with a $99 \%$ confidence. The mean difference distances and their standard deviations (Y axis) in relation to age (X axis) are presented in Figure 5.4 . For patients younger than two years $(\mathrm{n}=6)$, the maximum difference distance is $2.4 \mathrm{~mm}$ and its average difference distance is $1.6 \mathrm{~mm}$; this is the normal age range for cranial deformation assessment. Lower differences for this age range are possible, as hair does not affect the models. 


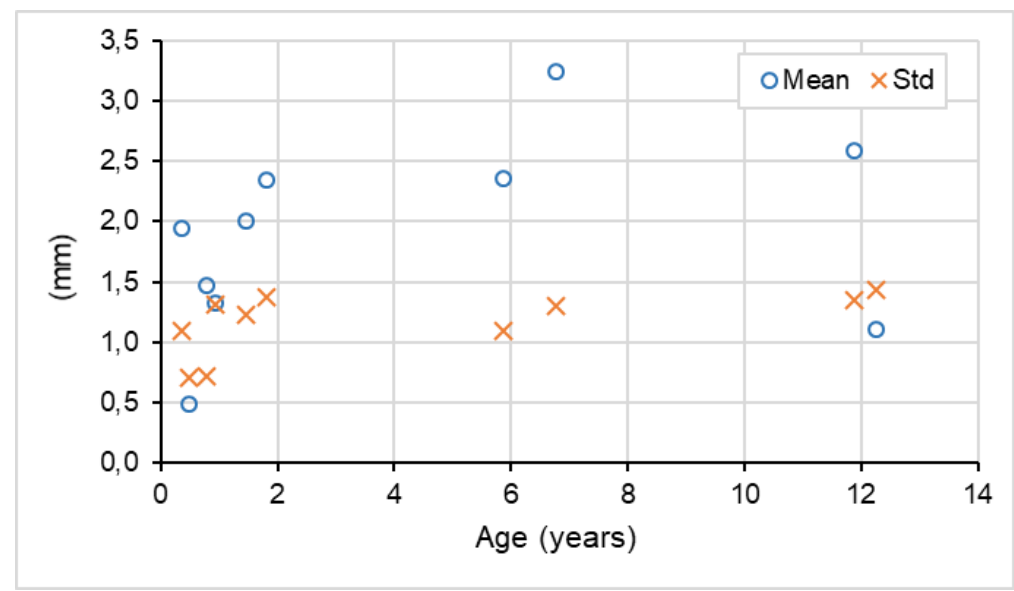

Figure 5.4: Mean distance differences and their standard deviations in relation to their patients' age.

Figure 5.5 shows the mean distance and standard deviation for both age groups: up to two years of age (Fig. 5.5p) and above two years (Fig. 5.5c). Mean distance is considerably higher for the older patients group (Under two years: $1.6 \mathrm{~mm}$, above two years: $2.3 \mathrm{~mm}$ ). The comparison values are also shown for CT and MRI techniques separately, the mean difference is higher for MRI (MRI: $2.05 \mathrm{~mm}, \mathrm{CT}: 1.72 \mathrm{~mm}$ ) while the standard deviation is similar (MRI: $1.1 \mathrm{~mm}, \mathrm{CT}: 1.2 \mathrm{~mm}$ ).

Three-dimensional models of each patient's head were delivered (Figs. 5.6 and 5.7). Figure 5.6a, b shows the frontal and lateral views of one patient after CT, whereas Figure 5.6,, c exhibits views after MRI. Colour maps were obtained for each patient showing the difference distances between 3D models delivered by CT and photogrammetry (Fig. 5.6, d) and MRI and photogrammetry (Fig. 5.7p,d). Overall, no systematic errors were found as can be checked in Figure 5.6. Areas of higher difference are located randomly around the head and particularly in the edge of the model.

The most important sources that yield a difference between radiological and photogrammetric 3D models were caused by the combined effect of the hair and the cap; and, in some cases, small inaccuracies of the photogrammetric model caused by low texture areas and edges. However, small errors were also found in some radiological 3D models (Fig. 5.7a,b). Another source of error was given by the lying position of the patients that, in some cases, resulted in skin folds that were also detected as distance differences between models (Fig. 5.7c,d). 


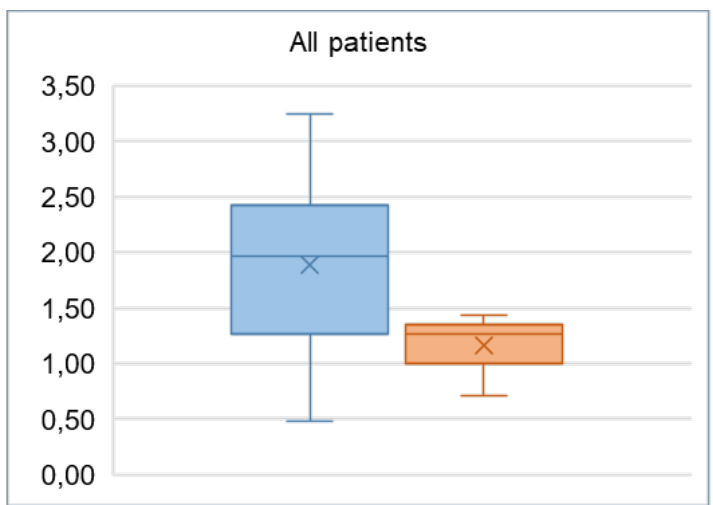

$\square$ Mean distance

Distance standard

(a)

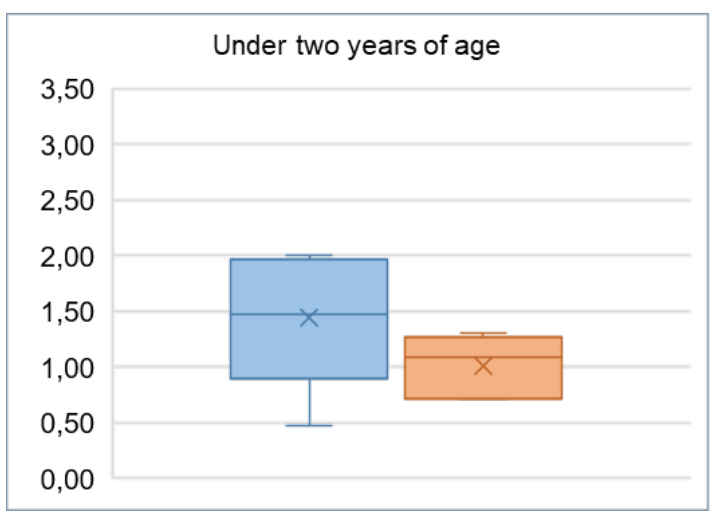

(b)

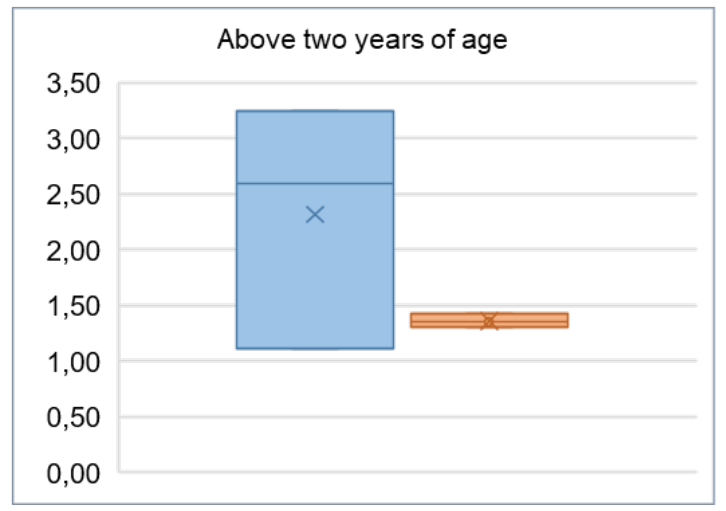

(c)

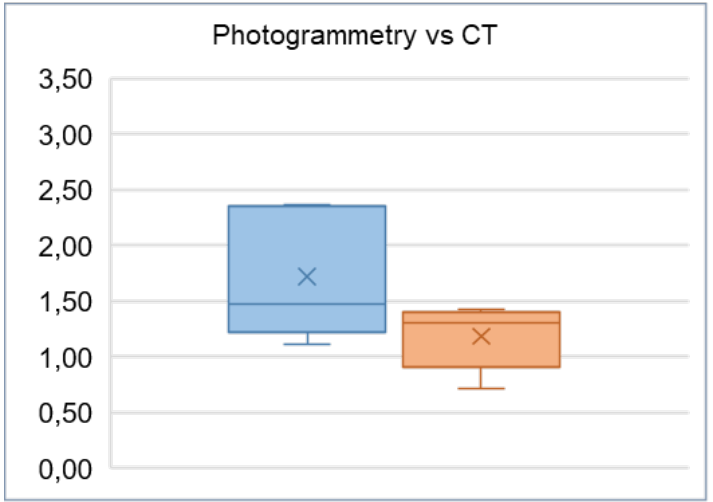

(d)

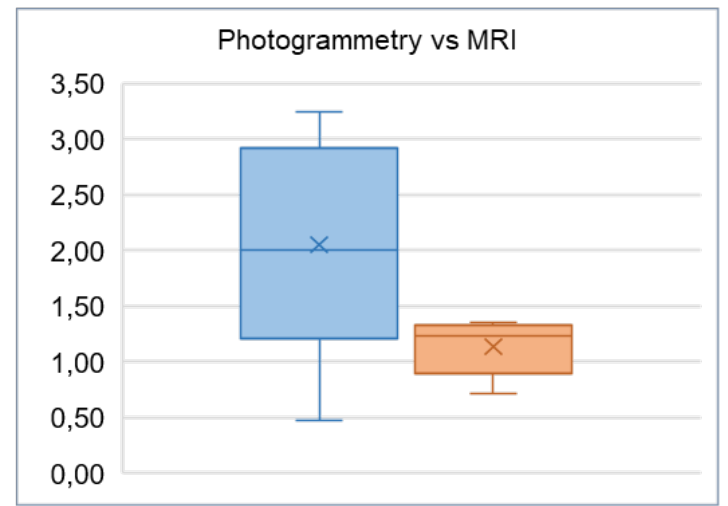

(e)

Figure 5.5: Photogrammetry vs radiological test box-and-whisker plots showing the mean distance and the standard deviation results: a) all patients, b) under two years of age, c) older than two years, d) CT, and e) MRI. 


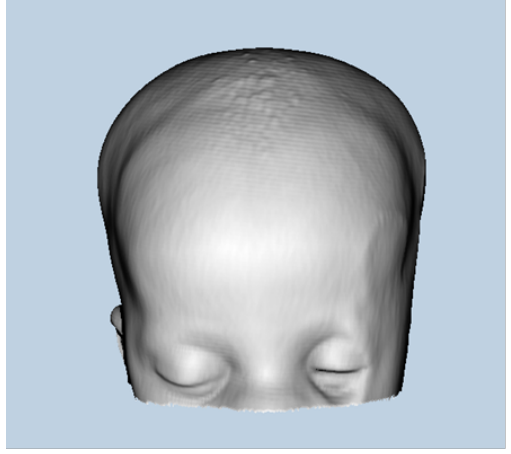

(a)

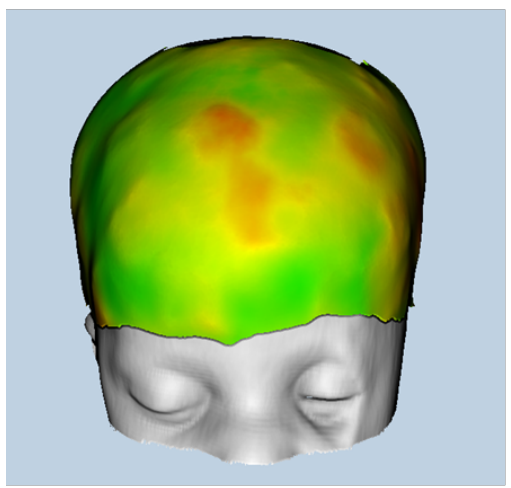

(c)

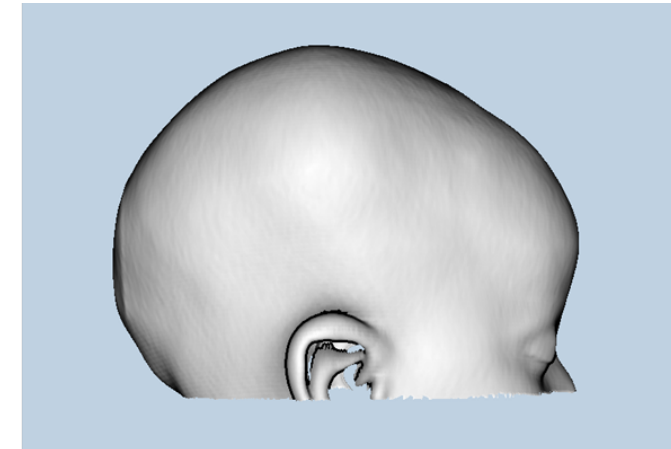

(b)

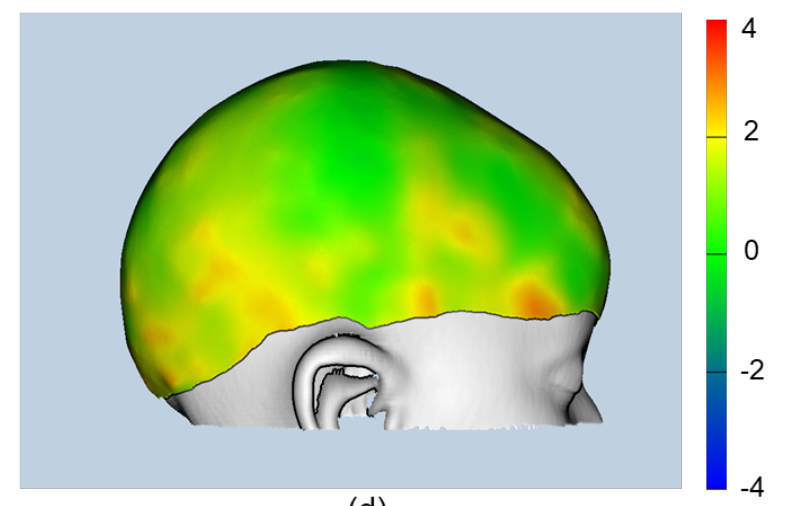

(d)
Distance $(\mathrm{mm})$

Figure 5.6: Derived models for the 3rd patient: a, b) CT; c, d) Difference distances in the 3D model between the photogrammetric solution and the registered CT. 


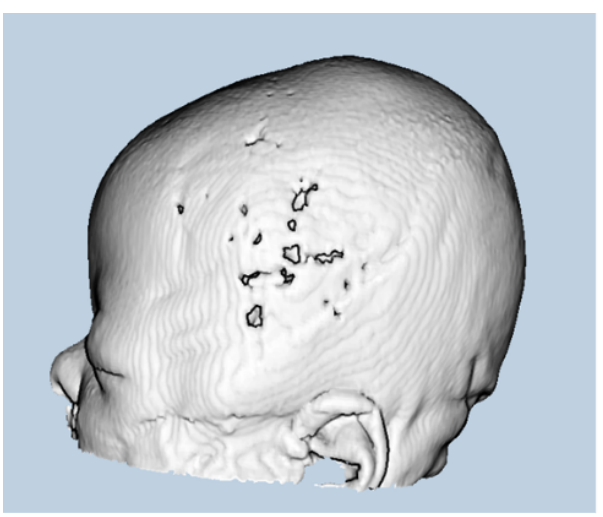

(a)

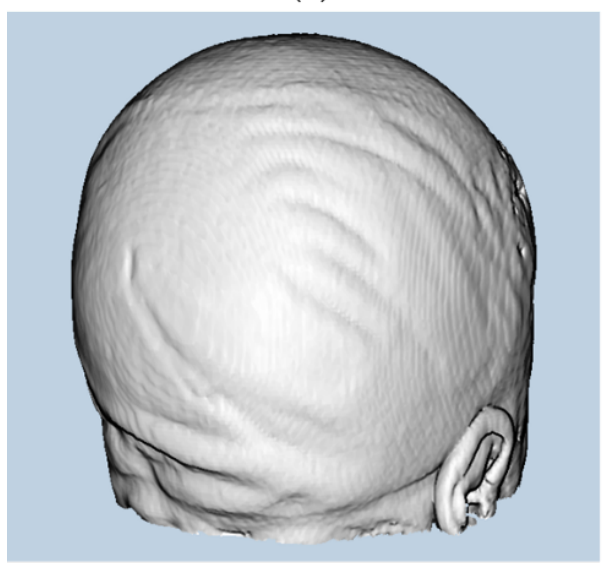

(c)

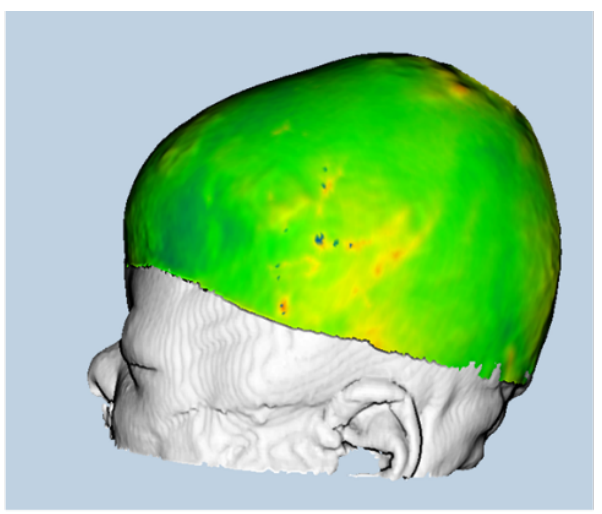

(b)

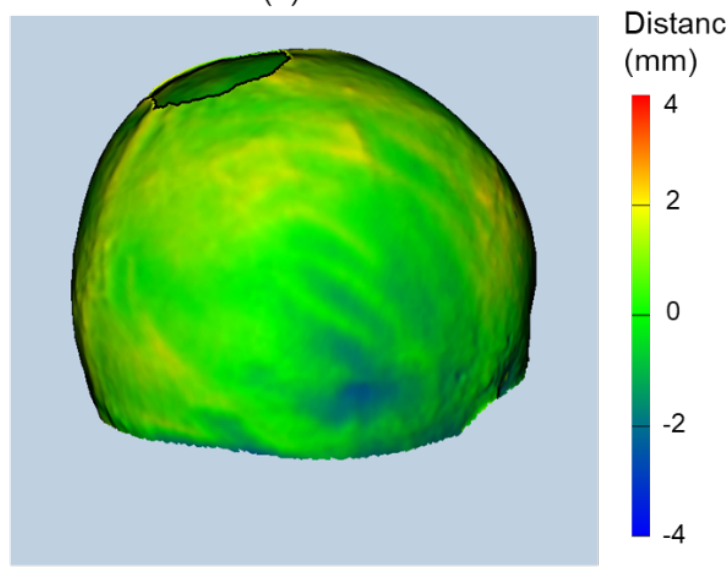

(d)

Figure 5.7: Examples of errors found in MRI 3D models due to holes (a) and lying position of the patient during the data acquisition (c). These errors affect the distance differences between photogrammetry and MRI (b, d), respectively. 


\subsection{Discussion}

The evaluation and monitoring of cranial deformation is usual practice during paediatric consultations. However, the commonly used techniques, including visual assessment and the use of callipers and measuring tape, are strongly limited and experts do not agree on their reliability and capacity to represent adequately the deformation (20, 96). Specifically designed 3D scanners and setups of 3D cameras have become an alternative for the evaluation of cranial deformation (such as STARscanner and 3dMD) $(93,94,96)$. They are especially useful for the evaluation of cranial deformation as they provide highly accurate and complete information on the patient's head shape and they are non-invasive. However, due to the high cost of the metric devices, the methodology is not implemented as part of the regular clinical practice. The methodology assessed in this study based on smartphone photogrammetry provides similar results to 3D scanners and multi-camera approaches. Moreover, it is low cost as only a smartphone is required for data acquisition. The simplicity of the setup would allow a real implementation of the methodology in routine clinical practice once a full automatic toolbox is developed. Although some studies have evaluated the usability of 3D models for cranial deformation evaluation, the authors have not found references in the literature comparing the radiological and photogrammetric 3D models applying the surface distance differences method. This technique provides a significantly higher amount of information than the comparison of a limited number of manual measurements. The results showed an overestimation of $0.48-3.24 \mathrm{~mm}$, although other authors reported that an overestimation of approximately $2 \mathrm{~mm}$ was to be expected (90). However, distance differences in shape are low and constant, with standard deviations below $1.5 \mathrm{~mm}$ for all patients. Most distance differences in the 3D models are local and due to low texture areas, near the edges of the model or imperfections of the MRI/CT. Therefore, the imperfections in the 3D modelling will not be important enough to affect the assessment of the cranial deformation by the doctors and medical specialists. It should be taken into account that higher distance differences happened only in older patients. The maximum mean distance difference for two-year-old infants is $2.35 \mathrm{~mm}$. The reason for the older patients overestimation is surely the hair effect. Although patients with long hair were excluded from this study it was noticed that even thick, short hair can affect the results. This was not a problem with younger infants. Thus, hair has to 


\section{VALIDATION OF THE SEMIAUTOMATIC TOOL}

be taken into account as a limiting factor for the effective application of this low-cost photogrammetric technology. However, infants subject to cranial deformation analysis in different stages, both pre and post-surgical, are usually under one year old. In fact, most cranial deformations appear during the first months of life (99). In addition, the ideal age for starting a correction is below 6 months according to some authors (9), while others suggest that correction should start before four months (100). Besides, the use of helmets is considered especially useful in the age range of 4 to 12 months. Therefore, the presented methodology is expected to cover the right age frame when both diagnosis and monitoring are found essential. Nevertheless, the only known constraint for applying this low-cost methodology is the presence of short hair, but it is not usually a problem in infants up to 1-year-old. The usage of a perfectly fitted cap is totally necessary to obtain accurate results. As a consequence of this, any patient with head bandages that could not be removed or any other unremovable devices, such as cranial distractors, would need adequate customization of the reference cap or otherwise would not be eligible for this low cost smartphone-based photogrammetric methodology. The movement of patients during the data acquisition is also an issue for younger infants, however, no inaccuracies were found as a consequence the of movement. The main limitation of the methodology is the impossibility to extract non-visible information such as those on the bone. However, most common cranial deformation types, such as positional plagiocephaly, are measured using the surface information only. For severe cases of deformation, such as craniosynostosis, this methodology could not be used as a diagnostic tool but it could be combined with radiological tests for monitoring purposes, especially for patients following a treatment such as cranial orthosis. Currently, the main tools for the measurement of cranial deformation are the metric tape and the calliper. These tools are intended to acquire isolated craniometric indexes. Although clinically useful, these indexes do not provide a 3D representation of the addressed deformity. Besides, in optimal conditions, the precision of this methodology is $1 \mathrm{~mm}$. However, in real clinical conditions (including infants moving, hampering the identification of cranial landmarks) accuracy is worse than $2 \mathrm{~mm}$ and shows a significant interobserver and intraobserver variability. The human tolerance for the perception of mild head asymmetry is also considered $2 \mathrm{~mm}$ (101). As the presented results show differences in shape compared to radiological tests below $1.5 \mathrm{~mm}$ we consider that the methodology can provide at least similar accuracy to traditional clinical measurements, 
but with the advantage of having a complete 3D objective model as output and in a totally non-invasive and low-cost manner. The implementation of the methodology could mean an easier and more detailed evaluation of infants from early ages, allowing better monitoring of the patient's evolution and the results of the treatments.

\subsection{Conclusion}

Photogrammetric 3D models obtained from smartphone-based slow-motion videos have been found to provide valuable information for the assessment of cranial deformation in infants under 2 years of age. The differences with the "gold standard" represented by CT and MRI show an overestimation of the photogrammetric caused, by the effect of the hair and the cap. The average difference distance in shape determined from the 10 full 3D models of the patients' heads was $2.1 \mathrm{~mm}$; and $1.6 \mathrm{~mm}$ for infants under 2 years of age. The standard deviation of differences is below $1.5 \mathrm{~mm}$ for all patients. These values clearly validate the proposed smartphone-based photogrammetric solution as it has similar accuracy to other commonly used methodologies such as callipers or measure tape, but with higher reliability and repeatability and in a comprehensive way. i.e. covering the whole patient's head. The main disadvantage of the presented methodology is its limitation to extracting outer visible information only. As a consequence, it can replace radiological tests as far outer anomalies are presented in infant's patients. Nevertheless, the value of the presented methodology is bounded by the possibility to include it in the regular clinical practice as a routine monitoring, non-invasive technique. Therefore, future research should focus on the development of a fully automatic tool able to deliver ready-to-use 3D models and reports to doctors and medical staff. The availability of cranial 3D models would allow the development of new deformation assessment parameters adapted to detailed and comprehensive 3D data. The possibilities in this area have been partially explored and more development is foreseen in the near future. 


\section{6}

\section{Registration of the models}

This chapter is based on the following paper:

Barbero-García, I., \& Lerma, J. L. (2019). Assessment of Registration Methods for Cranial 3D Modelling. Proceedings, 19(8). https://doi.org/10.3390/proceedings2019019008

\subsection{Introduction}

Nowadays, the use of 3D models for cranial deformation assessment is becoming more common, but the methodology still needs to be improved (43). The authors have presented a low-cost, smartphone-based methodology for head 3D model creation (61) and its accuracy has been evaluated by comparison with radiological imaging (102). The registration of the head 3D models coming from different devices and arbitrary 3D technologies to a common reference system is still a challenge. For the purpose of monitoring cranial deformation in infants, model registration is required in order to calculate deformation indexes and compare multitemporal models. Most 3D model solutions (e.g., STARScanner, Orthomerica, Orlando, Florida, USA; 3DMD, Atlanta, Georgia, USA) cover the whole area of the head, including the face, and use craniometric and facial points to register the model (43). These methodologies are costly so their use is not widespread as part of the clinical routine. In cases where bone information is acquired (radiological images), bone structures are used to identify craniometric points. For the developed application, the area in the 3D model is given by the area covered by a fitted cap, so the face is not registered. The identification of landmarks on the model is almost impossible, hampering the registration. The proposed solution includes 
the manual identification of tie points by the medical staff. The identified tie points are later used for automatic registration in combination with PCA. Two methodologies are tested, using two and three manually identified tie points. The results are also compared with registration using PCA only.

\subsection{Methodology}

\subsubsection{Data acquisition}

The head 3D model data acquisition consists of 3 steps: (i) Placing a fitted cap on the infant's head, (ii) Locating the required craniometric points using stickers and (iii) Recording a slow-motion video of the infant head using a smartphone. The position of the cap can vary significantly every time it is placed on an infant head (it can be placed to cover a bigger part of the forehead and/or nape, or go lower at one side). The required craniometric points can also have small differences in location. To evaluate the effect of cap and tie point position changes on the registration, the cap and points were put on a static model a total of 6 times. For each position, two data acquisitions were carried out, resulting in 12 models. The models were created using a smartphone camera and Agisoft PhotoScan as described by the authors in a previous paper (61).

\subsubsection{Registration}

The registration process was carried out for each model using three different methodologies: (i) PCA without tie points; (ii) PCA plus two tie points (glabella and opisthocranion); and (iii) PCA plus three tie points (glabella and both pre-auricular points).

\subsubsection{PCA plus two tie points}

The identified tie points were glabella and opisthocranion. After PCA, the model was translated, so the origin of the coordinate systems was the mid-point between both tie points. The model was later rotated to match glabella-opisthocranion line with the $\mathrm{x}$-axis.

\subsubsection{PCA plus three tie points}

The identified tie points were glabella and both pre-auricular points. After PCA the model is rotated and translated so the plane given by the 3 tie points matches $\mathrm{z}=0$, 


\section{REGISTRATION OF THE MODELS}

the line given by preauricular points matches y-axis and the line given by glabella and the line given by pre-auricular points matches x-axis (Fig. 6.1a,b).

\subsubsection{Distance calculation}

The differences were calculated as distances between meshes (Fig. 6.1). For each pair of meshes, a reference one was chosen. For each point in the reference mesh, the distance to the second mesh was computed along the direction defined by the reference point and the coordinate system origin. This methodology improves the Iterative Closest Point (ICP) algorithm and fits the normal direction avoiding texture influence. It also has a lower computational cost than any ICP approach (103).

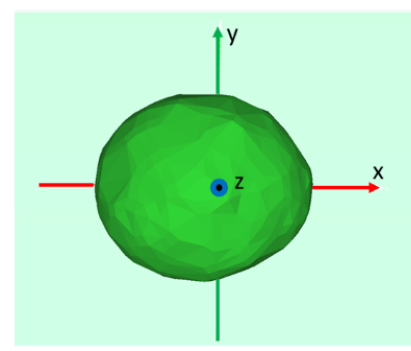

(a)

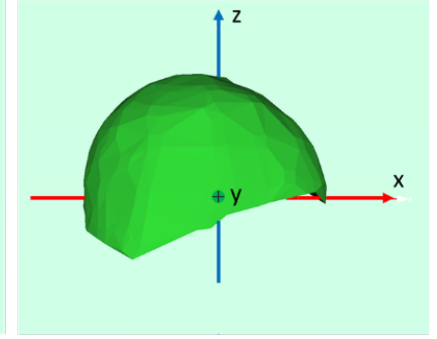

(b)

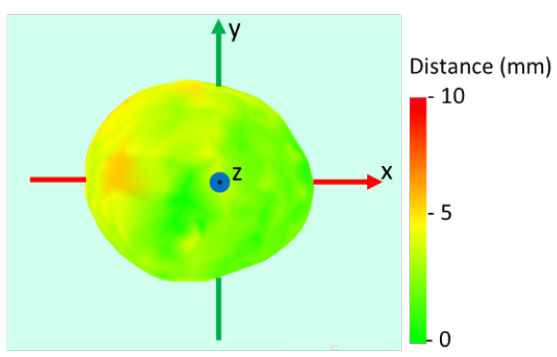

(c)

Figure 6.1: Top (a) and lateral (b) view of a model registered using CPA plus three tie points. Distances between two models (c).

\subsection{Results}

All the possible combinations between models were made for each methodology. The results are compared in general and for the same cap positions only (Table 6.1).

\begin{tabular}{ccccccc}
\hline & \multicolumn{2}{c}{ PCA without Tie Points } & \multicolumn{2}{c}{ PCA Plus 2 Tie Points } & \multicolumn{2}{c}{ PCA Plus 3 Tie Points } \\
\hline $\begin{array}{c}\text { Average } \\
\text { distance }\end{array}$ & Mean & Std & Mean & Std & Mean & Std \\
\hline $\begin{array}{c}\text { All } \\
\text { Same }\end{array}$ & 4.6 & 3.1 & 3.4 & 2.0 & 1.9 & 1.1 \\
position & 0.7 & 0.6 & 1.6 & 0.9 & 1.5 & 0.7 \\
\hline
\end{tabular}

Table 6.1: Distances between registered models ( $\mathrm{mm})$. 


\subsection{Discussion}

PCA without tie points showed high errors for the whole set of 3D models but the results without varying the cap positioning are much better. As it was to be expected, the methodology is greatly affected by the position of the cap. PCA plus two tie points moderately improved the results of the first method, although the results achieved under the same position increased the distances. The distance increment is explained partly by a model that presented high error for one of the identified points, causing the whole model to be badly registered. PCA plus three tie points yielded the best results for the whole set of models, with distances below $2 \mathrm{~mm}$. However, the distances for pairs of models taken without moving the cap were higher than those obtained using the PCA only.

\subsection{Discussion}

Accurate registration of the head 3D models is vital for obtaining useful information for cranial deformation assessment. This requirement becomes harder to achieve when the model does not include easily-identifiable points such as craniometric facial points or cranial information. PCA was considered an interesting option as it easily identifies the maximum variance axis. However, it was pointed out that it is very dependent on the position of the cap and different examiners usually place the cap differently. To overcome this limitation a methodology has been developed based on the combination of PCA and tie points manually identified by the medical staff. The number of points must be limited so that it does not increase the data acquisition time. Furthermore, the required points must be easily identifiable in a moving infant. Firstly, an approach based on only two tie points was tested. Opisthocranion and glabella were chosen, as they are important points for cranial deformation indexes. However, a problem found is the difficulty to identify opisthocranion reliably. A second approach replaced opisthocranion by the two pre-auricular points, left and right, which are easier to identify. Increasing the number of points to three allows defining a plane and therefore registering the model without the need for more data. The results show that registration using PCA plus three tie points is independent of the cap position, so it is more applicable in real life. The main disadvantage of the point-based registration is that the manually identified tie points are more subject to errors, as the registration is based on a very reduced number of points. This problem is worsened, as the points are located 


\section{REGISTRATION OF THE MODELS}

on the edge of the model, where more distortions are likely to happen. Nevertheless, promising results are expected after changing slightly the registration methodology.

\subsection{Conclusions}

A registration approach using PCA has been found to have enough accuracy for analysing cranial deformation. The method is useful as users are only required to identify three tie points (which can be stuck to the cap) and the registration can be fully automated. In the future, we are planning to slightly improve the presented registration methodology. Eventually, the methodology will be tested under real clinical conditions 


\section{7 \\ The automatic tool}

This chapter is based on the following paper:

Barbero-García, I., Lerma, J.L., Mora-Navarro, G., 2020. Fully automatic smartphonebased photogrammetric 3D modelling of infant's heads for cranial deformation analysis.

ISPRS J. Photogramm. Remote Sens. 166, 268-277. https://doi.org/10.1016/j.isprsjprs.2020.06.013

\subsection{Introduction}

In this chapter, the novel and fully automatic solution for the creation of the infant's head 3D models is presented. The solution consists of a mobile app, a coded cap and processing software. First, the cap is placed in the infant's head. Then a doctor or any user would use the mobile app to get the necessary data for the creation of the $3 \mathrm{D}$ models. The whole process is guided so the user is not required to have any knowledge of photogrammetry. Once all the required data is collected, it is sent to a server where it is processed to obtain the final $3 \mathrm{D}$ model, which is presented to the doctor in several minutes.

For the accurate evaluation of cranial deformation, an accurate 3D model of the patient's head (infants ranging usually from 1-24 months old) must be delivered during a normal consultation, in a way that the medical staff can make right diagnosis and decisions. In order to create a useful and low-cost medical device, it is vital to deal with the movement and produce highly accurate 3D models in real clinical conditions. The use of photogrammetric devices by non-experts, such as medical staff, requires a high degree of automation and reliability (104). It is also important to extract the 


\section{THE AUTOMATIC TOOL}

information in short periods as the consultation time is, in most cases, very limited. Besides, the metric solutions have to be robust and reliable enough to satisfy challenging health demands.

The automation possibilities are different regarding the requirements and conditions of every particular medical application. The use of coded markers, under different setup geometries and the required number of images to extract useful infant's head 3D models have been explored by the authors (98). The accuracy of manually-driven 3D models has also been tested by the authors in comparison with the present gold standards, CT and MRI (102). However, a fully automatic methodology for the extraction of the 3D models has not been presented.

Automatic camera pose estimation and calibration are usually carried out using automatically detected keypoints. The obtainment of these keypoints is commonly carried out using algorithms such as SIFT (76) or SURF (77), or more novel robust approaches based on ASIFT (105). Obtaining keypoints is time-consuming when dealing with large datasets. For moving objects, some type of masking is required to assure that the keypoints are located in the object and not in the background. The target-based approach is an alternative to the keypoints approach for camera pose estimation. It is especially useful for low-texture areas where not enough keypoints can be identified (106). The target-based approach is not always an option as the placement of the targets is not possible in many situations when the object to be modelled cannot be altered. For this particular application, a cap is placed on the patient's head and the texture of the model does not add any useful information. For this reason, the placement of markers is not an issue. Moreover, with this approach, the movement of the background will not be a problem, as all markers are placed on the cap. The targets chosen for this study are ArUco markers. ArUco markers include a black frame that allows quick detection and binary code for identification. For every marker, four points (corners) can be automatically identified with subpixel accuracy (107, 108). This type of markers has been used for the calibration of low-cost cameras (109). 


\subsection{Materials and methods}

\subsubsection{Automatic PhotoMeDAS tool}

The PhotoMeDAS (Photogrammetric Medical Deformation Assessment Solutions) patentpending tool is composed of coded cap, coded stickers, mobile app and processing software. The mobile application is used together with the cap and stickers to carry out the data acquisition. The software, located in a server, processes the data and creates a 3D model. It also obtains some head shape information and deformation parameters, which are available to the user through a webpage service (Fig. 7.1).

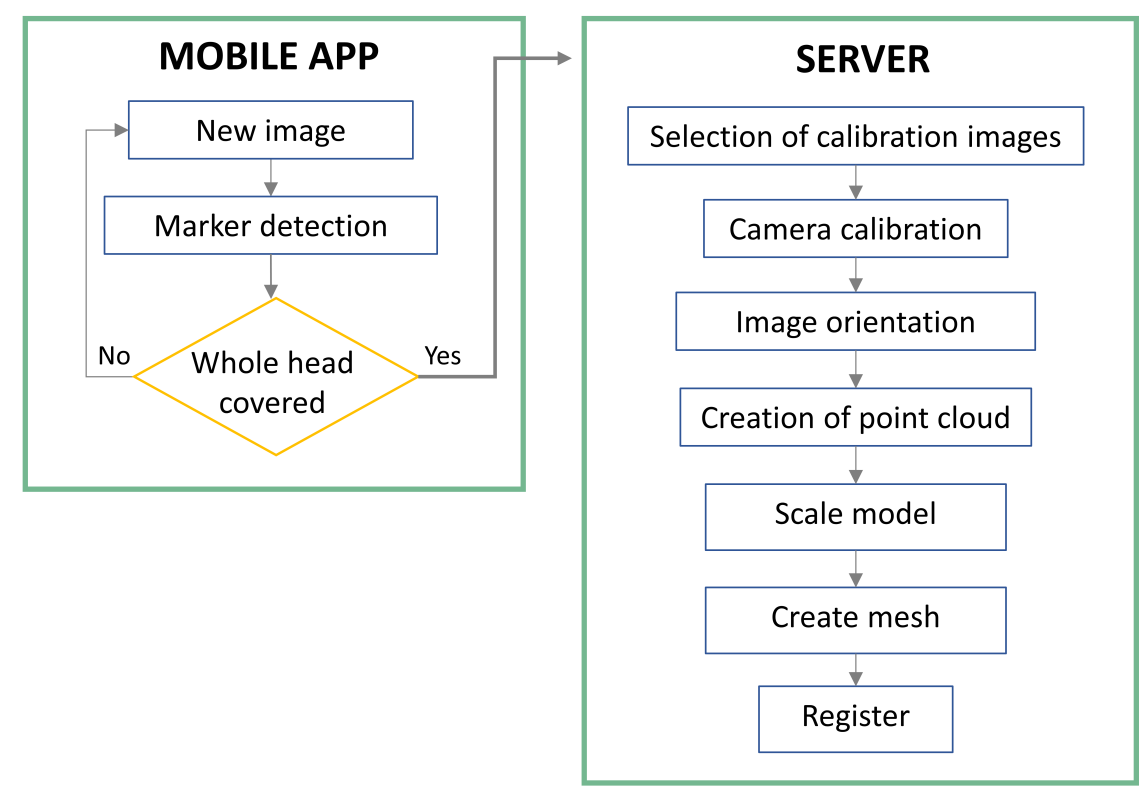

Figure 7.1: Implemented photogrammetric workflow.

\subsubsection{Coded cap}

The cap is made of an elastic material on which coded ArUco markers have been added. Each cap contains a total of 131 markers, the material of the markers is not elastic to assure their size is constant and can be used to scale the model (Fig. 7.2). Together with the cap, three stickers are provided. The stickers are similar to the markers present on the cap. The medical staff carrying out the image acquisition are asked to place one of them between the eyes and the others on the left-hand side and right-hand side 


\section{THE AUTOMATIC TOOL}

pre-auricular points. The identification of these points will be used for the registration of the models (monitoring) over time (Fig. 7.2).

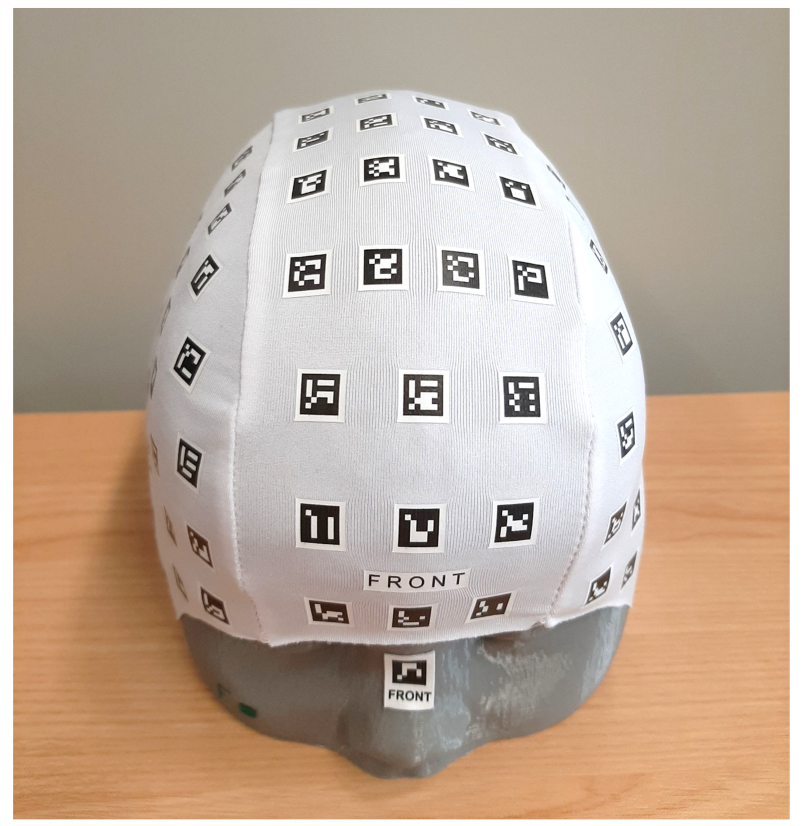

(a)

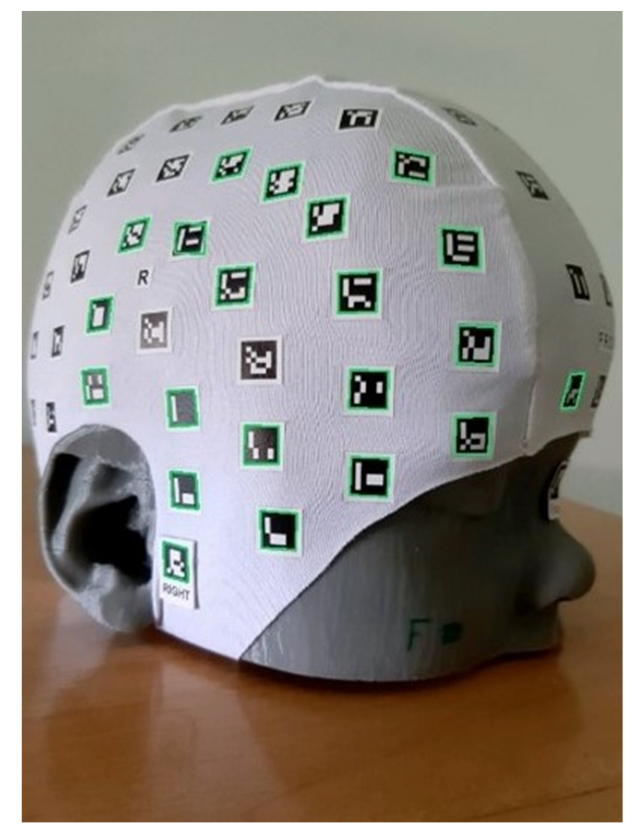

(b)

Figure 7.2: a) Coded cap and stickers; b) view of the cap on the app.

The mobile app allows data acquisition by users without understanding of the photogrammetric principles. The following criteria has been implemented to assure that any user can carry out a successful data acquisition: (i) the detected markers are highlighted in the image; (ii) the correctly registered areas are shown; and (iii) a progress bar shows the progress percentage. Each frame is checked for valuable information by the app. Firstly, it must have more than 7 markers (a lower number does not assure successful orientation). Moreover, the sets of markers in the image are evaluated to assure there is information in the image that is not already registered (a previous frame with the same set of markers would discard the current image). In case the image is selected, the coordinates of the markers are saved to a file. For each marker, the coordinates of the four corners are stored. At the same time, the app is updated to show the new registered areas on the screen. This particular approach has been designed to deal with the movement of the infant. If the patient is moving it hampers the image acquisition and no markers are detected, therefore the frames are ignored. As soon 
as the patient is still enough, even for a short period, the application will record the information. More than 200 images covering the totality of the model are required to assure a good orientation (98). Once the required number of images is registered and the whole head is correctly covered, the app will automatically send the data for processing.

\subsubsection{Model creation}

The model creation is carried out on a server. The server runs a Django (Django Software Foundation, Lawrence, KS) WSGI (Web Server Gateway Interface) application. When a model data is received the application stores the file and inserts the model metadata into a PostgreSQL database. Then, it runs the model calculation algorithm as a Celery Distributed Task Queue process and informs the user. Celery remains calculating the model as a background process in the server. The server can process several models at once. If there are many models to calculate at a time, they are put in a queue. When Celery finishes the calculation, an alert is sent to the user and the final model is made available on the webpage (Fig. 7.3).

The modelling calculation software was created using a combination of open-source software and ad-hoc developed software. First of all, the received data is checked. The frames are evaluated in order to remove redundancy from the recorded data. This step improves the selection carried out during the data acquisition. Secondly, the frames for calibration are selected. The required frames will cover the top of the cap, giving the best possible geometry for calibration. The software MicMac (110), and, specifically, the tools Tapas and AperiCloud, are used to obtain the 3D point cloud. MicMac tool Tapas is launched to carry out an on-the-job bundle adjustment camera calibration. For this camera calibration step, a reduced set of frames is selected, and if the results achieved are correct, the interior orientation parameters are extended to all the frames. Once the frames are oriented, the point cloud is then obtained using AperiCloud (Fig. 7.4 a,b). In case the bundle adjustment results are not correct, another bundle adjustment process is carried out again for the whole set of frames. MicMac was chosen as an open-source option with the possibilities to automatize the required process. The resulting point cloud consists of up to 536 points. It is scaled using the markers size and the point normals are computed automatically using MeshLab 1.3.3 


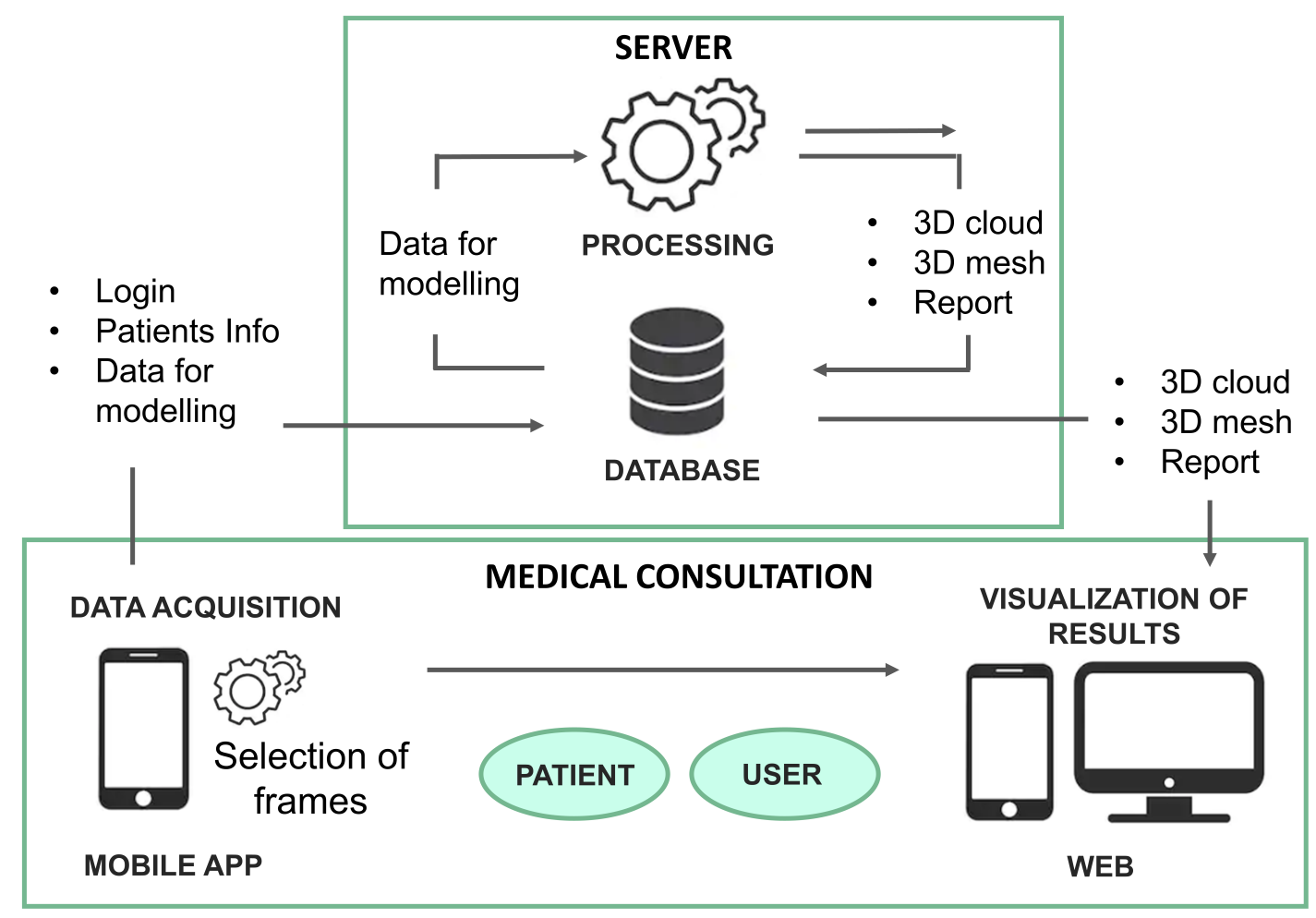

Figure 7.3: PhotoMeDAS calculation. 
(111). The mesh is also created automatically using the Ball Pivoting Algorithm (112) (see Fig. 7.4c,d).

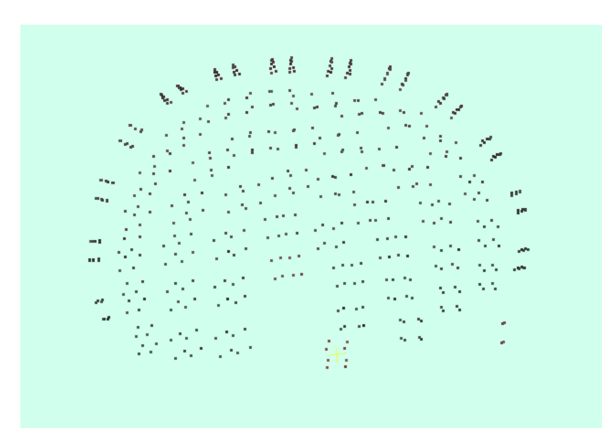

(a)

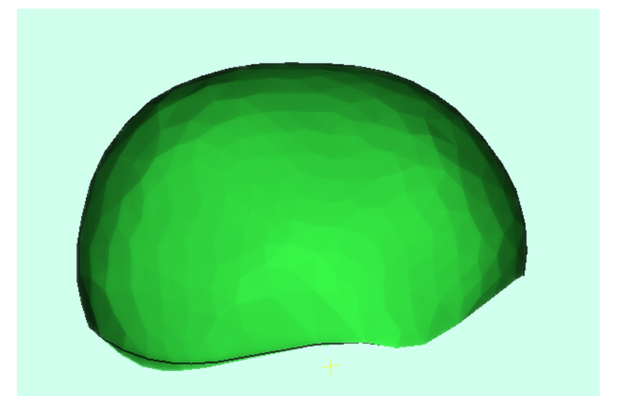

(c)

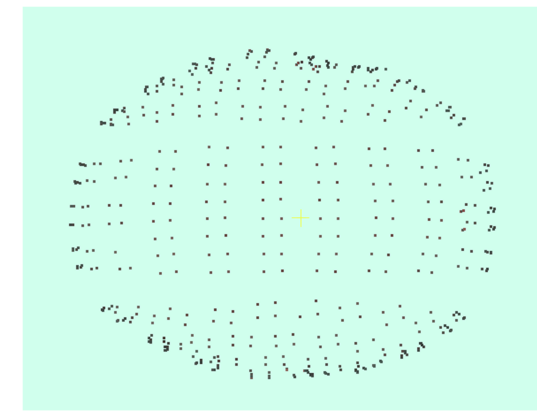

(b)

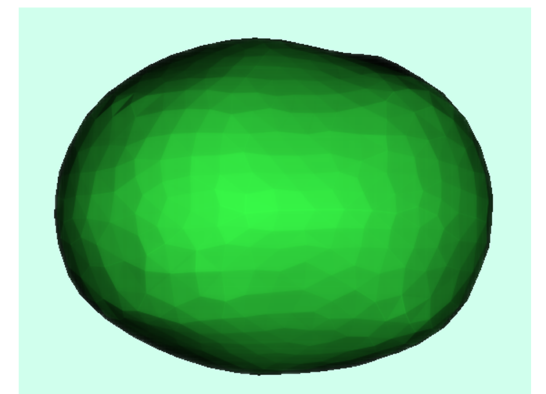

(d)

Figure 7.4: 3D point cloud of the markers (a, side view; b, top view) and mesh (c, side view; $d$, top view).

The last step is the registration of the 3D model to a set head's local coordinate system (Fig. 7.5). The three points identified with stickers are used for the 3D registration among corresponding models. The y-axis will be given by the preauricular points while the frontal point and the centre between the ears define the $\mathrm{x}$-axis. The whole process presented in this section has been integrated using an in-house Python script and it is fully automatic. The script is automatically computed every time a data file is sent to the server.

\subsubsection{Automatically-derived anthropometric linear magnitudes}

Virtual 3D models by themselves have a reduced utility for doctors. It is necessary to obtain objective parameters and indexes that give direct information on the type and degree of deformation. To obtain the automatically-derived anthropometric linear magnitudes models are registered as presented in Section 2.1.2. In this study, three 


\section{THE AUTOMATIC TOOL}

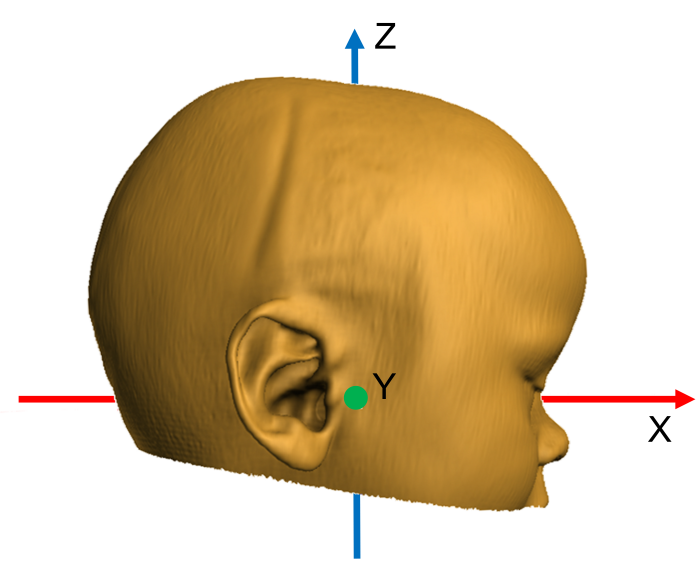

Figure 7.5: The head's local coordinate system.

basic anthropometric linear magnitudes to yield indexes are obtained for each model, maximum perimeter, maximum longitudinal distance and maximum transversal distances. The values are obtained by iterative computation in a given direction and allowing small variations to obtain the longest values. The perimeter is computed for a plane at different heights along the z-axis and different rotations in the $\mathrm{y}$-axis (Phi angle), but without rotations in the x-axis or z-axis (Omega and Kappa angles) as this orientation is given by the preauricular points. The maximum longitudinal distance is computed as the maximum distance between a point in $\mathrm{y}=0$ and located in the front of the head (front centred but at different heights) and points located in the back part of the head with same y coordinate, small rotations (maintaining the front point fixed) were allowed to deal with registration inaccuracies. The maximum transversal distance is computed along y-axis, no rotations are allowed.

\subsubsection{Validation methodology}

Five models of real infants' heads are obtained from Computed Tomography or Magnetic Resonance images. The 3D models are reconstructed using InVesalius (Centro de Tecnologia da Informação Renato Archer (CTI), Brazil). An example of the external output data is displayed in Figure 7.5. The models obtained with the gold standards imaging devices are later 3D printed using a BQ printer. Those five models will be referred to as targets (Fig. 7.6). The 3D printed head has definitely an error but it can be considered negligible in comparison with the minor fitting imperfections covered 
by the coded cap. The targets represent different types of cranial shape and deformation in infant's heads: 1) Scaphocephaly, long and narrow head caused by early fusion of sagittal suture; 2) Plagiocephaly, asymmetric distortion with a flattened area; 3) Complex deformation due to intrauterine constraint; 4) Trigonocephaly, early fusion of the metopic suture causing a triangular shape of the forehead; and 5) Normal shape considered without deformation (Fig. 7.6).

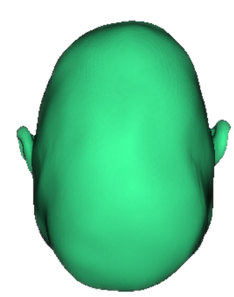

1

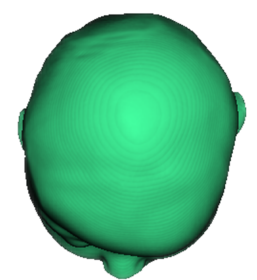

2

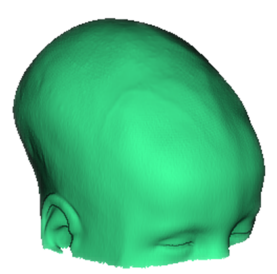

3

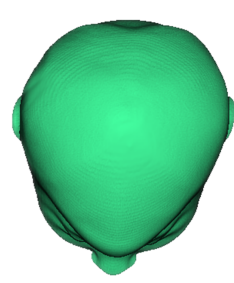

4

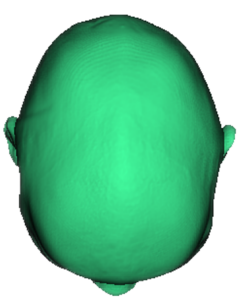

5

Figure 7.6: Targets.

Intrauser and interuser repeatability and accuracy tests are carried out for each model. For the intrauser analysis, the same person carries out 25 data acquisition per targets. For the interuser tests, 25 different people acquire the data for each target. In total 250 models (125 intrauser and 125 interuser) are created, 50 for each target. For every data acquisition, the cap and stickers are placed by the user. A short introduction (less than one minute) on the tool is given to the user, however, no further training is provided.

\subsubsection{Comparison between 3D models}

The accuracy is measured by comparing every model with the digital version of the model used for 3D printing, considered as the ground truth. The repeatability is evaluated by comparing each pair of photogrammetric 3D models. Iterative Closest Point (ICP) (39, 113, 114) algorithm is computed for each pair of models in order to improve the registration. For each pair of models, the distance is later computed along the ray from the centre of the model for each point in a sphere (Fig. 7.7). This methodology is chosen as it is proven to yield less noisy results than distances computed along the normals; besides, the provided measurements are well-distributed across the infant's head, as proposed by de Jong et al. (27). 


\section{THE AUTOMATIC TOOL}

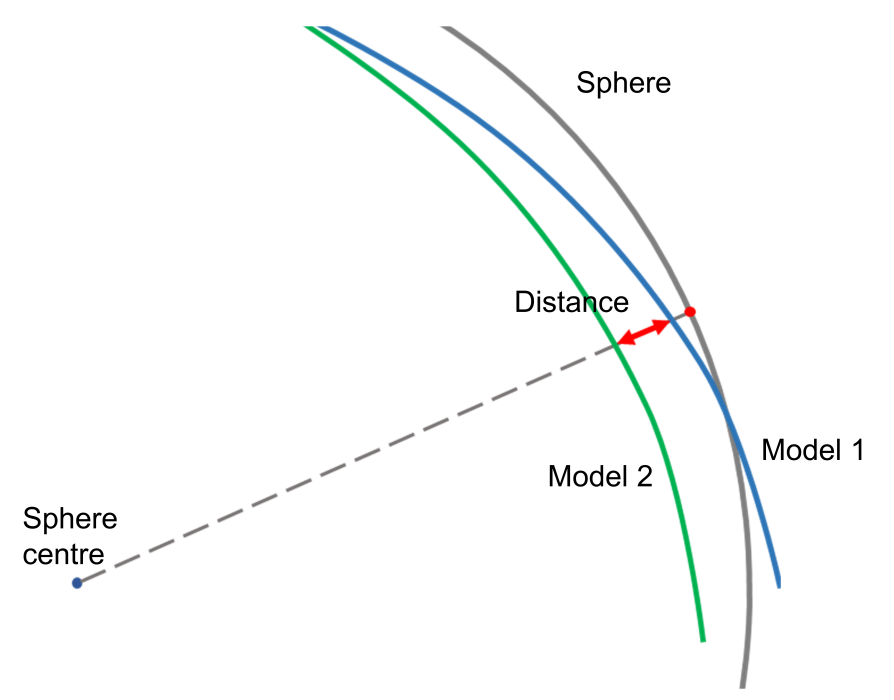

Figure 7.7: Diagram displaying the distance computed between 3D models.

\subsection{Results}

The differences between target models are presented in Figure 7.8. All tests resulted in a sum below $1 \mathrm{~mm}$ for mean plus standard deviation. The absolute differences are slightly larger for the interuser tests in comparison to those carried out by a single user (intrauser). Besides, the accuracy results are slightly worse (larger values) than the repeatability results. The mean accuracy is $0.5 \pm 0.4 \mathrm{~mm}$, and the repeatability differences are $0.3 \pm 0.3 \mathrm{~mm}$. Ideally, there should be no significant differences in the results for different tests (comparing intrauser-interuser and repeatability-accuracy). A Student t-test was used to compare the results (understood as distances between models) for different tests. The $95 \%$ confidence interval for the difference in means between repeatability and accuracy results is $0.169-0.173 \mathrm{~mm}$. For the comparison between intrauser and interuser tests, t-test $95 \%$ confidence interval of the difference in mean of the results is $0.073-0.74 \mathrm{~mm}$. Therefore, for both comparisons (intrauserinteruser and repeatability-accuracy) it can be stated that the difference in the results is below $1 \mathrm{~mm}$.

Absolute differences per model are presented in Figure 7.9. No important differences are present for Target 1-4. Slightly worse results are presented for Target 5 .

As it could be expected due to the effect of the cap, all photogrammetric 3D models showed an overestimation of measurements compared to the ground truth targets. 


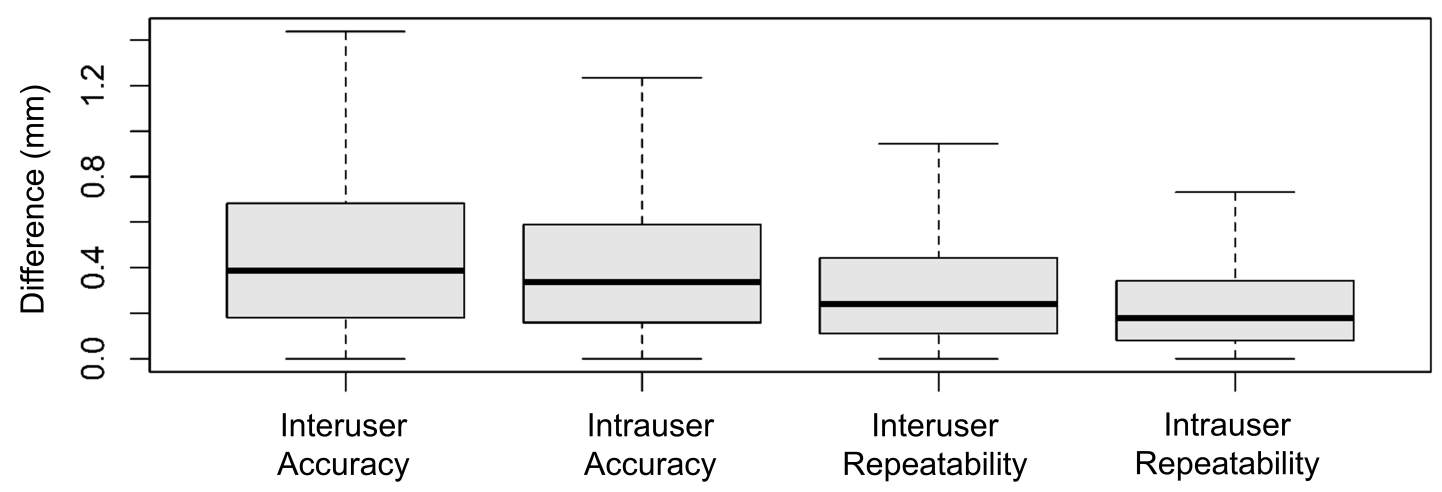

Figure 7.8: Box plots of absolute differences between models for each test.

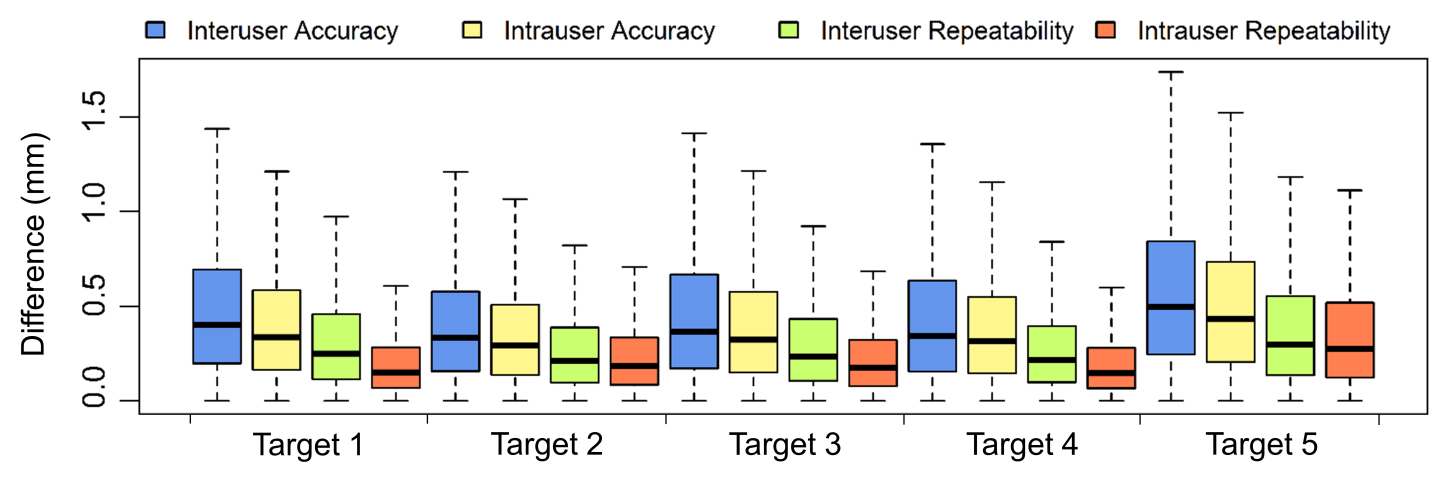

Figure 7.9: Box plot of absolute differences between models for each test and model. 


\section{THE AUTOMATIC TOOL}

Therefore, this resulted in positive distances (Fig. 7.10).

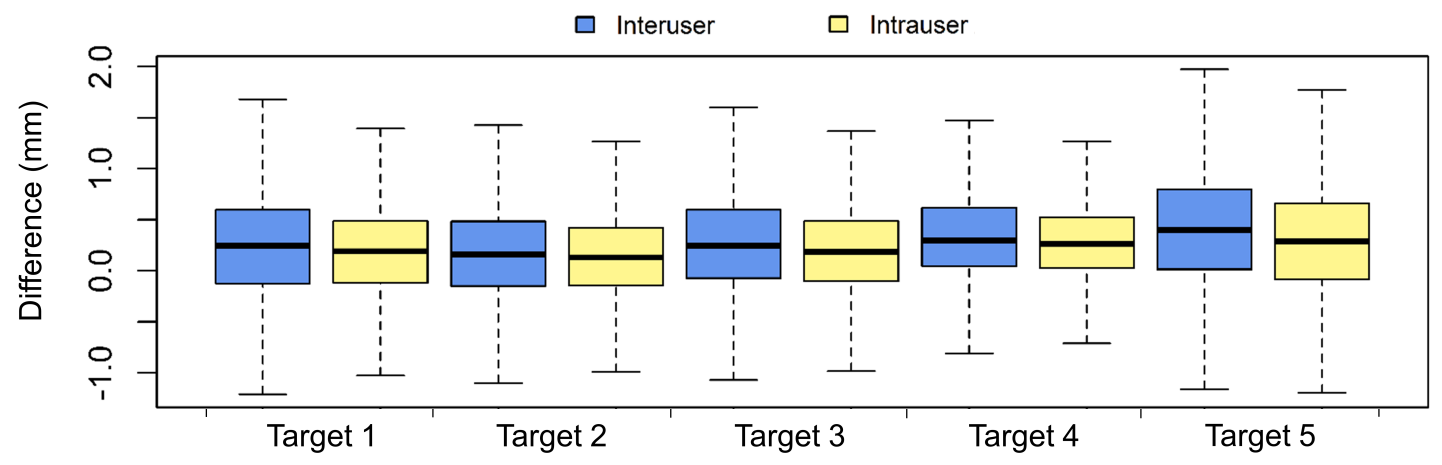

Figure 7.10: Box plot of signed differences for the accuracy tests.

The mean and standard deviation for each comparison are shown in Figure 7.11 Some models present an error considerable higher than the average, but in every case, the sum of mean and standard deviation is always below $2 \mathrm{~mm}$.

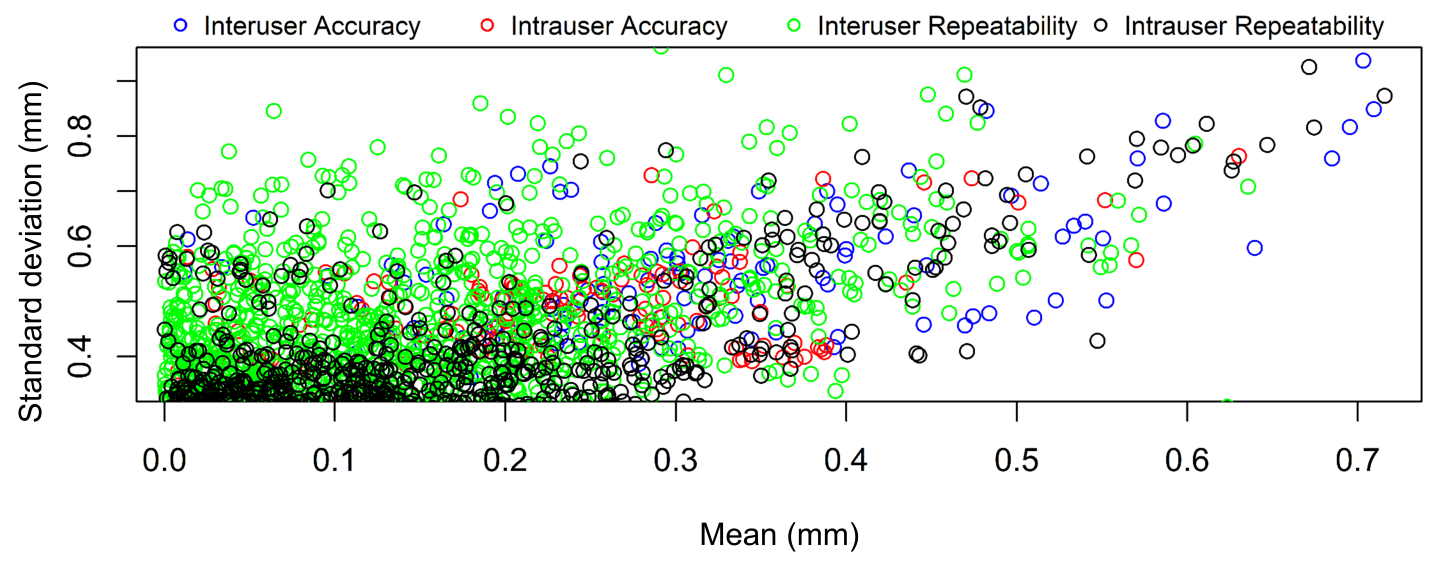

Figure 7.11: Mean and standard deviation for each created model.

Data acquisition and calculation time were registered for each created model. For the models created by a single user familiar with the methodology, the acquisition time was $1.1 \pm 0.17$ minutes. For various users, the acquisition time was higher: $1.5 \pm 0.6$ minutes. The calculation time was $2.5 \pm 0.6$ minutes for the expert user and $2.7 \pm 0.9$ minutes for different users, many of them with no previous knowledge of the tool. For the five targets, the variability of the three most important anthropometric linear magnitudes is presented: perimeter, longitudinal and transversal distances. A Student's 
t-test was also performed to evaluate the significance of the difference in mean between intrauser and interuser tests. A second t-test was performed to test the hypothesis of the differences in means being greater than $1 \mathrm{~mm}$. Table 7.1 summarises the results achieved from the automatically-derived anthropometric linear magnitudes for each target.

The measurement of the perimeter yields the lowest reliability with a difference with the mean of $1.4 \pm 1.3 \mathrm{~mm}$. For the maximum distances, the differences are $0.7 \pm 0.6 \mathrm{~mm}$ for the longitudinal distance and $0.5 \pm 0.5 \mathrm{~mm}$ for the transversal distances. Student's t-test is performed to evaluate the differences in the means between intrauser and interuser measurements. A P-value below 0.05 is interpreted as a significant difference between the means. According to this threshold, some anthropometric magnitudes (all for Target 3, the perimeter for Target 4 and the perimeter and the longitudinal distance for Target 5), show significant differences for intrauser and interuser tests. Although significant, the difference values are low, and only for the Target 3 perimeter can be stated that the mean difference between interuser and intrauser value is significantly greater than $1 \mathrm{~mm}$.

\subsection{Discussion}

In this section, we discuss the advantages and limitations of the created photogrammetric tool and its applicability for cranial deformation assessment. The results are evaluated in two different ways: the accuracy achieved in the targets (3D models) and the accuracy of the automatically-derived anthropometric linear magnitudes. The factors that limit the accuracy of the results are also studied, and future lines of research for the improvement of the solution are presented.

\subsubsection{The automatic tool}

A fully automatic tool for the creation of head 3D models has been presented. The tool is adapted to the necessities of cranial deformation assessment in infants. In particular, it overcomes limitations, such as the almost constant movement of the infants during the data acquisition, it requires very low investment (from the user side only a smartphone is required) and does not require the user to have any knowledge of photogrammetry, being applicable to the standard clinical routine. The designed methodology, based on 


\begin{tabular}{|c|c|c|c|c|c|c|c|}
\hline & & & Mean & SD & $95 \mathrm{CI}$ & $\mathrm{P}$ & P1 \\
\hline \multirow{6}{*}{ Target 1} & \multirow{2}{*}{ Perimeter } & Intrauser & 412,2 & 0,9 & $411,9-412,6$ & \multirow[t]{2}{*}{0,11} & \multirow[t]{2}{*}{0,59} \\
\hline & & Interuser & 413,1 & 2,4 & $412-414,1$ & & \\
\hline & \multirow{2}{*}{ Longitudinal distance } & Intrauser & 151,7 & 0,4 & $151,5-151,9$ & \multirow{2}{*}{0,06} & \multirow{2}{*}{0,97} \\
\hline & & Interuser & 152,2 & 1,2 & $151,7-152,7$ & & \\
\hline & \multirow{2}{*}{ Transversal distance } & Intrauser & 104,3 & 0,5 & $104-104,5$ & \multirow{2}{*}{0,17} & \multirow{2}{*}{0,99} \\
\hline & & Interuser & 104,6 & 0,9 & $104,2-104,9$ & & \\
\hline \multirow{6}{*}{ Target 2} & \multirow{2}{*}{ Perimeter } & Intrauser & 377,8 & 1,5 & $377,1-378,4$ & \multirow{2}{*}{0,4} & \multirow{2}{*}{0,85} \\
\hline & & Interuser & 378,2 & 2,1 & $377,3-379,1$ & & \\
\hline & \multirow{2}{*}{ Longitudinal distance } & Intrauser & 127,3 & 0,6 & $127-127,5$ & \multirow{2}{*}{0,62} & \multirow{2}{*}{1} \\
\hline & & Interuser & 127,4 & 1,1 & $126,9-127,9$ & & \\
\hline & \multirow{2}{*}{ Transversal distance } & Intrauser & 110,9 & 0,5 & $110,7-111,1$ & \multirow{2}{*}{0,38} & \multirow{2}{*}{1} \\
\hline & & Interuser & 111,0 & 0,7 & $110,8-111,3$ & & \\
\hline \multirow{6}{*}{ Target 3} & \multirow{2}{*}{ Perimeter } & Intrauser & 430,1 & 1,0 & $429,6-430,5$ & \multirow{2}{*}{$<0.001$} & \multirow{2}{*}{0,03} \\
\hline & & Interuser & 432,0 & 2,1 & $431,1-432,9$ & & \\
\hline & \multirow{2}{*}{ Longitudinal distance } & Intrauser & 151,6 & 0,8 & $151,2-151,9$ & \multirow{2}{*}{$<0,001$} & \multirow{2}{*}{0,21} \\
\hline & & Interuser & 152,8 & 1,0 & $152,4-153,2$ & & \\
\hline & \multirow{2}{*}{ Transversal distance } & Intrauser & 117,2 & 0,6 & $117-117,5$ & \multirow{2}{*}{0,019} & \multirow{2}{*}{1} \\
\hline & & Interuser & 117,7 & 0,6 & $117,4-117,9$ & & \\
\hline & Porimeter & Intrauser & 367,9 & 1,4 & $367,3-368,5$ & 0.024 & 036 \\
\hline & Ferimeter & Interuser & 369,1 & 2,0 & $368,2-370$ & 0,024 & 0,50 \\
\hline Target 4 & Lonoitudinal distance & Intrauser & 126,9 & 0,6 & $126,6-127,1$ & 0.099 & 1 \\
\hline 1arget 4 & Longituamal distance & Interuser & 127,2 & 0,9 & $126,8-127,6$ & 0,099 & 1 \\
\hline & & Intrauser & 111,2 & 0,4 & $111-111,3$ & & \\
\hline & Iransversal distance & Interuser & 111,6 & 1,0 & $111,2-112$ & 0,002 & 1 \\
\hline & Perimeter & Intrauser & 451,3 & 2,2 & $450,4-452,2$ & $000 ?$ & 0.05 \\
\hline & Fermimeter & Interuser & 453,3 & 2,1 & $452,4-454,2$ & 0,002 & 0,05 \\
\hline Toroet 5 & Innoitudingl dictan & Intrauser & 160,1 & 0,7 & $159,8-160,4$ & & 0.42 \\
\hline Iarget o s a s a & Lomblutumal distante & Interuser & 161,2 & 1,0 & $160,7-161,6$ & $<0,001$ & 0,42 \\
\hline & $\operatorname{Tr}$ & Intrauser & 122,7 & 1,0 & $122,3-123,2$ & 0125 & 0.06 \\
\hline & 11alisvers & Interuser & 123,2 & 1,0 & $122,8-123,6$ & 0,120 & 0,00 \\
\hline
\end{tabular}

Table 7.1: Mean and standard deviation (SD), Student's t-test $95 \%$ confidence interval (95 CI), P-value (P) for comparison of the means between intrauser and interuser values, and $\mathrm{P}$ value for the hypothesis of an absolute true difference in mean $<1 \mathrm{~mm}(\mathrm{P} 1)$ (Values in $\mathrm{mm})$.

markers, has very low requirements of data storage and transference. This is especially useful as the data requires to be sent to the servers during the consultation. Not all hospitals and clinical centres have high-speed networks. The average acquisition and 


\subsection{Discussion}

processing time was 3.9 minutes, while the longest time was 7.6 minutes. This processing time means a huge improvement in comparison with the manual photogrammetric process, that can require hours of expert work to achieve an accurate model (61). This processing workflow makes the tool viable in normal clinical practice. One advantage is that once the user gets familiar with the photogrammetric tool, the processing time goes down, as reflected with the different between intrauser and interuser tests.

\subsubsection{D models}

A final accuracy of $1.5 \mathrm{~mm}$ was achieved. The purpose of the research project was not to deliver the highest possible accuracy but reliable measurements for doctors with real-life available devices, i.e. smartphones. From a photogrammetric standpoint, this result could be considered somehow above the required standards for cranial deformation assessment. However, medical staff suggest that facial asymmetry below $2 \mathrm{~mm}$ cannot be noticed by visual assessment (115). Therefore, the data provided by the PhotoMeDAS tool is enough to assess esthetical outcomes for infant's heads. Significant differences were obtained for intrauser and interuser tests according to Student's t test. However, the differences in means were always below $1 \mathrm{~mm}$ for a $95 \%$ confidence interval. As a consequence, we can conclude that the accuracy of the smartphonebased photogrammetric solution is not greatly affected by different non-expert users. The distribution of the error areas is especially important. Distances above $1 \mathrm{~mm}$ were registered in the repeatability tests in the edge of the 3D models. In these areas, the models are not as accurate due to the geometry of the image acquisition. However, the areas of inaccuracy are small enough to be ignored for deformation assessment. Comparing the deliverables from PhotoMeDAS with the ground truth 3D models (five targets), a general overestimation is appreciated. One particular issue can be found in convex areas where higher differences (distances, Fig. 7.7) can be achieved (e.g. Target 1). This error is given by the impossibility to assure that the cap is stuck to the infant's skin in these areas (Fig. 7.12).

Previous tests were carried out by the authors using a manual approach for the creation of the 3D models (98). The results of the study show that the accuracy of the automatic tool is very similar to the manual approach carried out by photogrammetric experts to create the 3D models. The difference between repeatability and accuracy tests results shows that the highest differences are given by the impossibility to properly 


\section{THE AUTOMATIC TOOL}

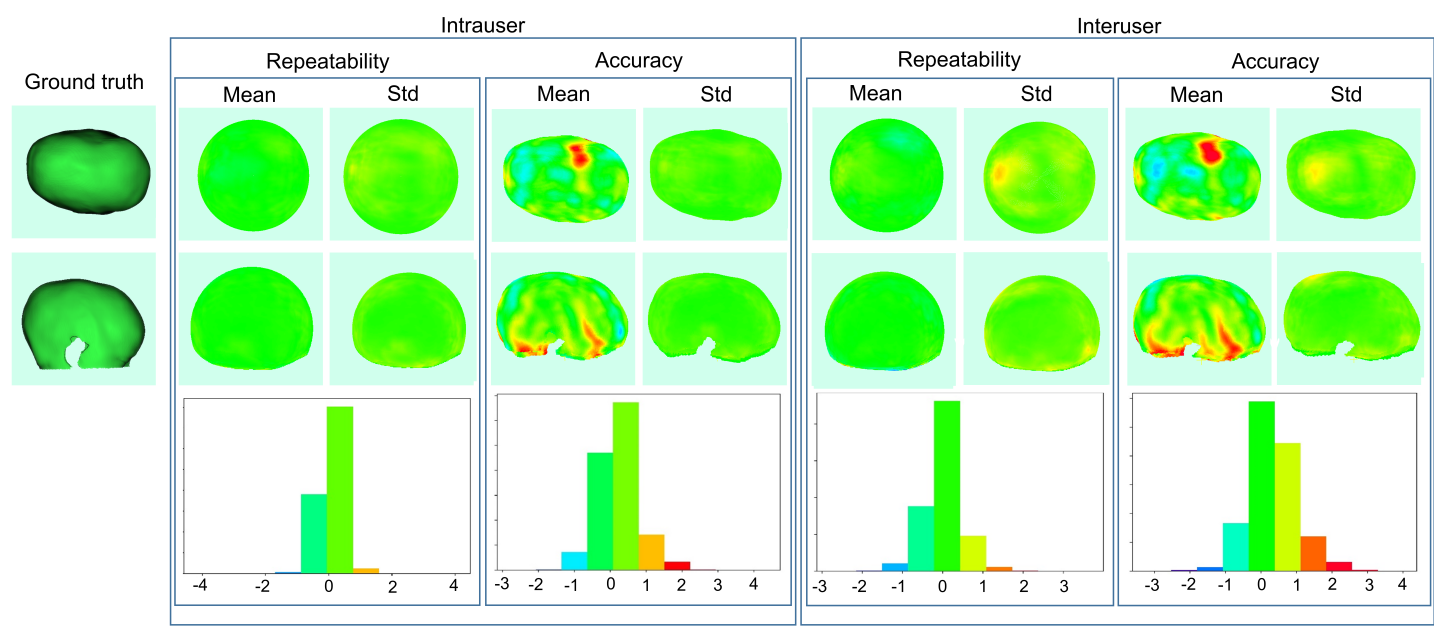

Figure 7.12: Representation of distances for Target 1. For the repeatability tests, the distances are shown on a reference sphere with its centre in the model centroid. For the accuracy tests, the distances are shown on the ground truth model. Histograms for each test are shown at the bottom (values in $\mathrm{mm}$ ).

fit the cap to the convex areas. However, these convex areas usually represent a small area of the head and do not compromise the evaluation of the general infant's head shape. The tool provides 3D models generated from a total of 536 points, which are later interpolated to create a model. According to the results, this quantity of points can be considered enough for accurate infant's head shape representation.

\subsubsection{Automatically-derived anthropometric linear magnitudes}

The automatic anthropometric linear magnitudes show acceptable accuracy for the maximum longitudinal and transversal distances with standard deviation up to 1.17 $\mathrm{mm}$. For the perimeter measurements, standard deviations rise above $2 \mathrm{~mm}$ in some cases. For the perimeter measurements and even for longitudinal and transversal distances in some cases, the registered accuracy is worse than the accuracy of the models. Three main reasons have been identified as possible causes of the limited accuracy of the anthropometric linear magnitudes. 1) Firstly, the level of accuracy of the photogrammetric model is an important source of error. The correlation between the accuracy of the photogrammetric models and the accuracy of the measurements (as a difference with the average) was calculated for each model and for the automatic anthropometric linear magnitudes. For every case the correlation was negligible. The correlation was 
also calculated for the intrauser models only with very similar results. The lack of correlation implies that, although it can be important, the quality of the photogrammetric models is not the main factor affecting the accuracy of the automatically derived anthropometric linear magnitudes. 2) The second source of error considered is the variability in the placement of the cap. This explains the significant differences between interuser and intrauser tests for the perimeter values according to the Student's t-test. A single user is supposed to have much lower variability in the process of cap and sticker placement. Differences in the placement of the cap greatly affect the obtainment of the measurements, in some cases, important differences were detected when the cap was not covering the whole forehead of the patient (Fig. 7.13).

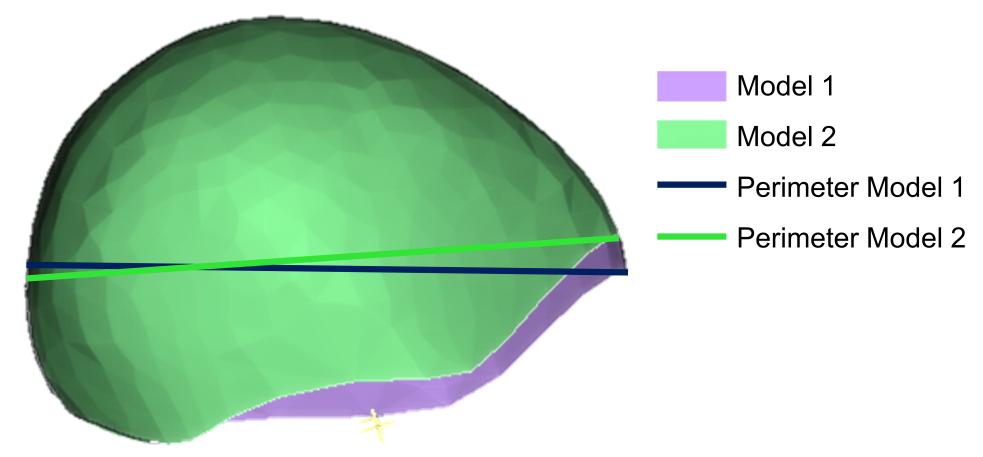

Figure 7.13: Comparison of perimeter measurements between two models of the same Target.

3) Lastly, the registration of the model, based on the placement of the coded stickers has an implied variability. This can result in slightly different measurements even for the same model. It could also be explaining the differences (below $1 \mathrm{~mm}$ for longitudinal and transversal distances but above that, in some cases, for the perimeter) between interuser and intrauser tests. The correct automatic registration of the models is a common challenge to many other methodologies used for cranial deformation analysis (103). Correct placement of the cap and stickers is vital for the tool to provide useful data. However, the $3 \mathrm{D}$ model, together with the measurement points, are made available to the doctor in a short time frame after the data acquisition. Therefore, an error in the obtainment of the derived anthropometric linear magnitudes caused by misplacement 


\section{THE AUTOMATIC TOOL}

of the cap or the stickers, could be easily noted by the doctor, who could discard the measurement and repeat the data acquisition (if required).

\subsubsection{Comparison with current solutions}

The results provide higher accuracy than to those obtained with measuring tape and calliper (20), especially for transversal and longitudinal distances. Moreover, the interuser reliability of the photogrammetric 3D models seems to be higher for this tool and the models provide much more complete information, as the whole infant's head is measured. The CT and MRI, considered the gold standards, provide the highest accuracy. However, its use tends to be limited as they are costly and, more importantly, often required sedation and even imply radiation. Moreover, the lying position of the infants during radiologic image acquisition is not ideal for the assessment of the esthetical outcome of the patients. The methodology is comparable in accuracy to low-cost scanners that are used on static targets only, such as Vectra H1 system (Canfield Imaging, NJ, USA) (116). It provides also a much more complete information than other low-cost techniques that rely on a small number of images (117). Last but not least, high-end image-based and range-based solutions including several cameras and scanners provide higher accuracy than the presented tool (25, 118). However, its present use is limited to a few clinics due to the high related cost (not only in the metrical device but maintenance, personnel, etc.). Moreover, for the particular application of cranial deformation assessment, submillimeter accuracy presents little or meaningless advantage.

\subsection{Conclusion}

The presented photogrammetric solution has been proved to be valuable for the automatic achievement of infants' head 3D models. As during a normal medical consultation, unpredicted quick infant's movements exist and it is unrealistic to carry out comprehensive intrauser and interuser tests. The analysis has been undertaken with five true 3D printed infant's head targets to assess the implemented methodology adopted in PhotoMeDAS. The obtained accuracy is higher to commonly used methodologies such as calliper and measuring tape but it provides more complete information. Due to is low-cost, ease of use and reduced processing time, it is expected to be integrated as part 
of the clinical routine. Contrary to high-cost image-based and range-based devices, the PhotoMeDAS implementation can be available at primary care consultations, allowing a higher percentage of infants to be objectively tested at early stages, contributing to better outcomes and diagnosis. PhotoMeDAS can improve the assessment of an important problem such as infant's cranial deformation, without following the highly subjective manual measurements with callipers, and avoiding radiologic imaging. In the near future, PhotoMeDAS will be clinically validated from a medical point of view on real infant's heads to assure whether it can be considered a medical device or not. Future developments will include the automatic extraction of cranial indexes as well and the improvement in the registration of the infant's head 3D models, as this has been identified in this study to be one of the major sources of metric differences. 


\section{8}

\section{Overall Discussion}

Cranial deformation is a major concern for parents, paediatricians and craniofacial surgeons. It is necessary to accurately measure the cranial shape of the infants to detect and assess deformation, as well as to assure the best possible evolution and outcomes of the different treatments (9, 10). In this study, the possibilities of photogrammetry as a non-invasive tool for cranial deformation analysis have been studied. A new tool, designed to measure cranial deformation during the routine clinical consultation has been developed.

The first step of the study consisted of a test to evaluate the possibilities of the creation of head 3D models using images. This step was presented in Chapter 2. The models were created using an SLR camera and manual processing. To evaluate the accuracy of the results manual measurements carried out by experts were compared with measurements extracted from the 3D model. The differences between both methodologies were found to be very dependent on the correct identification of craniofacial points. When the measurement points were correctly identified, the differences between methodologies were up to 2 to $3 \mathrm{~mm}$ depending on the measurement. The accuracy of the models was therefore considered acceptable for cranial deformation assessment. It was also pointed out that the models provide more detailed information than manual measurements, as they represent the totality of the head (61). The main problem of the methodology was found to be the acquisition step. The processing is required to be quick and automatic in order to be applied in real clinical conditions. The automation procedures started with the use of a smartphone camera, as it was presented in Chapter 3. During this step of the development a smartphone was used 
for image acquisition. The smartphone, and, specifically the slow-motion camera mode was found to be of great utility to acquire data even with patients in movement. The instability of smartphone cameras was a challenge but results show differences below 1 mm compared to models obtained using SLR cameras (97). In order to obtain a useful tool, it was necessary to make it automatic and assure its reliability. The determination of the best methodology for data acquisition and processing was presented in Chapter 4. The importance of different factors for the creation of the $3 \mathrm{D}$ models was evaluated. A high hyper-redundancy was found to be crucial to achieve accurate models. A network consisting of 3 rings of images around the patient's head was found to be the optimal geometry. The calibration method was found to be of low importance as long as a high number of images were acquired. Apart from the number of images, the factor affecting the most the quality of the models was the existence of easily detectable markers. At this point, an error of $0.2 \mathrm{~mm}$ was found to be achievable under ideal conditions (98). Based on the previous conclusions a semiautomatic tool was presented and evaluated in Chapter 5. A special cap covered with coded targets was used and the data acquisition was carried out using a smartphone. The tool was evaluated in real clinical conditions, and the results were compared with the gold standard, CT and MRI (102). An overestimation of the photogrammetric models above $3 \mathrm{~mm}$ was obtained in some cases. However, the standard deviation of the distances between the models was $1.5 \mathrm{~mm}$. The results were considered acceptable as differences in shape (given by the standard deviation) were below the required standards for cranial deformation analysis (115). Some of the differences were explained by inaccuracies of the photogrammetric models while some part of the error was attributed to the radiological tests and the differences in the position of the patients, the effect of the cap, etc. As a consequence, the error of the photogrammetric process is considered to be lower than the difference between both methodologies. The obtainment of objective indexes from the model is required in order to make the tool useful for doctors. In order to obtain objective measurements from the models, a registration methodology was developed (119) and it was presented in Chapter 6. The use of three manually identified points has been proved to be adequate to the necessities of the tool and provided an accuracy of $3 \mathrm{~mm}$ (119). All the conclusions obtained in previous studies were integrated to create an automatic tool (120) presented in Chapter 7. A smartphone app guides the user to acquire the information in the best possible way, following the requirements 


\section{OVERALL DISCUSSION}

stated by previous studies (number of images, distribution and markers). The data is later processed in a server to create a model. The models are registered and automatic measurements are obtained. The tool was evaluated using 3D printed head models and the data was taken by different users. The results showed an accuracy of the models of $1.5 \mathrm{~mm}$. This error is considerably higher than the differences found for the models in ideal conditions test carried out in the lab. The difference is explained by the limitations of the automatic tool that cannot assure the optimal image acquisition in all cases and users and also by the use of targets instead of images for the creation of the models, resulting in models with a much lower number of points. These limitations were justified by the low computational cost and the low quantity of data that needs to be stored. Despite this, the accuracy is considered adequate for the application. The automatic measurements showed accuracy above the results reported by calliper and measuring tape for some authors (20). Perimeter accuracy was up to $2 \mathrm{~mm}$ and longitudinal and transversal measurements were below to $1.5 \mathrm{~mm}$. The mean time of acquisition and processing was below 4 minutes (120). The results obtained by the tool have proven to provide more information and be more accurate than currently used, low-cost methodologies such as calliper and measuring tapes. The results are comparable to the gold standards, given by CT and MRI. Although the accuracy is lower, the presented methodology has the important advantage of being non-invasive, and the resulting accuracy perfectly allows proper clinical evaluation of the cranial deformation. In comparison with other image-based and range-based methodologies, the accuracy of the results is slightly worse (120), but the cost of the tool is remarkably lower and it does not require experts to carry out the data acquisition. 


\section{9}

\section{Conclusions}

This project presents a solution to a problem addressed by paediatric neurosurgeons. There is a need for accurate, non-invasive and objective methodologies to measure cranial deformation in infants. It is important to improve the early detection of the deformation and assess the evolution of the patients after different treatments. The goal of the study was to develop a tool that would meet the needs the non-photogrammetric experts and would be suitable for inclusion in routine medical consultation. It can be concluded that the presented photogrammetric tool is a powerful enough tool to extract valuable measurements of infants' heads. The presented tool has achieved the goal of being totally automatic, low-cost and non-invasive and provide whole-head information. Real-life tests have shown that it can be used in clinics by users with little experience. The methodology provides useful and objective measurements of the infants' head. We consider the methodology to achieve the required accuracy while maintaining the minimum costs and requirements. Important steps have been carried out to obtain indexes and measurements that can help doctors during evaluation and decision-making. However, there is still a long path in the development of model-based, more detailed measurements that provide better information and represent the deformation more accurately. Some of these future development paths have been mentioned, as the comparison to an ideal head. The acquisition of bigger quantities of highly detailed data could lead to the development of algorithms for a better understanding of morphological evolution in children. The methodology, as a simple and low-cost method to extract 3D models of moving targets might be in the future applied to other applications in medicine or other fields. 


\section{References}

[1] W. K. Peitsch, C. H. Keefer, R. A. Labrie, and J. B. MulLIKEN. Incidence of Cranial Asymmetry in Healthy Newborns PEDIATRICS, 110[6]:e72-e72, 2004. 1] 2

[2] Aliyah Mawji, Ardene Robinson Vollman, Jennifer Hatfield, Deborah A. McNeil, and Reginald Sauvé. The incidence of positional plagiocephaly: A cohort study. Pediatrics, 132[2]:298-304, aug 2013. 1

[3] Leslie G. Branch, Kendra Kesty, Elizabeth Krebs, LindSey Wright, Stephanie Leger, and Lisa R. David. Deformational Plagiocephaly and Craniosynostosis: Trends in Diagnosis and Treatment After the "Back to Sleep" Campaign. The Journal of Craniofacial Surgery, 26[1]:147-150, 2015. 12

[4] Yahya Khormi, Michelle Chiu, Ronette Goodluck Tyndall, Patricia Mortenson, David Smith, and Paul Steinвок. Safety and efficacy of independent allied healthcare professionals in the assessment and management of plagiocephaly patients. Child's Nervous System, 36[2]:373-377, feb 2020. 14

[5] Andrea E Bialocerkowski, Sharon L Vladusic, and Choong Wei NG. Prevalence, risk factors, and natural history of positional plagiocephaly: a systematic review Developmental Medicine \&6 Child Neurology, 50[8]:577-586, aug 2008. 1]

[6] J. Kattwinked, J. G. Brooks, M. E. Keenan, M. Malloy, M. Willinger, and N. J. Scheers. Changing concepts of sudden infant death syndrome: Implications for infant sleeping environment and sleep position, mar 2000. 2

7] Brent R Collett, Erin R Wallace, Deborah Kartin, Michael L Cunningham, and Matthew L Speltz. Cognitive outcomes and positional plagiocephaly $\mathrm{Pe}$ diatrics, 143[2]:e20182373, feb 2019. 2

[8] Alexandra L.C. Martiniuk, Cassandra Vujovich-Dunn, Miles Park, William Yu, and Barbara R. Lucas. Plagiocephaly and Developmental Delay: A Systematic Review, jan 2017. 2

[9] Kevin M. Kelly, Timothy R. Littlefield, Jeanne K. Pomatto, Catherine E. Ripley, Stephen P. Beals, and EdWARD F. JOGANIC. Importance of Early Recognition and Treatment of Deformational Plagiocephaly with Orthotic Cranioplasty The Cleft Palate-Craniofacial Journal, 36[2]:127-130, mar 1999. 274,100
[10] I. Cabrera-Martos, M. C. Valenza, A. Benítez-Feliponi, C. Robles-Vizcaíno, A. Ruiz-Extremera, and G. ValenzaDEMET. Clinical profile and evolution of infants with deformational plagiocephaly included in a conservative treatment program. Child's Nervous System, 29[10]:1893-1898, oct 2013. 2100

[11] Joseph E. Losee, A. Corde Mason, Jason Dudas, Lan B HUA, AND MARK P. MOONEY. Nonsynostotic occipital plagiocephaly: Factors impacting onset, treatment, and outcomes may 2007. 2

[12] David Johnson and Andrew O M Wilkie. Craniosynostosis European Journal of Human Genetics, 19[4]:369376, apr 2011. 2

[13] William M Weathers, David Khechoyan, Erik M WolfSWinkel, Kriti Mohan, ANdRew Nagy, Robert J Bollo, EdWard P Buchanan, LaRry H Hollier, AND JR. A novel quantitative method for evaluating surgical outcomes in craniosynostosis: pilot analysis for metopic synostosis. Craniomaxillofacial trauma E reconstruction, $\mathbf{7}[1]: 1-8, \operatorname{mar} 2014$. 2

[14] Martijn Cornelissen, Bianca den Ottelander, Dimitris Rizopoulos, René van der Hulst, Aebele MinK van der Molen, Chantal van der Horst, Hans Delye, Marie-Lise van Veelen, Gouke Bonsel, and Irene Mathijssen. Increase of prevalence of craniosynostosis. Journal of Cranio-Maxillofacial Surgery, pages 1-7, 2016. 2

[15] F Ursitti, T Fadda, L Papetti, M Pagnoni, F Nicita, G IanNETTI, AND A SPALICE. Evaluation and management of nonsyndromic craniosynostosis Acta Paediatrica, 100[9]:1185-1194, sep 2011. 2

[16] Louis Argenta. Clinical Classification of Positional Plagiocephaly Journal of Craniofacial Surgery, 15[3]:368-372, may 2004. 3

[17] Jan-Falco Wilbrand, Martina Wilbrand, Joern Pons-Kuehnemann, Joerg-Christoph Blecher, Petros Christophis, Hans-Peter Howaldt, and Heidrun SCHAAF. Value and reliability of anthropometric measurements of cranial deformity in early childhood. Journal of cranio-maxillo-facial surgery official publication of the European Association for Cranio-Maxillo-Facial Surgery, 39[1]:24-9, jan 2011. 3

[18] Mette Hobaek Siegenthaler. Methods to Diagnose, Classify, and Monitor Infantile Deformational Plagiocephaly and Brachycephaly: A Narrative Review Journal of Chiropractic Medicine, 14[3]:191204, 2015. 34 4

[19] Leslie G Farkas and Stephen A Schendel. Anthropometry of the Head and Face. American Journal of Orthodontics and Dentofacial Orthopedics, 107[1]:112-112, 1995. [3]

[20] Patricia A Mortenson and Paul Steinbok. Quantifying positional plagiocephaly: Reliability and validity of anthropometric measurements Journal of Craniofacial Surgery, 17[3]:413-419, may 2006. 373 $9 8 \longdiv { 1 0 2 }$

[21] Julielynn Y. Wong, Albert K. Oh, Eitchi Ohta, Anne T. Hunt, Gary F. Rogers, John B. Mulliken, and Curtis K. Deutsch. Validity and reliability of craniofacial anthropometric measurement of $3 \mathrm{D}$ digital photogrammetric images. Cleft Palate-Craniofacial Journal, 45[3]:232-239, 2008. 3 
22] Kyra E. Stull, Meredith L. Tise, Zabiullah Ali, and DAVID R. FOWLER. Accuracy and reliability of measurements obtained from computed tomography 3D volume rendered images Forensic Science International, 238:133-140, 2014. 3

[23] Jodi M. Caple, Carl N. Stephan, laura S. Gregory, and Donna M. MacGregor. Effect of Head Position on Facial Soft Tissue Depth Measurements Obtained Using Computed Tomography Journal of Forensic Sciences, 61[1]:147-152, jan 2016. 3

24] Lachlan Munn and Carl N. Stephan. Changes in face topography from supine-to-upright position-And soft tissue correction values for craniofacial identification. Forensic Science International, 289:40-50, aug 2018. 3

[25] Heinz-Theo Lübbers, Laurent Medinger, Astrid Kruse, Klaus Wilhelm Grätz, and Felix Matthews. Precision and Accuracy of the 3dMD Photogrammetric System in Craniomaxillofacial Application Journal of Craniofacial Surgery, 21[3]:763-767, may 2010. 3 498

[26] J. W. Meulstee, L. M. Verhamme, W. A. Borstlap, F. Van Der Heijden, G. A. De Jong, T. Xi, S. J. Bergé, H. DeLYE, AND T. J.J. MAAL. A new method for threedimensional evaluation of the cranial shape and the automatic identification of craniosynostosis using 3D stereophotogrammetry Internationa Journal of Oral and Maxillofacial Surgery, 46[7]:819826 , jul 2017. 46162

[27] Guido de Jong, Manon Tolhuisen, Jene Meulstee, Ferd van der Heijden, Erik van Lindert, Wilfred Borstlap, Thomas MaAl, and Hans Delye. Radiation-free 3D head shape and volume evaluation after endoscopically assisted strip craniectomy followed by helmet therapy for trigonocephaly Journal of Cranio-Maxillofacial Surgery, 45[5]:661-671, may 2017. 489

28] Chin Kai Hsu, Rami R. Hallac, Rafael Denadai, Sheng We Wang, Alex A. Kane, Lun Jou Lo, and Pang Yun Chou. Quantifying normal head form and craniofacial asymmetry of elementary school students in Taiwan. Journal of Plastic, Reconstructive and Aesthetic Surgery, 72[12]:2033-2040, dec 2019. 4

[29] Klaus W.W. Dörhage, Jörg Wiltfang, Vera von Grabe, Annalfna Sonntag, Stephan T Becker, and Benedicta $\mathrm{E}$. BECK-BROICHSITTER. Effect of head orthoses on skull deformities in positional plagiocephaly: Evaluation of a 3-dimensional approach Journal of Cranio-Maxillofacial Surgery, mar 2018. 4

[30] H. L. Mitchell and I. Newton. Medical photogrammetric measurement: Overview and prospects. ISPRS Journal of Photogrammetry and Remote Sensing, 56[5-6]:286-294, aug 2002. 4

31] Petros Patias. Medical imaging challenges photogrammetry. ISPRS Journal of Photogrammetry and Remote Sensing, 56[5-6]:295-310, aug 2002. 4

32] Petra Urbanová, Petr Hejna, and Mikoláš Jurda. Testing photogrammetry-based techniques for threedimensional surface documentation in forensic pathology. Forensic Science International, 250:77-86, may 2015. 4
[33] Halina Ey-Chmielewska and Matgorzata Fraczak Bogumita Chrú́ciel-Nogalska. Photogrammetry and Its Potential Application. Advances in clinical and experimental medicine: official organ Wroclaw Medical University, 24[4]:737-741, 2015. 4]

[34] Ioannis Kournoutas, Vera Vigo, Ricky Chae, Minghao Wang, Jose Gurrola, Adib A. Abla, Ivan El-Sayed, and Roberto Rodriguez Rubio. Acquisition of Volumetric Models of Skull Base Anatomy Using Endoscopic Endonasal Approaches: 3D Scanning of Deep Corridors Via Photogrammetry. World Neurosurgery, 129:372-377, sep 2019. 4

[35] Diana S. Jodeh, Heather Curtis, James J. Cray, Jonathan Ford, Summer Decker, and S. Alex Rottgers. Anthropometric Evaluation of Periorbital Region and Facial Projection Using Three-Dimensional Photogrammetry Journal of Craniofacial Surgery, 29[8]:2017-2020, nov 2018. 4

[36] Stanislao Grazioso, Mario Selvaggio, Teodorico Caporaso, and Giuseppe Di Gironimo. A Digital Photogrammetric Method to Enhance the Fabrication of Custom-Made Spinal Orthoses Journal of Prosthetics and Orthotics, 31[2]:133-139, apr 2019. 4

[37] Amaia Hernandez and Edward Lemaire. A smartphone photogrammetry method for digitizing prosthetic socket interiors. Prosthetics and orthotics international, pages $1-5,2017$. 4

[38] Rodrigo Salazar-Gamarra, Rosemary Seelaus, Jorge VIcente Lopes da Silva, Airton Moreira da Silva, and LuCIANO LAURIA Dib. Monoscopic photogrammetry to obtain 3D models by a mobile device: a method for making facial prostheses. Journal of otolaryngology - head \&S neck surgery $=$ Le Journal d'oto-rhinolaryngologie et de chirurgie cervico-faciale, 45[1]:33, may 2016. 426

[39] Steven B. Heymsfield, Brianna Bourgeois, Bennett K. NG, Markus J. Sommer, Xin Li, and John A. Shepherd. Digital anthropometry: A critical review. In European Journal of Clinical Nutrition, 72, pages 680-687. Nature Publishing Group, may 2018. 489

[40] TANIA S. DOUgLAS. Image processing for craniofacial landmark identification and measurement: A review of photogrammetry and cephalometry. Computerized Medical Imaging and Graphics, 28[7]:401-409, oct 2004. 4

[41] Kate Sutherland, Aidan J. Weichard, Margot J. Davey, Rosemary Sc Horne, Peter A. Cistulli, and Gillian M. NIXON. Craniofacial photography and association with sleep-disordered breathing severity in children. Sleep and Breathing, pages 1-7, aug 2019. 4.

[42] Ekaterina Sirazitdinova and Thomas M. Deserno. System Design for 3D Wound Imaging Using LowCost Mobile Devices In TeSSA S. COOK AND JiAnGUo Zhang, editors, Proc. SPIE 10138, Medical Imaging 2017: Imaging Informatics for Healthcare, Research, and Applications, 10138. International Society for Optics and Photonics, mar 2017. 4 
[43] Susanne Nahles, Martin Klein, Anke Yacoub, and Julia NEYER. Evaluation of positional plagiocephaly: Conventional anthropometric measurement versus laser scanning method Journal of CranioMaxillofacial Surgery, 46[1]:11-21, jan 2018. 476

[44] Taif Alawsi and Zainab Al-Bawi. A review of smartphone point-of-care adapter design. Engineering Reports, 1[2], sep 2019. 4

[45] G Fuchs and O Bayer. Eine radiologische Methode zur Bestimmung der Schädelkapazität. Radiol. Austriaca, 8:51-55, 1954. 10

[46] W BERgerhoff. Roentgenogram of basilar impression evaluated by measurement. Zentralblatt fur Neurochirurgie, 18[2-3]:149-162, 1957. 1031

[47] K Y Manjunath. Estimation Of Cranial Volumean Overview Of Methodologies. J Anat. Soc. India, 51[1]:85-91, 2002. 1018

[48] Z. Xu, T. H. Wu, Y. Shen, and L. Wue. Three dimentional reconstruction of large cultural heritage objects based on uav video and tls data. International Archives of the Photogrammetry, Remote Sensing and Spatial Information Sciences - ISPRS Archives, 41[July]:985-988, 2016. 13 [26

[49] Clive S. Fraser. Digital camera self-calibration $I S$ PRS Journal of Photogrammetry and Remote Sensing, 52[4]:149-159, aug 1997. 14,153945

[50] Sebahattin Bektas. Least square fitting of ellipsoid using orthogonal distances Boletim de Ciências Geodésicas, 21[2]:329-339, jun 2015. 1931

[51] Sebahattin Bektas. Orthogonal distance from an ellipsoid. Boletim de Ciências Geodésicas, 20[4]:970-983, 2014. 19

[52] Jan Falco Wilbrand, Alexander Szczukowski, Joerg Christoph Blecher, Joern Pons-Kuehnemann, Petros Christophis, Hans Peter Howaldt, and Heidrun SCHAAF. Objectification of cranial vault correction for craniosynostosis by three-dimensional photography Journal of Cranio-Maxillofacial Surgery, 40[8]:726-730, 2012. 22,23

[53] Gary B. Skolnick, Sybill D. Naidoo, Dennis C. Nguyen, Kamlesh B. Patel, and Albert S. Woo. Comparison of Direct and Digital Measures of Cranial Vault Asymmetry for Assessment of Plagiocephaly. The Journal of craniofacial surgery, 26[6]:1900-1903, 2015. 23

[54] P. Daponte, L. De Vito, F. Picariello, and M. Riccio. State of the art and future developments of measurement applications on smartphones Measurement, 46[9]:3291-3307, nov 2013. 26

[55] Krzysztof Bakula and Adrian Flasiński. Capabilities of a Smartphone for Georeferenced 3D Model Creation: An Evaluation In 13th International Multidisciplinary Scientific GeoConference SGEM 2013, 3, pages $16-22$, jun 2013. 26

[56] Amaia Hernandez and Edward Lemaire. A smartphone photogrammetry method for digitizing prosthetic socket interiors. Prosthetics and orthotics international, page 0309364616664150, sep 2016. 26, 41
[57] Albert K. Chong. Exploiting HD Camcorders for Close-Up Human Movement Applications The Photogrammetric Record, 27[138]:227-237, jun 2012. 26

[58] Jonathan T. Black and Richard S. Pappa. Videogrammetry Using Projected Circular Targets : Proofof-Concept Test In 21 st International Modal Analysis Conference, 2003. 26

[59] Shih-Yuan Lin, Jon P. Mills, and Peter D. Gosling. Videogrammetric monitoring of as-built membrane roof structures The Photogrammetric Record, 23[122]:128-147, jun 2008. 26

[60] Karl Kraus. Photogrammetry: Advanced Methods and Applications/by Karl Kraus. Dümmler, 1997. 28,3349

[61] Inés Barbero-García, José Luis Lerma, Ángel MarquésMateu, and Pablo Miranda. Low-Cost SmartphoneBased Photogrammetry for the Analysis of Cranial Deformation in Infants World Neurosurgery,

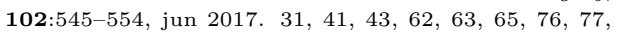
95100

[62] Jonathan Pindrik, Joseph Molenda, Rafael UribeCardenas, Amir h Dorafshar, and Edward S. Ahn. Normative Ranges of Anthropometric Cranial Indices and Metopic Suture Closure during Infancy. Journal of Neurosurgery: Pediatrics, 18[December]:667-673, 2016. 31

[63] Clive S. Fraser. Photogrammetric measurement to one part in a million Photogrammetric Engineering and Remote Sensing, 58[3]:305-310, 1992. 33

[64] Clive S. Fraser. Network design. In K. B. AtKinSON, editor, Close-Range photogrammetry and Machine Vision, pages 256-281. Whittles, Caithness, Scotland, 1996. 33

[65] José Luis Lerma. Fotogrametría moderna: analítica y digital. Universidad Politecnica de Valencia, 2002. 33

[66] Chin-Heng Wang, Jon P Mills, and Pauline E Miller. Automated Low-Cost Photogrammetry for Flexible Structure Monitoring. International Archives of the Photogrammetry, Remote Sensing and Spatial Information Sciences, 39[September]:393-398, 2012. 41

[67] Natan Micheletti, Jim H. Chandler, and Stuart N. Lane. Investigating the geomorphological potential of freely available and accessible structure-frommotion photogrammetry using a smartphone Earth Surface Processes and Landforms, 40[4]:473-486, mar 2015. 41

[68] E Nocerino, F Lago, D Morabito, F Remondino, L Porzi, F Poiesi, S. Rota Bulo, P Chippendale, A Locher, M Havlena, L. Van Gool, M Eder, A Fötschl, A Hilsmann, L KAUSCH, AND P EISERT. A smartphone-based 3D pipeline for the creative industry - The replicate eu project In International Archives of the Photogrammetry, Remote Sensing and Spatial Information Sciences - ISPRS Archives, 42, pages 535-541, 2017. 41

[69] Mauren Abreu de Souza, Stuart Robson, and Jeremy C. Hebden. A Photogrammetric Technique for Acquiring Accurate Head Surfaces of Newborn Infants for Optical Tomography Under Clinical Conditions The Photogrammetric Record, 27[139]:253-271, sep 2012. 41 . 
70] O. Hellwich, A. Rose, T. Bien, C. Malolepszy, D. Mucha, AND T. KRÜGer. Patient Registration using Photogrammetric Surface Reconstruction for Smartphone Imagery ISPRS - International Archives of the Photogrammetry, Remote Sensing and Spatial Information Sciences, XLI-B5:829-833, jun 2016. 41

[71] Albert K. Chong and Gretchen Brownstein. A technique for improving webcam precision in biological plant measurement Photogrammetric Record, 25[130]:180-196, sep 2010. 4161

[72] Clive S. Fraser. Automatic camera calibration in close range photogrammetry. Photogrammetric Engineering and Remote Sensing, 79[4], 2013. 42

73] Devrim AkCa and Armin Gruen. Comparative geometric and radiometric evaluation of mobile phone and still video cameras The Photogrammetric Record, 24[127]:217-245, sep 2009. 42

[74] Fabio Remondino, Maria Grazia Spera, Erica Nocerino, Fabio Menna, and Francesco Nex. State of the art in high density image matching The Photogrammetric Record, 29[146]:144-166, jun 2014. 42

[75] Fabio Remondino, Silvio Del Pizzo, Thomas P. Kersten, AND SAlvatore Troisi. Low-cost and open-source solutions for automated image orientation- A critical overview Lecture Notes in Computer Science, 7616:40-54, 2012. 42

[76] David G Lowe. Object Recognition from Local Scale-Invariant Features In Proceedings of the International Conference on Computer Vision-Volume 2 Volume 2, ICCV '99, pages 1150--, Washington, DC, USA, 1999. IEEE Computer Society. 42,82

[77] Herbert Bay, Andreas Ess, Tinne Tuytelaars, and LuC VAN Gool. Speeded-Up Robust Features (SURF) 2007. 4282

[78] Clive S. Fraser, Alex Woods, and Danny Brizzi. Hyper Redundancy for Accuracy Enhancement in Automated Close Range Photogrammetry The Photogrammetric Record, 20[111]:205-217, sep 2005. 42

[79] B Alsadik, M Gerke, and G Vosselman. Efficient Use of Video for 3D Modelling of Cultural Heritage Objects ISPRS Annals of Photogrammetry, Remote Sensing and Spatial Information Sciences, II-3/W4:1-8, 2015. 42

[80] Fabio Remondino and Clive S Fraser. Digital camera calibration methods: considerations and comparisons In ISPRS Commission V Symposium 'Image Engineering and Vision Metrology', WG V/I, pages 266-272, 2006. 4345

[81] Clive S. Fraser. Innovations in automation for vision metrology systems The Photogrammetric Record, 15[90]:901-911, oct 1997. 43

[82] Thomas Luhmann, Clive S. Fraser, and Hans-Gerd Maas. Sensor modelling and camera calibration for \begin{tabular}{ll|l|}
\hline close-range photogrammetry & 2016.43 \\
\hline
\end{tabular}

[83] José L. Lerma, Santiago Navarro, Miriam Cabrelles, and Ana E. Seguí. Camera Calibration with Baseline Distance Constraints The Photogrammetric Record, 25[130]:140-158, jun 2010. 44
[84] José Luis Lerma, Santiago Navarro, Ana E. Seguí, and Miriam Cabrelles. Range-based Versus Automated Markerless Image-Based Techniques for Rock Art Documentation. The Photogrammetric Record, 29[145]:30-48, 2014. 44

[85] J. G. Fryer. Camera calibration. In K. B. Atkinson, editor, Close Range Photogrammetry and Machine Vision, chapter 6 , pages 56-79. Whittles, Caithness, Scotland, 2001. 45

[86] Clive S. Fraser and Kenneth L Edmundson. Design and implementation of a computational processing system for off-line digital close-range photogrammetry ISPRS Journal of Photogrammetry and Remote Sensing, 55[2]:94-104, jun 2000. 49

[87] Pierre Drap and Julien Lefèvre. An Exact Formula for Calculating Inverse Radial Lens Distortions Sensors, 16[6]:807, jun 2016. 49

[88] Thomas Luhmann and Klaus Wendt. Recommendations for an Acceptance and Verification Test of Optical 3-D Measurement Systems International Archives of Photogrammetry and Remote Sensing, XXXIII[Part B5]:493-500, 2000. 50

[89] H. Schaaf, J. Pons-Kuehnemann, C. Y. Malik, P. Streckbein, M. Preuss, H. P. Howaldt, and J. F. Wilbrand. Accuracy of three-dimensional photogrammetric images in non-synostotic cranial deformities. Neuropediatrics, 41[1]:24-29, 2010. 5967

[90] Derick A. Mendonca, Sybill D. Naidoo, Gary Skolnick, Rachel Skladman, and Albert S. Woo. Comparative study of cranial anthropometric measurement by traditional calipers to computed tomography and three-dimensional photogrammetry. Journal of Craniofacial Surgery, 24[4]:1106-1110, 2013. 61 67 73

[91] Olivia A Ho, Nikoo Saber, Derek Stephens, April Clausen, James Drake, Christopher Forrest, and John Phillips. Comparing the Use of 3D Photogrammetry and Computed Tomography in Assessing the Severity of Single-Suture Nonsyndromic Craniosynostosis. Plastic Surgery, 25[2]:78-83, 2017. 61

[92] Tasha E Metzger, Katherine S Kula, George J Eckert, And Ahmed A. Ghoneima. Orthodontic soft-tissue parameters: A comparison of cone-beam computed tomography and the 3dMD imaging system American Journal of Orthodontics and Dentofacial Orthopedics, 144[5]:672-681, 2013. 61.

[93] Sören Kottner, Lars C. Ebert, Garyfalia Ampanozi, Marcel Braun, Michael J. Thali, and Dominic Gascho. VirtoScan - a mobile, low-cost photogrammetry setup for fast post-mortem 3D full-body documentations in x-ray computed tomography and autopsy suites Forensic Science, Medicine, and Pathology, 13[1]:34-43, mar 2017. 616273

[94] Caroline A.A. Beaumont, Paul G.M. Knoops, Alessandro Borghi, N.U. Owase Jeelani, MaArten J Koudstaal, Silvia Schievano, David J Dunaway, and Naiara RODRIGUEZ-FLOREZ. Three-dimensional surface scanners compared with standard anthropometric measurements for head shape Journal of CranioMaxillofacial Surgery, 45[6]:921-927, jun 2017. 61]73 
[95] Douglas R. McKay, Kristen M. Davidge, Susanne K. Williams, Lloyd A. Ellis, David K. Chong, Rodrigo P. Teixeira, Andrew L. Greensmith, and Anthony D. HOLMES. Measuring cranial vault volume with three-dimensional photography: A method of measurement comparable to the gold standard. Journal of Craniofacial Surgery, 21[5]:1419-1422, 2010. 62.

[96] Gary B. Skolnick, Sybill D. Naidoo, Dennis C. Nguyen, Kamlesh B. Patel, and Albert S. Woo. Comparison of Direct and Digital Measures of Cranial Vault Asymmetry for Assessment of Plagiocephaly. The Journal of craniofacial surgery, 26[6]:1900-1903, 2015. 6273

[97] José Luis Lerma, Inés Barbero-García, Ángel MarquésMateu, and Pablo Miranda. Smartphone-based video for 3D modelling: Application to infant's cranial deformation analysis Measurement, 116:299306, 2018. 62 66 101

[98] Inés Barbero-García, Miriam Cabrelles, José Luis Lerma, AND ÁNGel MARqués-Mateu. Smartphone-based closerange photogrammetric assessment of spherical objects The Photogrammetric Record, 33[162]:283299, jun 2018. 65] $678285,95[101$

[99] John Persing, Hector James, Jack Swanson, and John Kattwinkel. Prevention and Management of Positional Skull Deformities in Infants. American Academy of Pediatrics, 112[1]:199-202, 2003. 74

[100] Nicette Sergueef, Kenneth E. Nelson, and Thomas GLONEK. Palpatory diagnosis of plagiocephaly Complementary Therapies in Clinical Practice, 12[2]:101-110, may 2006. 74

[101] Matthias Kreutz, Brigitte Fitze, Christoph Blecher, Augello Marcello, Ruben Simon, Rebecca Cremer, HansFlorian Zeilhofer, Christoph Kunz, and Johannes Mayr. Facial asymmetry correction with moulded helmet therapy in infants with deformational skull base plagiocephaly. Journal of cranio-maxillo-facial surgery : official publication of the European Association for Cranio-Maxillo-Facial Surgery, 46[1]:28-34, jan 2018. 74

[102] Inés Barbero-García, José Luis Lerma, Pablo MIRANDA, AND ÁNGEL MARqUÉS-MATEU. Smartphone-based photogrammetric 3D modelling assessment by comparison with radiological medical imaging for cranial deformation analysis Measurement, 131:372-379, jan 2019. 76 82 101

[103] G. A. De Jong, T. J.J. Maal, and H. Delye. The computed cranial focal point Journal of CranioMaxillofacial Surgery, 43[9]:1737-1742, nov 2015. 78 97

[104] E Nocerino, F Poiesi, A Locher, Y T Tefera, F Remondino, P Chippendale, and L. Van Gool. 3D Reconstruction with a Collaborative Approach Based on Smartphones and a Cloud-based Server IS PRS - International Archives of the Photogrammetry, Remote Sensing and Spatial Information Sciences, XLII2/W8[November]:187-194, nov 2017. 81
105] Chengyou Wang, Yunpeng Zhang, and Xiao Zhou. Robust Image Watermarking Algorithm Based on ASIFT against Geometric Attacks Applied Sciences, 8[3]:410, mar 2018. 82

106] Rafael Muñoz-Salinas, Manuel J Marín-Jimenez, Enrique Yeguas-Bolivar, And R Medina-CARnicer. Mapping and Localization from Planar Markers Pattern Recognition, 73:158-171, 2018. 82

[107] S Garrido-Jurado, R Muñoz-Salinas, F J Madrid-Cuevas, AND $\mathrm{R}$ MEDINA-CARNICER. Generation of fiducial marker dictionaries using mixed integer linear programming Pattern Recognition, 51:481-491, 2016. 82

108] Francisco J. Romero-Ramirez, Rafael Muñoz-Salinas, and Rafael Medina-Carnicer. Speeded up detection of squared fiducial markers Image and Vision Computing, 76:38-47, aug 2018. 82

[109] Sérgio Leandro Alves Da Silva, Antonio Maria Garcia Tommaselli, and Almir Olivette Artero. Utilização de alvos codificados do tipo ARUCO na automação do processo de calibração de câmaras Boletim de Ciencias Geodesicas, 20[3]:636-656, sep 2014. 82

110] M. Pierrot Deseilligny and I Clery. Apero, an Open Source Bundle Adjusment Software for Automatic Calibration and Orientation of Set of Images ISPRS - International Archives of the Photogrammetry, Remote Sensing and Spatial Information Sciences, XXXVIII-5/[snavely]:269-276, 2012. 85

[111] Paolo Cignoni, Marco Callieri, Massimiliano Corsini, matteo Dellepiane, Fabio Ganovelli, and Guido Ranzuglia. MeshLab: an Open-Source Mesh Processing Tool. In VitTorio Scarano, Rosario De ChIARA, AND UGo ERRA, editors, Eurographics Italian Chapter Conference. The Eurographics Association, 2008. 87

[112] Fausto Bernardini, Joshua Mittleman, Holly Rushmeier, Claudio Silva, and Gabriel Taubin. The ball-pivoting algorithm for surface reconstruction. IEEE Transactions on Visualization and Computer Graphics, 5[4]:349-359, 1999. 87

[113] Paul J. Besl and Neil D. McKay. Method for registation of 3-D shapes In PAUL S. SCHENKER, editor, Sensor Fusion IV: Control Paradigms and Data Structures, 1611, pages 586-606. SPIE, apr 1992. 89.

[114] S Goebbels, R Pohle-Fröhlich, AND P Pricken. Iterative Closest Point Algorithm for Accurate Registration of Coarsely Registered Point Clouds with CityGML Models In ISPRS Annals of the Photogrammetry, Remote Sensing and Spatial Information Sciences, 4, pages 201-208, 2019. 89

[115] Bruno Pereira Silva, Emilio Jiménez-Castellanos, Rafael Martinez-de Fuentes, Ana Aida Vilches Fernandez, and Stephen Chu. Perception of maxillary dental midline shift in asymmetric faces. The international journal of esthetic dentistry, 10[4]:588-596, 2015. 95 101 
[116] L. Camison, M. Bykowski, W. W. Lee, J. C. Carlson, J. Roosenboom, J. A. Goldstein, J. E. Losee, and S. M. WeINBERG. Validation of the Vectra $\mathrm{H} 1$ portable three-dimensional photogrammetry system for facial imaging. International Journal of Oral and Maxillofacial Surgery, 47[3]:403-410, mar 2018. 98

[117] Eduardo Joaquim lopes Alho, Carlo Rondinoni Fabio OKuda FurokaWa, and Bernardo A. Monaco, Computer-assisted craniometric evaluation for diagnosis and follow-up of craniofacial asymmetries: SymMetric v. 1.0. Child's Nervous System, pages $1-7$, dec 2019. 98

[118] Kristina Aldridge, Simeon A. Boyadjiev, George T. Capone, Valerie B. Deleon, and Joan T. Richtsmeier.
Precision and error of three-dimensional phenotypic measures acquired from 3dMD photogrammetric images. American Journal of Medical Genetics, 138 A [3]:247-253, 2005. 98

[119] Inés Barbero-García and José Luis Lerma. Assessment of Registration Methods for Cranial 3D Modelling In Proceedings, 19, 2019. 101

[120] InÉs Barbero-García, José Luis Lerma, and Gaspar MoraNAVARRo. Fully automatic smartphone-based photogrammetric 3D modelling of infant's heads for cranial deformation analysis. ISPRS Journal of Photogrammetry and Remote Sensing, 166:268-277, 2020. 101,102 